\title{
A Diastereoselective Radical Cyclization Approach to
}

\section{Substituted Quinuclidines}

\author{
Thomas A. Hunt,,$^{\dagger}$ Andrew F. Parsons ${ }^{* \dagger}$ and Robert Pratt
}

Department of Chemistry, University of York, Heslington, York, YO10 5DD, UK and Vernalis Research Ltd, Oakdene Court, 613 Reading Road, Winnersh, Wokingham, RG41 5UA, UK

afp2@york.ac.uk

$\dagger$ University of York

$\ddagger$ Vernalis Research Ltd

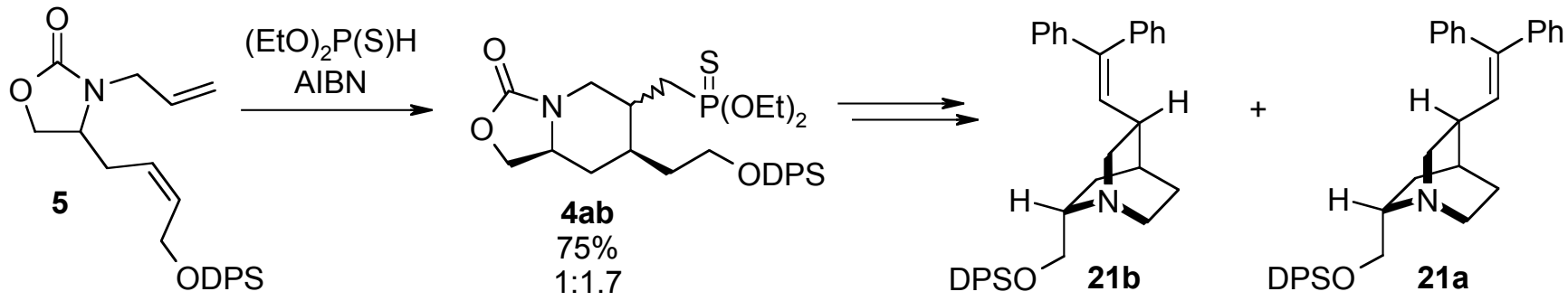

\section{Contents}

General Experimental

S2

Experimental Procedures, ${ }^{1} \mathrm{H}$ and ${ }^{13} \mathrm{C}$ NMR Spectra for Compounds 4,5,7-10, 16-20

S3-S31

Full NMR Assignment and 1D and 2D NMR Spectra for Compounds 21a and 21b

S32-S39

Experimental Procedure, ${ }^{1} \mathrm{H}$ and ${ }^{13} \mathrm{C}$ NMR Spectra for Compound 22b

S39-S45 


\section{General Experimental}

${ }^{1} \mathrm{H}$ and ${ }^{13} \mathrm{C}$ NMR were recorded on a $400 \mathrm{MHz}$ spectrometer at ambient temperature. Chemical shifts are given in parts per million ( $\mathrm{ppm}$ ) referenced to the $\mathrm{CDCl}_{3}$ residual solvent peak measured downfield from TMS which was used as an internal standard. ${ }^{13} \mathrm{C}$ NMR spectra are recorded with broad band proton decoupling and assignments were made using DEPT experiments. ${ }^{1} \mathrm{H}$ NMR assignments were aided by ${ }^{1} \mathrm{H}-{ }^{1} \mathrm{H}$ COSY, ${ }^{1} \mathrm{H}-{ }^{13} \mathrm{C}$ HSQC and NOESY experiments where necessary. Infra-red spectra were recorded either as solutions in $\mathrm{CDCl}_{3}$ or $\mathrm{CH}_{2} \mathrm{Cl}_{2}$ or as films between $\mathrm{NaCl}$ plates. Absorption maximum $\left(v_{\max }\right)$ are reported in wavenumbers $\left(\mathrm{cm}^{-1}\right)$ and only selected peaks are noted. Thin layer chromatography (TLC) was performed on aluminium backed silica gel plates and visualised with a UV lamp (254 nm or $355 \mathrm{~nm}$ ), or in an aqueous solution of $\mathrm{KMnO}_{4} . \mathrm{R}_{\mathrm{f}}$ values are given to the nearest 0.1. Organic extracts were dried over anhydrous magnesium sulfate. Flash column chromatography was performed using flash silica $60,32-63 .{ }^{n} \mathrm{BuLi}$ and ${ }^{\mathrm{s}} \mathrm{BuLi}$ were titrated before use against $N$ benzylbenzamide. Petroleum ether refers to the fraction of boiling range $40-60{ }^{\circ} \mathrm{C}$. Solvents were purified/dried using standard literature procedures. 


\section{tert-Butyl[(3-iodo-2-propynyl)oxy]diphenylsilane 7}

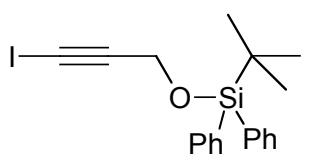

To a solution of tert-butyl(diphenyl)(2-propynyloxy)silane (10.0 g, $34.0 \mathrm{mmol})$ in anhydrous THF (200 $\mathrm{mL})$ at $-78{ }^{\circ} \mathrm{C}$ was added dropwise ${ }^{\mathrm{n}} \mathrm{BuLi}(15.0 \mathrm{~mL}$ of a $2.5 \mathrm{M}$ solution in hexanes, $37.4 \mathrm{mmol})$. The reaction mixture was stirred at $-78^{\circ} \mathrm{C}$ for $1 \mathrm{~h}$ after which time a solution of iodine $(10.4 \mathrm{~g}, 40.8 \mathrm{mmol})$ in anhydrous THF (100 mL) was added and the reaction was warmed to $\mathrm{rt}$ over $1 \mathrm{~h}$. The reaction mixture was diluted with $\mathrm{Et}_{2} \mathrm{O}(500 \mathrm{~mL})$, then washed with $\mathrm{Na}_{2} \mathrm{~S}_{2} \mathrm{O}_{3}$ (saturated aqueous solution) and then once with brine. The organic phase was dried $\left(\mathrm{MgSO}_{4}\right)$, filtered and evaporated to give a brown oil. Purification by flash column chromatography through a silica plug using petroleum ether/EtOAc (9:1) as eluent afforded alkynyl iodide $7(13.7 \mathrm{~g}, 96 \%)$ as a yellow oil. $\mathrm{R}_{\mathrm{f}} 0.5$ (petroleum ether); IR: (film) $v_{\max } 3071,2959,2931,2858,2192,1472,1427,1371,1113,1087,997 \mathrm{~cm}^{-1} ;{ }^{1} \mathrm{H} \mathrm{NMR}(400 \mathrm{MHz}$, $\left.\mathrm{CDCl}_{3}\right) \delta_{\mathrm{H}}: 7.70-7.68(\mathrm{~m}, 4 \mathrm{H}), 7.46-7.37(\mathrm{~m}, 6 \mathrm{H}), 4.45(\mathrm{~s}, 2 \mathrm{H}), 1.06(\mathrm{~s}, 9 \mathrm{H}) ;{ }^{13} \mathrm{C} \mathrm{NMR}(100 \mathrm{MHz}$, $\left.\mathrm{CDCl}_{3}\right) \delta_{\mathrm{C}}: 135.6(\mathrm{CH}), 132.8(\mathrm{C}), 129.8(\mathrm{CH}), 127.7(\mathrm{CH}), 92.6(\mathrm{C}), 54.1\left(\mathrm{CH}_{2}\right), 26.7\left(\mathrm{CH}_{3}\right), 14.2(\mathrm{C})$, 1.57 (C); LRMS m/z $438\left(\left[\mathrm{M}+\mathrm{NH}_{4}\right]^{+}, 7 \%\right), 421$ (1) 380 (13), 360 (45), 196 (100); HRMS (CI, NH $)_{3 / z}$ $\left[\mathrm{M}+\mathrm{NH}_{4}\right]^{+}$found 438.1749; calcd 438.0750 for $\mathrm{C}_{19} \mathrm{H}_{24} \mathrm{IOSi}$. 


\section{Methyl 2-[(tert-butoxycarbonyl)amino]-6-\{[tert-butyl(diphenyl)silyl]oxy\}-4-hexynoate 8}

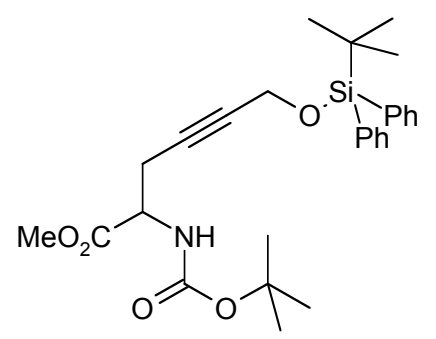

Anhydrous THF was degassed by a freeze-pump-thaw process repeated three times. A round-bottomed flask was charged with zinc dust $(7.96 \mathrm{~g}, 122 \mathrm{mmol})$ and flushed with nitrogen. To this was added anhydrous THF $(80 \mathrm{~mL})$ and 1,2-dibromoethane $(0.52 \mathrm{~mL}, 6.08 \mathrm{mmol})$. The reaction mixture was stirred and, with a hot-air gun, brought to reflux then cooled back to rt, this cycle was repeated three times. Freshly distilled TMSCl $(0.48 \mathrm{~mL}, 3.78 \mathrm{mmol})$ was added and the reaction was stirred at $\mathrm{rt}$ for $0.1 \mathrm{~h}$. A solution of iodide $6(20.0 \mathrm{~g}, 60.8 \mathrm{mmol})$ in anhydrous THF $(20 \mathrm{~mL})$ was added at $\mathrm{rt}$. The reaction was stirred at $\mathrm{rt}$ for $0.75 \mathrm{~h}$ (the reaction was observed to be slightly exothermic reaching $c a .30$ ${ }^{\circ} \mathrm{C}$, cooling was induced by placing into an ice bath) after which time stirring was ceased to allow excess zinc to settle (further reaction was retarded by cooling the reaction vessel in a dry ice bath). Meanwhile, a round bottomed flask, equipped with a stirrer bar, was charged with lithium chloride (5.04 g, 122 mmol), which was subsequently dried under vacuum at $130{ }^{\circ} \mathrm{C}$ for $4 \mathrm{~h}$. To this flask was added $\mathrm{CuCN}$ (5.44 g, $60.8 \mathrm{mmol})$ and anhydrous THF (40 mL), the mixture was stirred at $\mathrm{rt}$ until a yellow solution was obtained that was then cooled to $-10{ }^{\circ} \mathrm{C}$. The previously prepared solution of the organozinc iodide was decanted from the excess zinc via syringe and added dropwise to the solution of the copper complex. The reaction mixture was stirred at $-10{ }^{\circ} \mathrm{C}$ for $0.2 \mathrm{~h}$ then cooled to $-78{ }^{\circ} \mathrm{C}$ and a solution of iodoalkyne 7 (20.4 g, $48.8 \mathrm{mmol})$ in anhydrous THF $(20 \mathrm{~mL})$ was added. The reaction was stirred at $20{ }^{\circ} \mathrm{C}$ for $4 \mathrm{~h}$ and then at $\mathrm{rt}$ for $16 \mathrm{~h}$. $\mathrm{NH}_{4} \mathrm{Cl}$ (saturated aqueous solution) was added and then extracted with EtOAc, the combined organic phases were dried $\left(\mathrm{MgSO}_{4}\right)$, filtered and evaporated to an orange residue. Purification by flash column chromatography (on silica) using petroleum ether/ $\operatorname{Et}_{2} \mathrm{O}$ (9:1 to 4:1) as eluent afforded alkyne $\mathbf{8}\left(16.5 \mathrm{~g}, 68 \%\right.$ ) as a colorless oil. $\mathrm{R}_{\mathrm{f}} 0.8$ (petroleum ether:Et ${ }_{2} \mathrm{O}$ 4:1); IR: 
$\left(\mathrm{CH}_{2} \mathrm{Cl}_{2}\right) v_{\max }\left(\mathrm{CH}_{2} \mathrm{Cl}_{2}\right) 3433,2931,2860,1747,1714,1503,1367,1168,1112 \mathrm{~cm}^{-1} ;{ }^{1} \mathrm{H}$ NMR $(400$ $\left.\mathrm{MHz}, \mathrm{CDCl}_{3}\right) \delta_{\mathrm{H}}: 7.71-7.69(\mathrm{~m}, 4 \mathrm{H}), 7.46-7.38(\mathrm{~m}, 6 \mathrm{H}), 5.27(\mathrm{~d}, J=8.5 \mathrm{~Hz}, 1 \mathrm{H}), 4.43(\mathrm{dt}, J=8.5,5.0$ $\mathrm{Hz}, 1 \mathrm{H}), 4.28(\mathrm{t}, J=2.0 \mathrm{~Hz}, 2 \mathrm{H}), 3.73(\mathrm{~s}, 3 \mathrm{H}), 2.81-2.64(\mathrm{~m}, 2 \mathrm{H}), 1.46(\mathrm{~s}, 9 \mathrm{H}), 1.05(\mathrm{~s}, 9 \mathrm{H}) ;{ }^{13} \mathrm{C} \mathrm{NMR}$ $\left(100 \mathrm{MHz}, \mathrm{CDCl}_{3}\right) \delta_{\mathrm{C}}: 171.1(\mathrm{C}), 155.0(\mathrm{C}), 135.5(\mathrm{CH}), 133.0(\mathrm{C}), 129.7(\mathrm{CH}), 127.6(\mathrm{CH}), 81.7(\mathrm{C})$, $80.0(\mathrm{C}), 79.4(\mathrm{C}), 52.6\left(\mathrm{CH}_{2}\right), 52.4\left(\mathrm{CH}_{3}\right), 51.9(\mathrm{CH}), 28.2\left(\mathrm{CH}_{3}\right), 26.6\left(\mathrm{CH}_{3}\right), 23.0\left(\mathrm{CH}_{2}\right), 19.0(\mathrm{C})$; LRMS (CI, $\left.\mathrm{NH}_{3}\right)$ m/z $396\left([\mathrm{M}-\mathrm{Boc}] \mathrm{H}^{+}, 100 \%\right)$; HRMS (CI, $\left.\mathrm{NH}_{3}\right) m / z[\mathrm{M}-\mathrm{Boc}] \mathrm{H}^{+}$found 396.2000; calcd 396.1995 for $\mathrm{C}_{23} \mathrm{H}_{29} \mathrm{NO}_{3} \mathrm{Si}$.

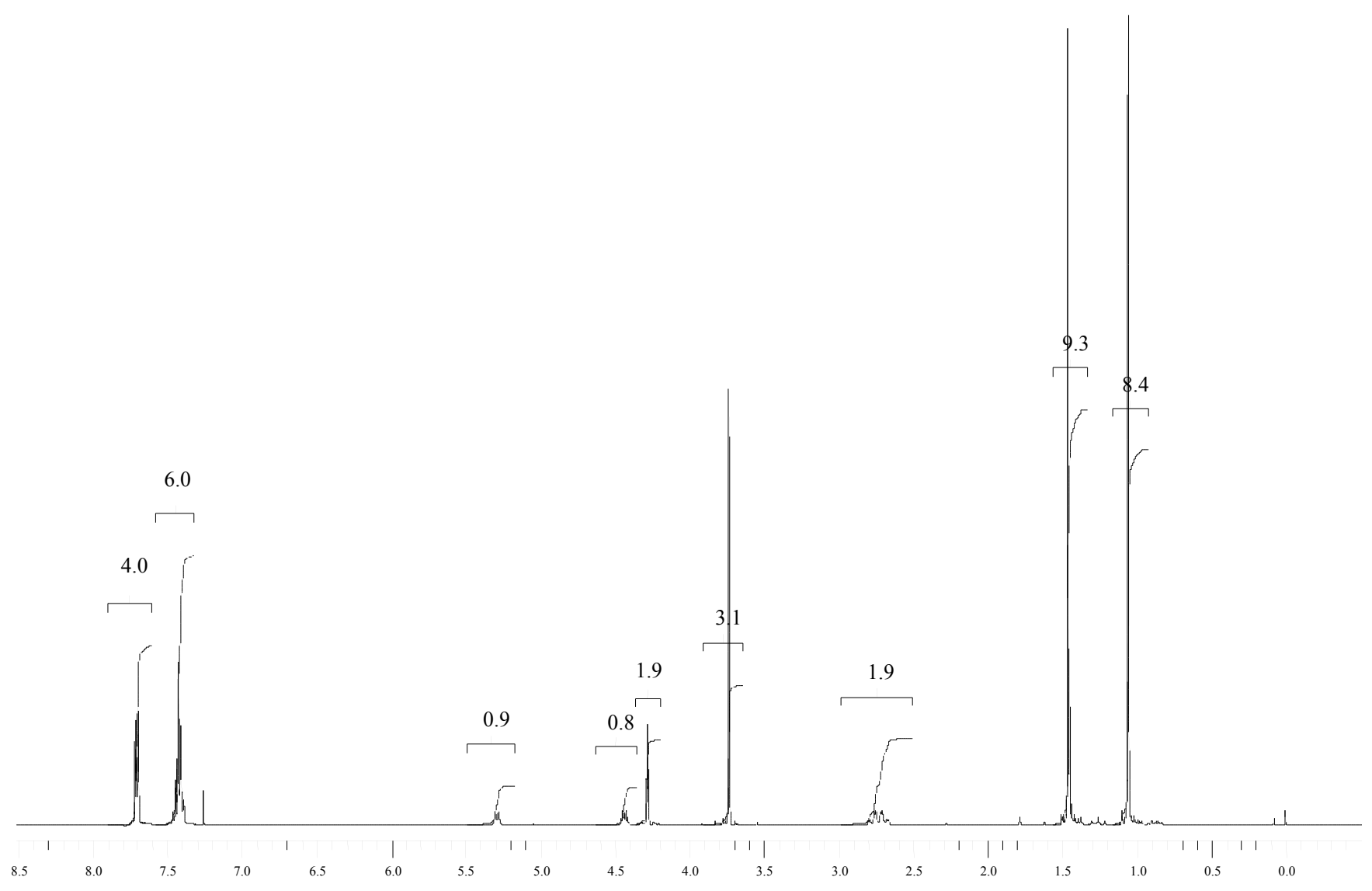



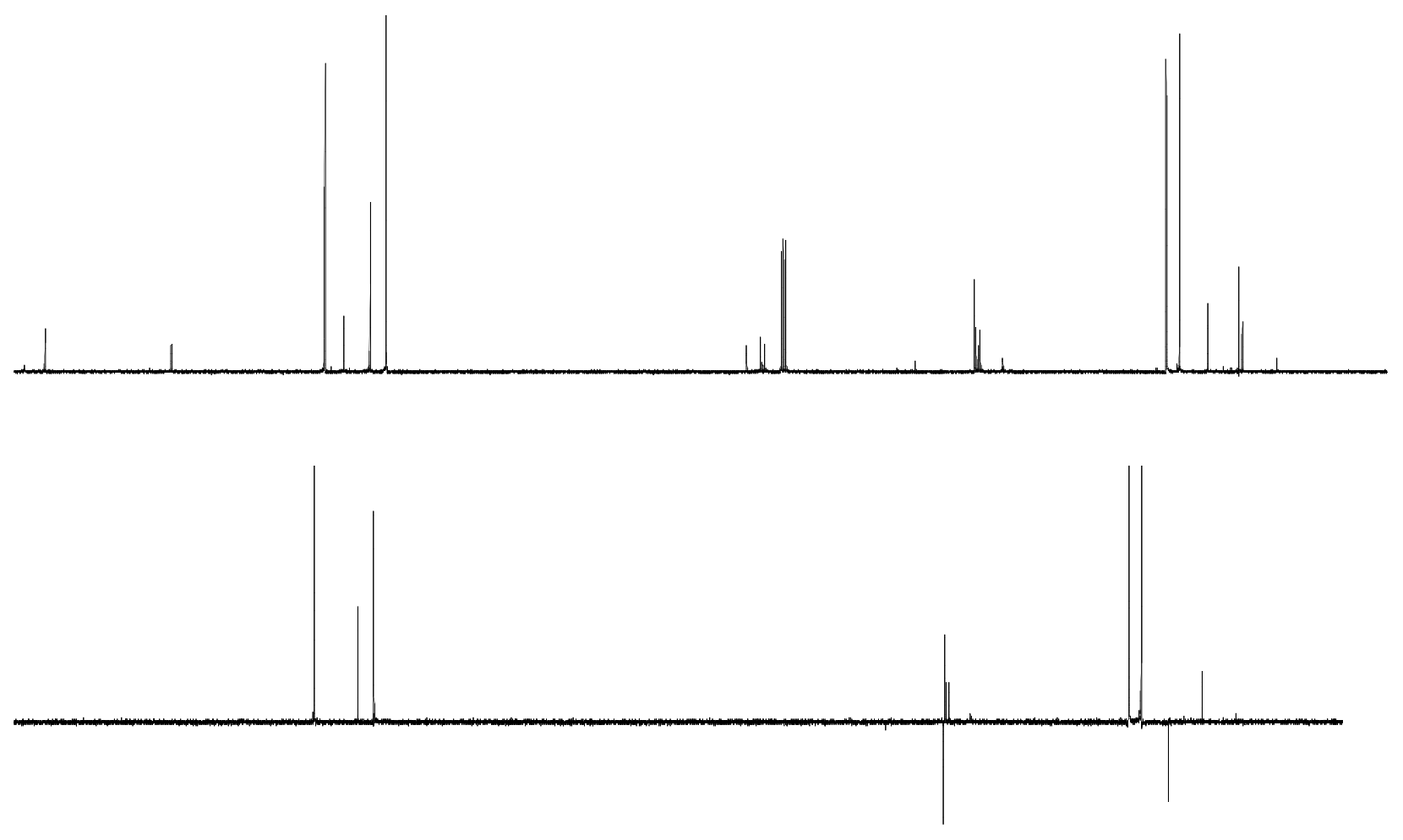

ppm

Methyl (Z)-2-[(tert-butoxycarbonyl)amino]-6-\{[tert-butyl(diphenyl)silyl]oxy\}-4-hexenoate 9

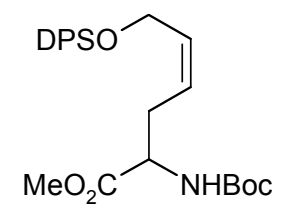

Alkyne 8 (14.5 g, $29.3 \mathrm{mmol})$ was dissolved in petroleum ether/EtOAc $(1: 1,500 \mathrm{~mL})$, the solution was subsequently degassed by bubbling a continuous stream of nitrogen through the solution for $0.1 \mathrm{~h}$. Rosenmund's catalyst $\left(\mathrm{Pd} / \mathrm{BaSO}_{4}, 5 \% \mathrm{w} / \mathrm{w}, 3.80 \mathrm{~g}\right)$ was added and the reaction was left stirring under a $\mathrm{H}_{2}$ atmosphere for $10 \mathrm{~h}$. After this time the catalyst was removed by filtration (Celite), and the filtrate was concentrated in vacuo. The crude residue was purified by flash column chromatography (on silica) using petroleum ether/EtOAc (2:1) as eluent to afford alkene $\mathbf{9}(13.3 \mathrm{~g}, 90 \%)$ as a colorless oil. From the ${ }^{1} \mathrm{H}$ NMR spectrum the purity of alkene 9 was estimated to be approximately $70 \%$ containing $30 \%$ of alkane 11 (by comparison of the integrals values of the signals between 7.68-7.38 $(10 \mathrm{H})$ and 2.47-2.26 (2H) ppm). $\mathrm{R}_{\mathrm{f}} 0.7$ (petroleum ether:EtOAc 2:1); IR: $\left(\mathrm{CH}_{2} \mathrm{Cl}_{2}\right) v_{\max } 3439,3369,2955,2931,2857,1746$, 
$1717,1501,1428,1365,1169,1112 \mathrm{~cm}^{-1} ;{ }^{1} \mathrm{H}$ NMR $\left(400 \mathrm{MHz}, \mathrm{CDCl}_{3}\right) \delta_{\mathrm{H}}: 7.68-7.64(\mathrm{~m}, 4 \mathrm{H}), 7.45-7.38$ (m, 6H), 5.79-5.73 (m, 1H), 5.37-5.30 (m, 1H), $5.00(\mathrm{br} \mathrm{s}, 1 \mathrm{H}), 4.34-4.25(\mathrm{~m}, 1 \mathrm{H}), 4.19(\mathrm{~d}, J=6.0 \mathrm{~Hz}$, 2H), $3.72(\mathrm{~s}, 3 \mathrm{H}), 2.47-2.38(\mathrm{~m}, 1 \mathrm{H}), 2.37-2.26(\mathrm{~m}, 1 \mathrm{H}), 1.44(\mathrm{~s}, 9 \mathrm{H}), 1.04(\mathrm{~s}, 9 \mathrm{H}) ;{ }^{13} \mathrm{C}$ NMR $(100 \mathrm{MHz}$, $\left.\mathrm{CDCl}_{3}\right) \delta_{\mathrm{C}}: 172.4(\mathrm{C}), 155.1(\mathrm{C}), 135.4(\mathrm{CH}), 133.3(\mathrm{CH}), 133.0(\mathrm{C}), 129.6(\mathrm{CH}), 127.6(\mathrm{CH}), 124.2$ (CH), $79.7(\mathrm{C}), 59.9\left(\mathrm{CH}_{2}\right), 52.8\left(\mathrm{CH}_{3}\right), 52.1(\mathrm{CH}), 30.2\left(\mathrm{CH}_{2}\right), 28.2\left(\mathrm{CH}_{3}\right), 26.7\left(\mathrm{CH}_{3}\right), 19.0(\mathrm{C})$; LRMS $\left(\mathrm{CI}, \mathrm{NH}_{3}\right) m / z 498\left(\mathrm{M}+\mathrm{H}^{+}, 5 \%\right), 398\left(100,[\mathrm{M}-\mathrm{Boc}] \mathrm{H}^{+}\right)$; HRMS (CI, $\left.\mathrm{NH}_{3}\right) \mathrm{m} / z[\mathrm{M}+\mathrm{H}]^{+}$found 498.2678; calcd 498.2676 for $\mathrm{C}_{28} \mathrm{H}_{39} \mathrm{NO}_{5} \mathrm{Si}$.

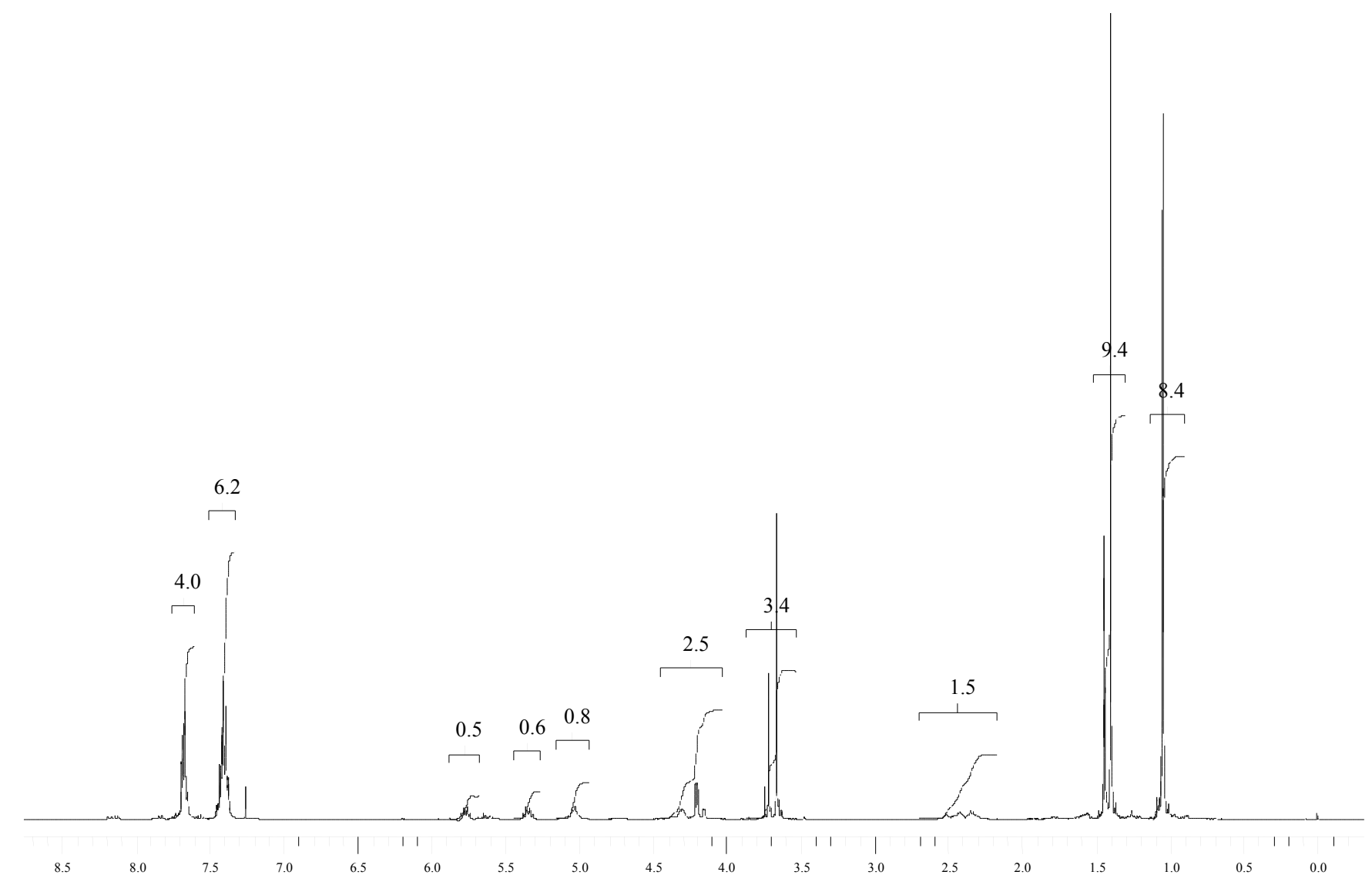



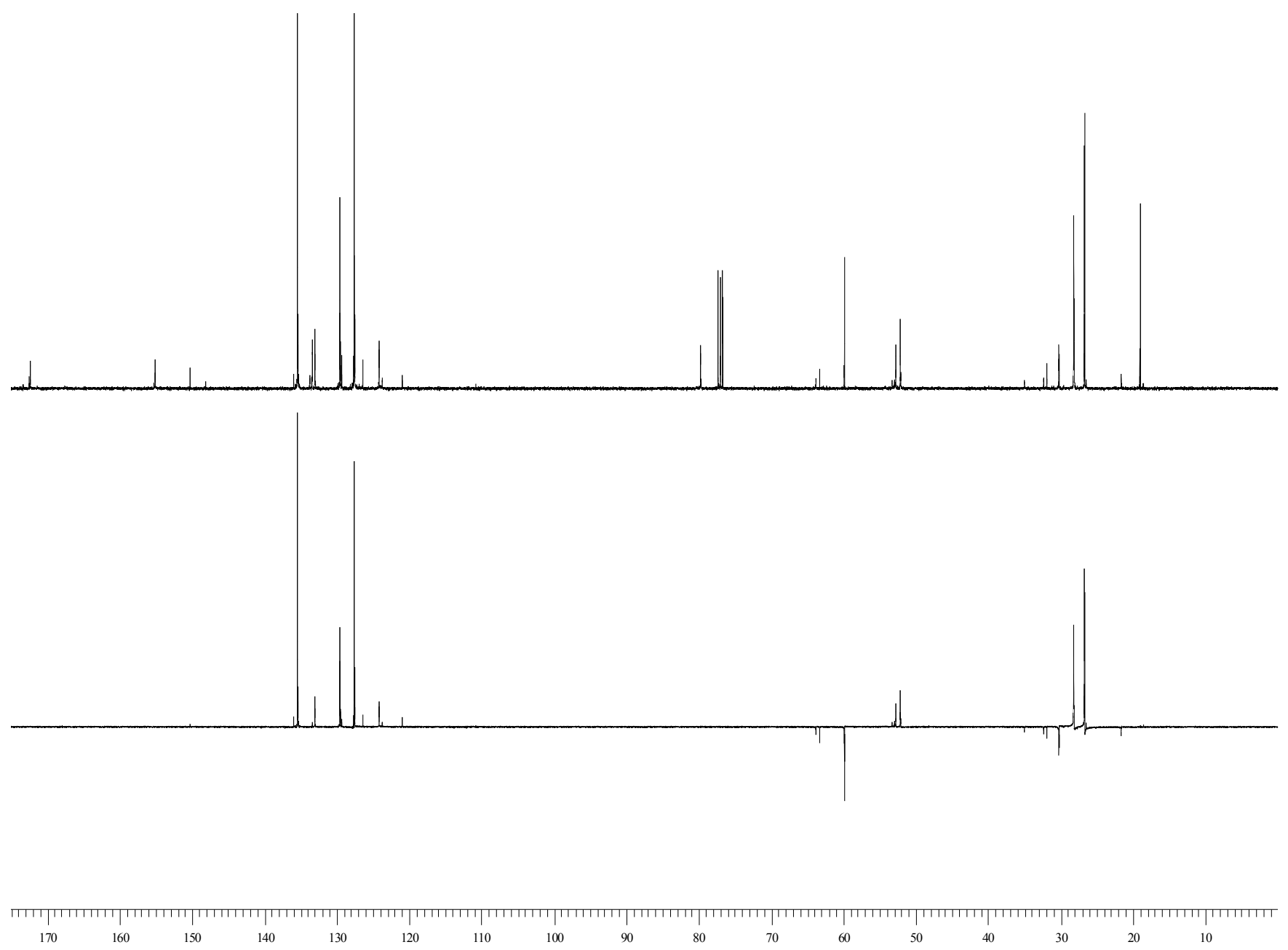

tert-Butyl

(Z)-5-\{[tert-butyl(diphenyl)silyl]oxy\}-1-(hydroxymethyl)-3,4-dimethyl-3-pentenylcarbamate 10

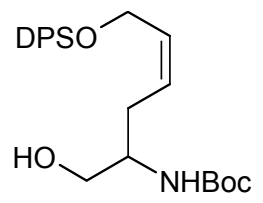

A solution of ester 9 (3.94 g, $7.93 \mathrm{mmol}, 70 \%$ pure containing 30\% of ester 11) was dissolved in anhydrous $\mathrm{MeOH}(100 \mathrm{~mL})$ and then cooled to $0{ }^{\circ} \mathrm{C} . \mathrm{NaBH}_{4}(2.90 \mathrm{~g}, 79.3 \mathrm{mmol})$ was added slowly in portions, the mixture was then allowed to stir at $\mathrm{rt}$ for $20 \mathrm{~h}$, TLC indicated that some starting material remained and a further portion of $\mathrm{NaBH}_{4}(1.00 \mathrm{~g}, 27.0 \mathrm{mmol})$ was added. The reaction was stirred for a further $2 \mathrm{~h}$ after which time TLC indicated the reaction to be complete. The reaction mixture was concentrated onto silica and purified by flash column chromatography (on silica) eluting with a solvent gradient of $\mathrm{Et}_{2} \mathrm{O}$ to $\mathrm{EtOAc}$ to $\mathrm{EtOAc} / \mathrm{MeOH}(9: 1)$ to afford alcohol 10 (3.41 g, 92\%) as colorless oil. 
From the ${ }^{1} \mathrm{H}$ NMR spectrum the purity of alcohol $\mathbf{1 0}$ was estimated to be approximately $70 \%$ containing $30 \%$ of alkane $\mathbf{1 2}$ (carried through from the over-reduction of alkyne $\mathbf{8}$ ) by comparison of the integrals values of the signals between 7.70-7.34 $(10 \mathrm{H})$ and $5.46(1 \mathrm{H}) \mathrm{ppm} . \mathrm{R}_{\mathrm{f}} 0.4$ (petroleum ether: $\mathrm{Et}_{2} \mathrm{O}$ 1:2); IR: (neat) $v_{\max } 3417,3361,2957,2930,2857,1688,1590,1503,1472,1427,1364,1169,1111 \mathrm{~cm}^{-1} ;{ }^{1} \mathrm{H}$ NMR (400 MHz, $\left.\mathrm{CDCl}_{3}\right) \delta_{\mathrm{H}}: 7.70-7.66(\mathrm{~m}, 4 \mathrm{H}), 7.43-7.34(\mathrm{~m}, 6 \mathrm{H}), 5.71$ (ddt, $J=11.0,6.5,1.0 \mathrm{~Hz}$, 1H), $5.46(\mathrm{ddt}, J=11.0,7.5,1.0 \mathrm{~Hz}, 1 \mathrm{H}), 4.85(\mathrm{~d}, J=8.0 \mathrm{~Hz}, 1 \mathrm{H}), 4.27(\mathrm{dd}, J=13.0,6.5 \mathrm{~Hz}, 1 \mathrm{H}), 4.19$ (dd, $J=13.0,6.5 \mathrm{~Hz}, 1 \mathrm{H}), 3.67-3.50(\mathrm{~m}, 3 \mathrm{H}), 3.09(\mathrm{br} \mathrm{s}, 1 \mathrm{H}), 2.30-2.17(\mathrm{~m}, 1 \mathrm{H}), 2.16-2.08(\mathrm{~m}, 1 \mathrm{H})$, $1.41(\mathrm{~s}, 9 \mathrm{H}), 1.05(\mathrm{~s}, 9 \mathrm{H}) ;{ }^{13} \mathrm{C}$ NMR $\left(100 \mathrm{MHz}, \mathrm{CDCl}_{3}\right) \delta_{\mathrm{C}}: 155.7(\mathrm{C}), 135.1(\mathrm{CH}), 133.1(\mathrm{C}), 131.0$ $(\mathrm{CH}), 129.3(\mathrm{CH}), 127.3(\mathrm{CH}), 127.2(\mathrm{CH}), 79.5(\mathrm{C}), 64.6\left(\mathrm{CH}_{2}\right), 59.8\left(\mathrm{CH}_{2}\right), 52.1(\mathrm{CH}), 29.3\left(\mathrm{CH}_{2}\right)$, $28.3\left(\mathrm{CH}_{3}\right), 26.7\left(\mathrm{CH}_{3}\right), 19.3(\mathrm{C})$; LRMS (CI, $\left.\mathrm{NH}_{3}\right) \mathrm{m} / z 470\left([\mathrm{M}+\mathrm{H}]^{+}, 5 \%\right),\left([\mathrm{M}-\mathrm{Boc}] \mathrm{H}^{+}, 100\right) ; \mathrm{HRMS}$ (CI, $\mathrm{NH}_{3}$ ) $m / z[\mathrm{M}+\mathrm{H}]^{+}$found 470.2720 ; calcd 470.2727 for $\mathrm{C}_{27} \mathrm{H}_{39} \mathrm{NO}_{4} \mathrm{Si}$. 


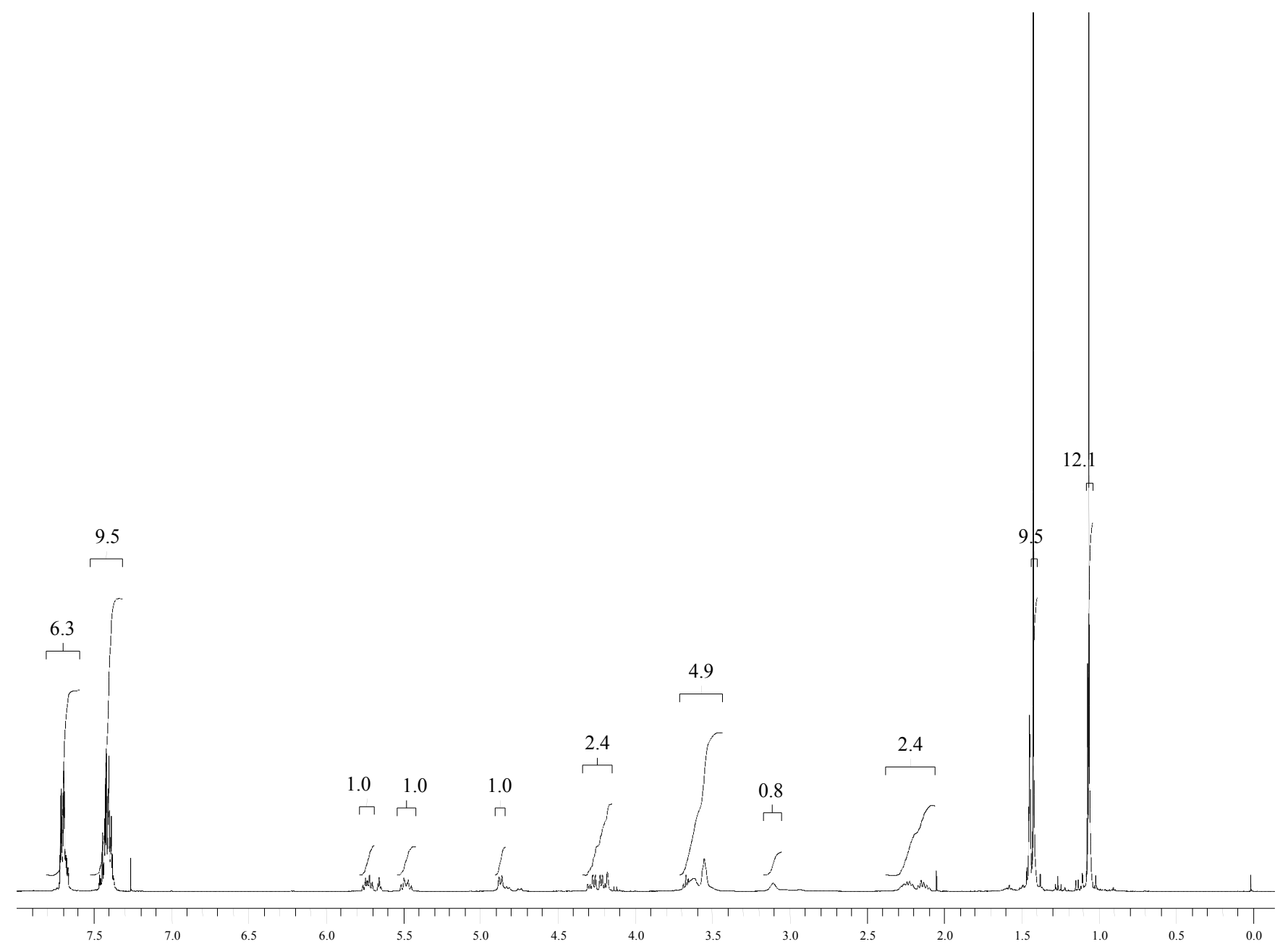




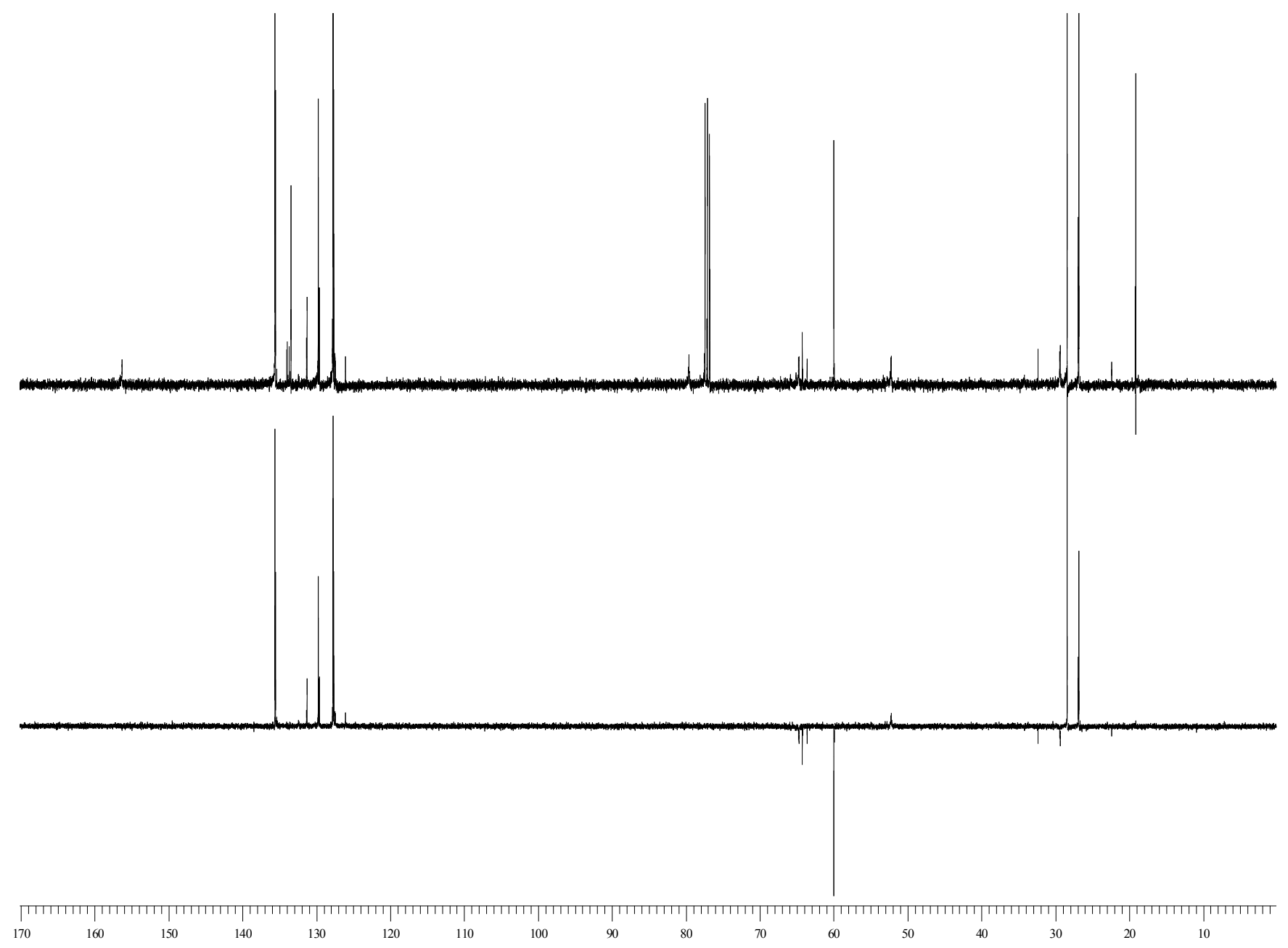

\section{3-Allyl-4-((Z)-4-\{[tert-butyl(diphenyl)silyl]oxy\}-2-butenyl)-1,3-oxazolidin-2-one 5}

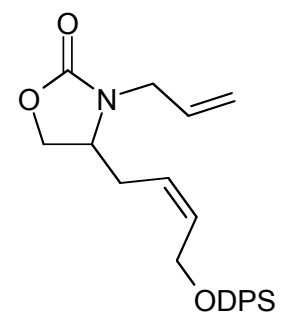

A solution of alcohol $10(10.0 \mathrm{~g}, 21.4 \mathrm{mmol}, 70 \%$ pure $)$ in anhydrous THF $(100 \mathrm{~mL})$ was cooled to $0{ }^{\circ} \mathrm{C}$ and $\mathrm{NaH}(3.08 \mathrm{~g}, 64.2 \mathrm{mmol})$ was added cautiously. The reaction mixture was stirred at $\mathrm{rt}$ for $24 \mathrm{~h}$ after which time allyl bromide $(5.56 \mathrm{~mL}, 64.2 \mathrm{mmol})$ was added and then stirring was continued for a further $16 \mathrm{~h}$. After addition of $\mathrm{NH}_{4} \mathrm{Cl}(700 \mathrm{~mL})$ the reaction mixture was extracted with $\mathrm{Et}_{2} \mathrm{O}(3 \times 300 \mathrm{~mL})$ and the combined organic phases were dried $\left(\mathrm{MgSO}_{4}\right)$, filtered and evaporated. Purification by flash column chromatography (on silica) using petroleum ether/ $\mathrm{Et}_{2} \mathrm{O}$ (3:1 to 2:1) as eluent afforded diene $\mathbf{5}$ (5.40 $\mathrm{g}$, 
$58 \%$ ) as a colorless oil. From the ${ }^{1} \mathrm{H}$ NMR spectrum the purity of diene $\mathbf{5}$ was estimated to be approximately $75 \%$ containing $25 \%$ of alkene $\mathbf{1 3}$ (carried through from the over-reduction of alkyne 8) by comparison of the integrals values of the signals between 7.70-7.57 (4H) and 5.85-5.69 (2H) ppm. $\mathrm{R}_{\mathrm{f}} 0.4$ (petroleum ether:Et $\mathrm{O}_{2} \mathrm{O}$ 1:1); IR: $\left(\mathrm{CH}_{2} \mathrm{Cl}_{2}\right) v_{\max } 3054,2960,2932,2859,1648,1585,1427,1263$ $\mathrm{cm}^{-1} ;{ }^{1} \mathrm{H}$ NMR $\left(400 \mathrm{MHz}, \mathrm{CDCl}_{3}\right) \delta_{\mathrm{H}}:$ 7.70-7.67 (m, 4H), 7.46-7.36 (m, 6H), 5.85-5.78 (m, 1H), 5.69 (dddd, $J=17.5,10.0,7.5,5.0 \mathrm{~Hz}, 1 \mathrm{H}), 5.13-5.11(\mathrm{~m}, 3 \mathrm{H}), 4.24-4.19(\mathrm{~m}, 3 \mathrm{H}), 4.09$ (ddt, $J=15.5,5.0$, $1.5 \mathrm{~Hz}, 1 \mathrm{H}), 3.84(\mathrm{dd}, J=8.5,6.5 \mathrm{~Hz}, 1 \mathrm{H}), 3.74-3.64(\mathrm{~m}, 1 \mathrm{H}), 3.49(\mathrm{ddt}, J=15.5,7.5,1.0 \mathrm{~Hz}, 1 \mathrm{H})$ 2.28-2.21 (m, 1H), 2.12-2.02 (m, 1H), $1.05(\mathrm{~s}, 9 \mathrm{H}) ;{ }^{13} \mathrm{C}$ NMR $\left(100 \mathrm{MHz}, \mathrm{CDCl}_{3}\right) \delta_{\mathrm{C}}: 157.8(\mathrm{C}), 135.4$ $(\mathrm{CH}), 133.6(\mathrm{CH}), 133.3(\mathrm{C}), 132.0(\mathrm{CH}), 129.7(\mathrm{CH}), 127.6(\mathrm{CH}), 122.9(\mathrm{CH}), 118.6\left(\mathrm{CH}_{2}\right), 66.5$ $\left(\mathrm{CH}_{2}\right), 60.0\left(\mathrm{CH}_{2}\right), 53.9(\mathrm{CH}), 44.6\left(\mathrm{CH}_{2}\right), 29.6\left(\mathrm{CH}_{2}\right), 26.7\left(\mathrm{CH}_{3}\right), 19.0(\mathrm{C}) ; \mathrm{LRMS}\left(\mathrm{CI}, \mathrm{NH}_{3}\right) \mathrm{m} / z 453$ $\left(\left[\mathrm{M}+\mathrm{NH}_{4}\right]^{+}, 20 \%\right), 436\left(\mathrm{M}+\mathrm{H}^{+}, 27\right), 358(100) ; \mathrm{HRMS}\left(\mathrm{CI}, \mathrm{NH}_{3}\right) \mathrm{m} / z\left[\mathrm{M}+\mathrm{H}^{+}\right]$found 436.2295; calcd 436.2308 for $\mathrm{C}_{26} \mathrm{H}_{33} \mathrm{NO}_{3} \mathrm{Si}$.

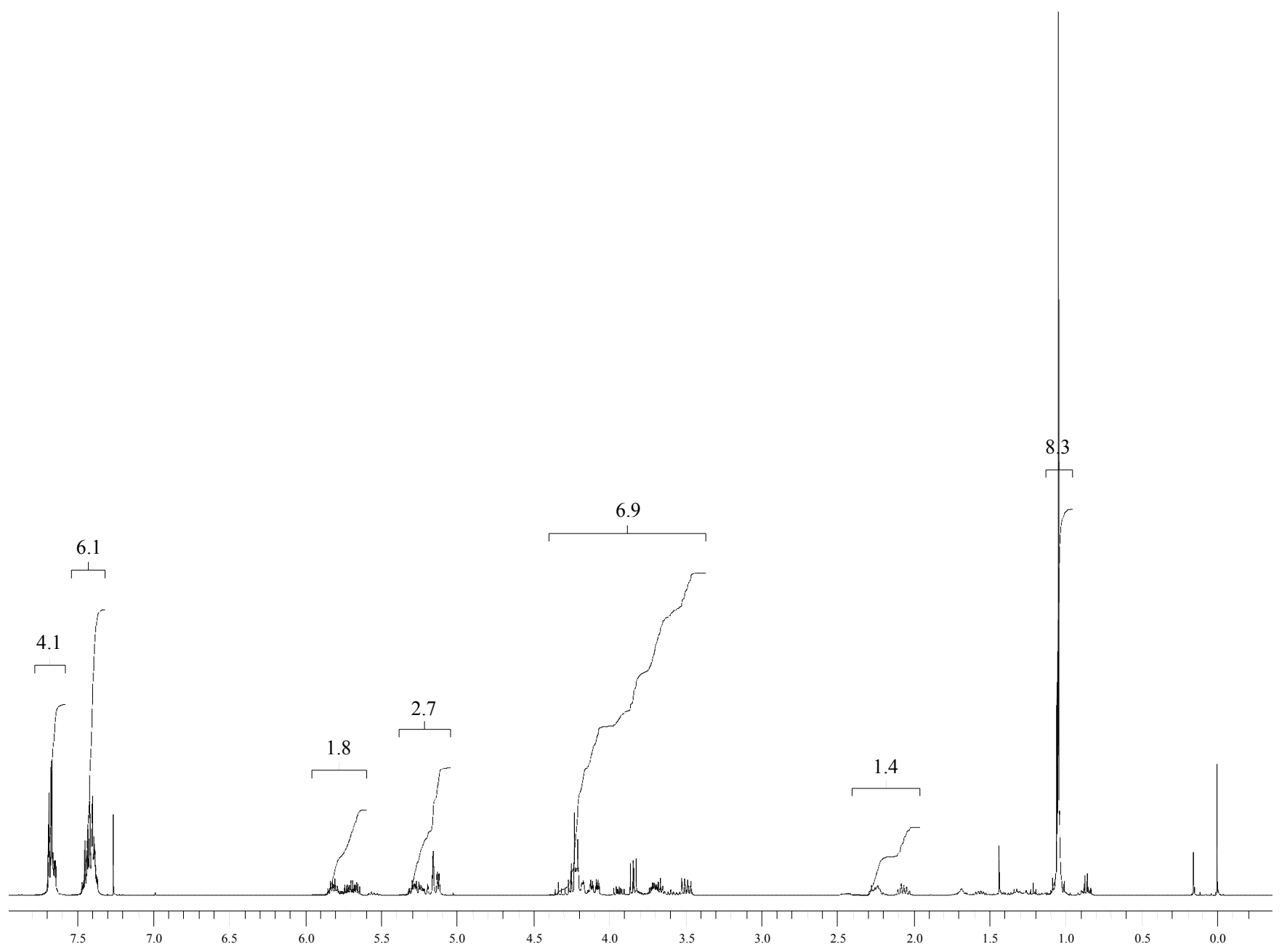



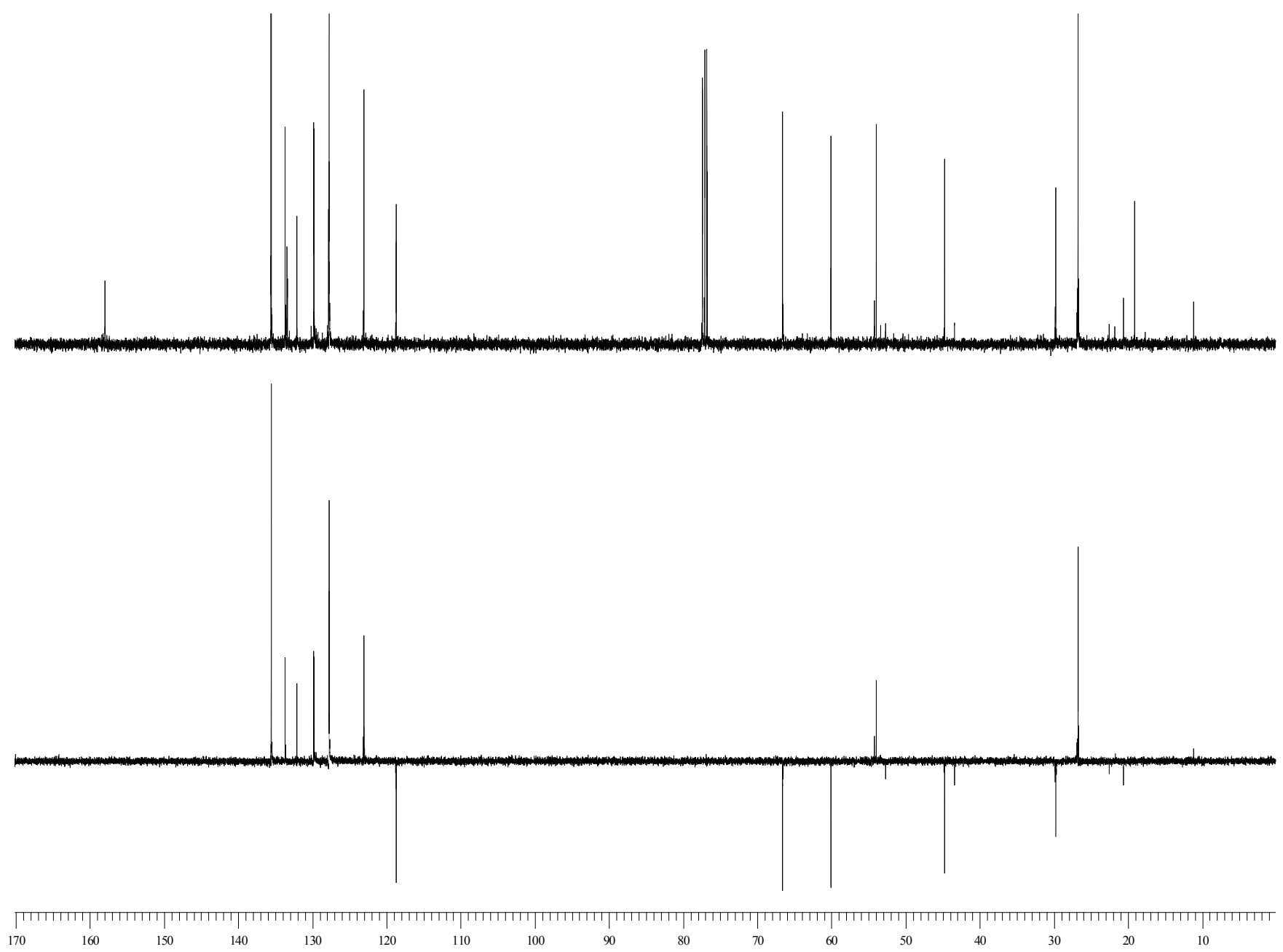

$O, O$-Diethyl [rel-(6S,7R,8aS)-7-(2-\{[tert-butyl(diphenyl)silyl] $]$ oxy\}ethyl)-3-oxohexahydro[1,3]oxazolo[3,4-a]pyridin-6-yl]methylphosphonothioate $4 a$ and $O, O$-diethyl $[r e l-(6 R, 7 R, 8 \mathrm{a} S)-7-(2-$ $\{[$ tert-butyl(diphenyl)silyl]oxy\}ethyl)-3-oxohexahydro[1,3]oxazolo[3,4-a]pyridin-6-yl]methylphosphonothioate 4b<smiles>CCOCCC1CC2COC(=O)N2CC1CP=S</smiles><smiles>CCOCCC1CC2COC(=O)N2C[C@H]1C[Pb](=S)OCC</smiles> 


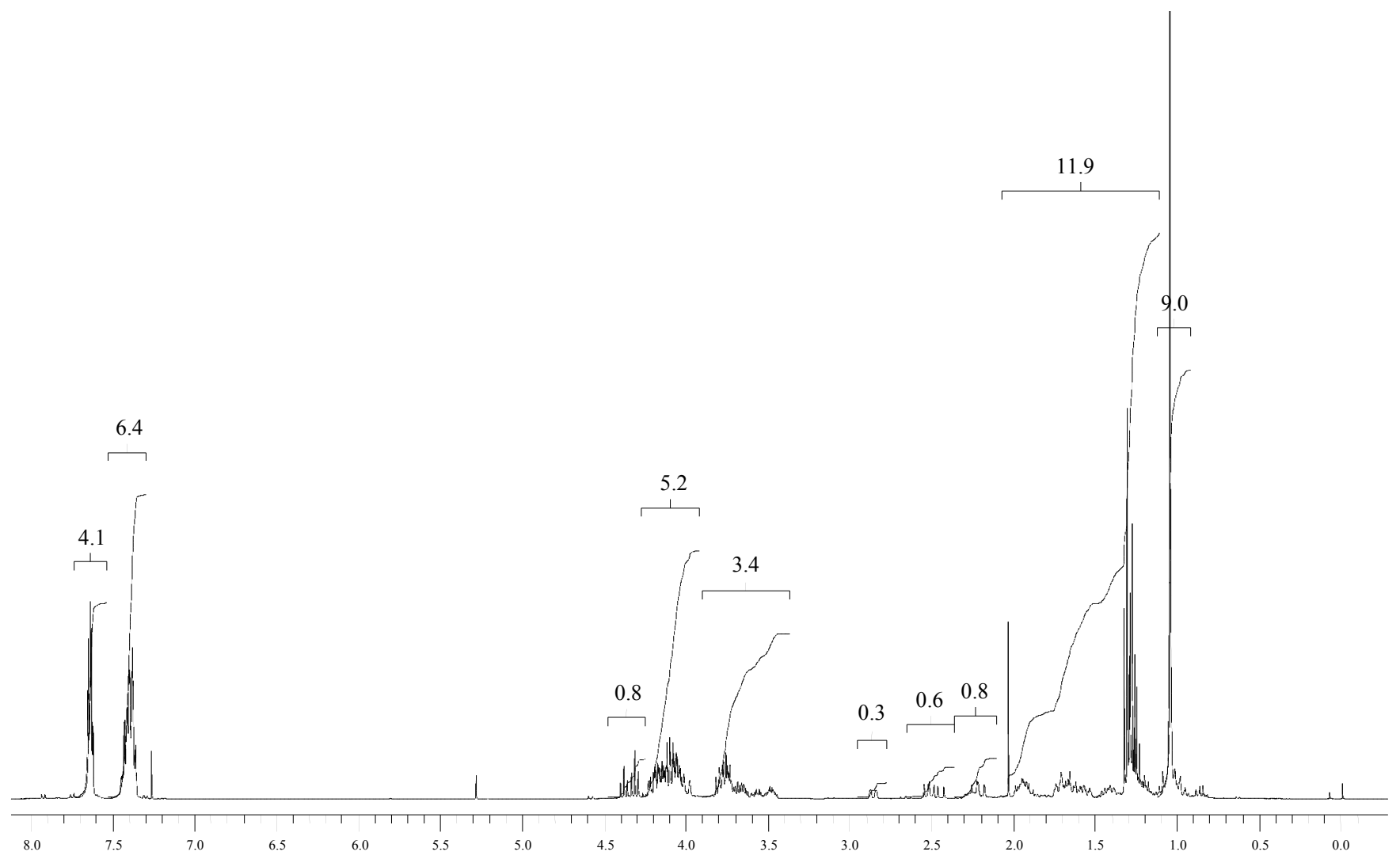




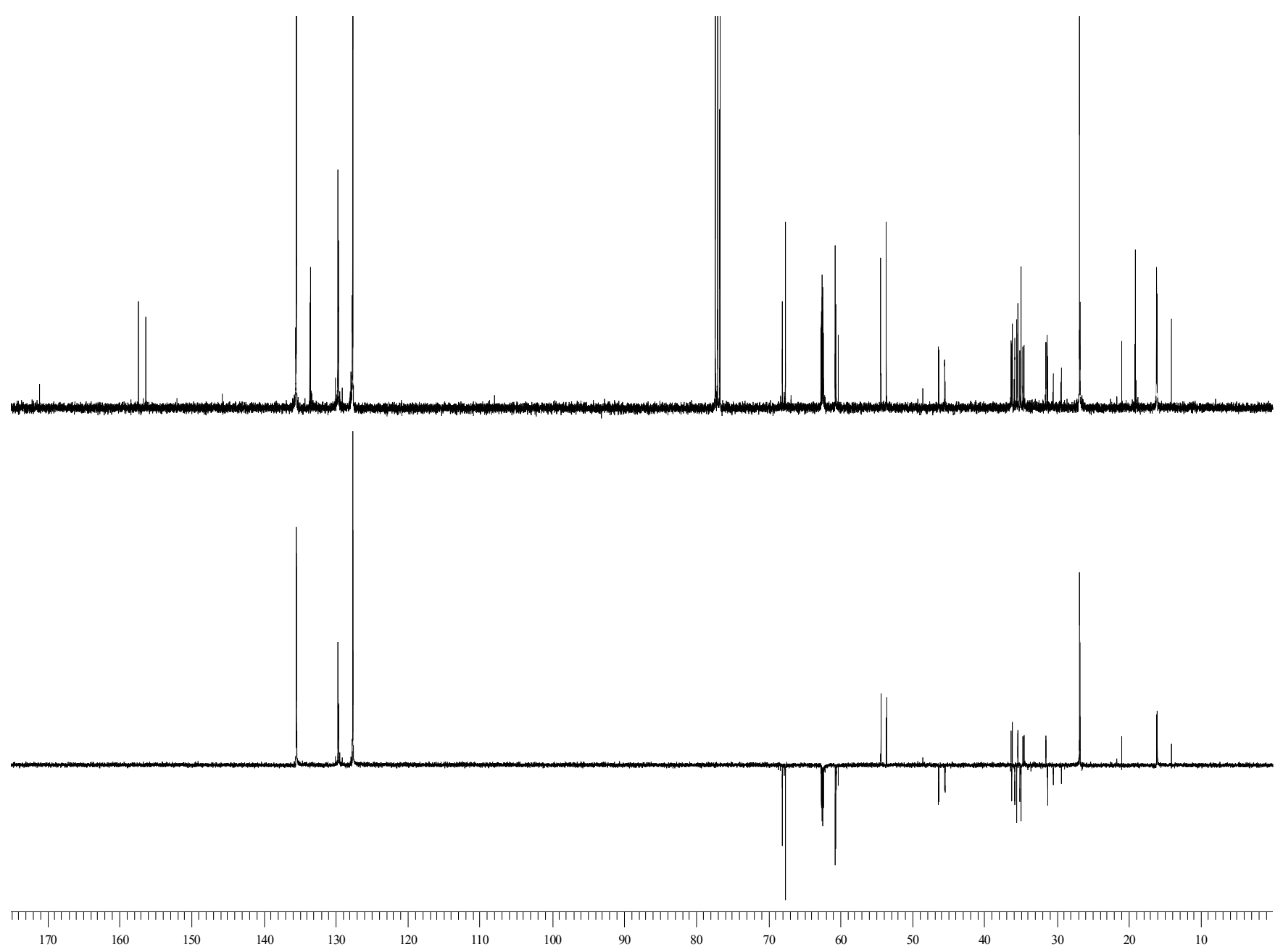

$O, O$-Diethyl $\quad[$ rel-(6S,7R,8aS)-7-(2-hydroxyethyl)-3-oxohexahydro[1,3]oxazolo[3,4-a]pyridin-6-yl]methylphosphonothioate $16 \mathrm{a}$ and $O, O$-diethyl [rel-(6R,7R,8aS)-7-(2-hydroxyethyl)-3-oxohexahydro[1,3]oxazolo[3,4-a]pyridin-6-yl]methylphosphonothioate 16b<smiles>CCOPSCC1CN2C(=O)OCC2CC1CCO</smiles><smiles>CCOP(C)SC[C@H]1CN2C(=O)OCC2CC1CCO</smiles>

To a solution of silyl ethers $\mathbf{4 a}$ and $\mathbf{4 b}(3.10 \mathrm{~g}, 5.26 \mathrm{mmol})$ in anhydrous THF (30 mL) was added a solution of TBAF (26.3 mL of a 1.0 M solution in THF, $26.3 \mathrm{mmol})$. The reaction mixture turned dark orange and was stirred at $\mathrm{rt}$ for $20 \mathrm{~h}$. After this time $\mathrm{NH}_{4} \mathrm{Cl}$ (saturated aqueous solution) was added, which was subsequently extracted with EtOAc. The combined organic phases were dried $\left(\mathrm{MgSO}_{4}\right)$, filtered and evaporated. The resulting orange residue was then purified by flash column chromatography (on silica) using a solvent gradient from $\mathrm{Et}_{2} \mathrm{O}$ /petroleum ether (3:1) to $\mathrm{Et}_{2} \mathrm{O}$ to $\mathrm{EtOAc}$ 
to afford the desired alcohol $16(1.42 \mathrm{~g}, 77 \%)$ as an inseparable mixture of two diastereomers as an orange oil. Both diastereomers: $\mathrm{R}_{\mathrm{f}} 0.4$ (EtOAc); IR: (neat) $v_{\max }$ 3446, 2981, 2930, 2873, 1741, 1478, 1431, 1389, 1272, 1160, 1098, 1049, 1023, $956 \mathrm{~cm}^{-1} ;{ }^{1} \mathrm{H}$ NMR (400 MHz, $\left.\mathrm{CDCl}_{3}\right) \delta_{\mathrm{H}}: 4.46$ and $4.42(2$ $\times \mathrm{t}, J=8.5 \mathrm{~Hz}, 1 \mathrm{H}), 4.23-3.99(\mathrm{~m}, 6 \mathrm{H}), 3.93-3.87(\mathrm{~m}, 1 \mathrm{H}), 3.77-3.60(\mathrm{~m}, 3 \mathrm{H}), 3.12($ br s, $1 \mathrm{H}), 2.96$ and $2.60(\mathrm{~d}, J=11.5 \mathrm{~Hz}$ and $\mathrm{dd}, J=13.5,10.5 \mathrm{~Hz}, 1 \mathrm{H}), 2.38-1.23(\mathrm{~m}, 1 \mathrm{H}), 2.09-1.43(\mathrm{~m}, 6 \mathrm{H}), 1.37-1.23(\mathrm{~m}$ $6 \mathrm{H}) ;{ }^{13} \mathrm{C} \mathrm{NMR}\left(100 \mathrm{MHz}, \mathrm{CDCl}_{3}\right) \delta_{\mathrm{C}}: 157.3(\mathrm{C}), 156.3(\mathrm{C}), 67.9\left(\mathrm{CH}_{2}\right), 67.6\left(\mathrm{CH}_{2}\right), 62.3\left(\mathrm{~d}, J_{\mathrm{CP}}=7.0\right.$ $\left.\mathrm{Hz}, \mathrm{CH}_{2}\right), 62.23\left(\mathrm{~d}, J_{\mathrm{CP}}=7.5 \mathrm{~Hz}, \mathrm{CH}_{2}\right), 62.15\left(\mathrm{~d}, J_{\mathrm{CP}}=7.0 \mathrm{~Hz}, \mathrm{CH}_{2}\right), 62.1\left(\mathrm{~d}, J_{\mathrm{CP}}=7.0 \mathrm{~Hz}, \mathrm{CH}_{2}\right), 58.9$ $\left(\mathrm{CH}_{2}\right), 58.8\left(\mathrm{CH}_{2}\right), 54.0(\mathrm{CH}), 53.4(\mathrm{CH}), 45.9\left(\mathrm{~d}, J_{\mathrm{CP}}=2.5 \mathrm{~Hz}, \mathrm{CH}_{2}\right), 45.1\left(\mathrm{CH}_{2}\right), 35.9\left(\mathrm{~d}, J_{\mathrm{CP}}=15.5\right.$ $\mathrm{Hz}, \mathrm{CH}), 35.6\left(\mathrm{~d}, J_{\mathrm{CP}}=1.5 \mathrm{~Hz}, \mathrm{CH}_{2}\right), 35.3\left(\mathrm{CH}_{2}\right), 35.2\left(\mathrm{~d}, J_{\mathrm{CP}}=4.0 \mathrm{~Hz}, \mathrm{CH}\right), 35.1\left(\mathrm{~d},{ }^{1} J_{\mathrm{CP}}=111.5 \mathrm{~Hz}\right.$ $\left.\mathrm{CH}_{2}\right), 34.6\left(\mathrm{CH}_{2}\right), 34.4\left(\mathrm{~d}, J_{\mathrm{CP}}=13.5 \mathrm{~Hz}, \mathrm{CH}\right), 31.2\left(\mathrm{CH}_{2}\right), 31.1\left(\mathrm{~d}, J_{\mathrm{CP}}=1.5 \mathrm{~Hz}, \mathrm{CH}\right), 29.5\left(\mathrm{~d}, J_{\mathrm{CP}}=\right.$ $\left.112.0 \mathrm{~Hz}, \mathrm{CH}_{2}\right), 15.81\left(\mathrm{~d}, J_{\mathrm{CP}}=7.0 \mathrm{~Hz}, \mathrm{CH}_{3}\right), 15.79\left(\mathrm{~d}, J_{\mathrm{CP}}=7.5 \mathrm{~Hz}, \mathrm{CH}_{3}\right) ; \mathrm{LRMS}\left(\mathrm{CI}, \mathrm{NH}_{3}\right) m / z 352$ $\left(\mathrm{M}+\mathrm{H}^{+}, 100 \%\right)$; HRMS $\left(\mathrm{CI}, \mathrm{NH}_{3}\right) \mathrm{m} / z\left[\mathrm{M}+\mathrm{H}^{+}\right.$found 352.1342 ; calcd 352.1348 for $\mathrm{C}_{14} \mathrm{H}_{26} \mathrm{NO}_{5} \mathrm{PSSi}$.

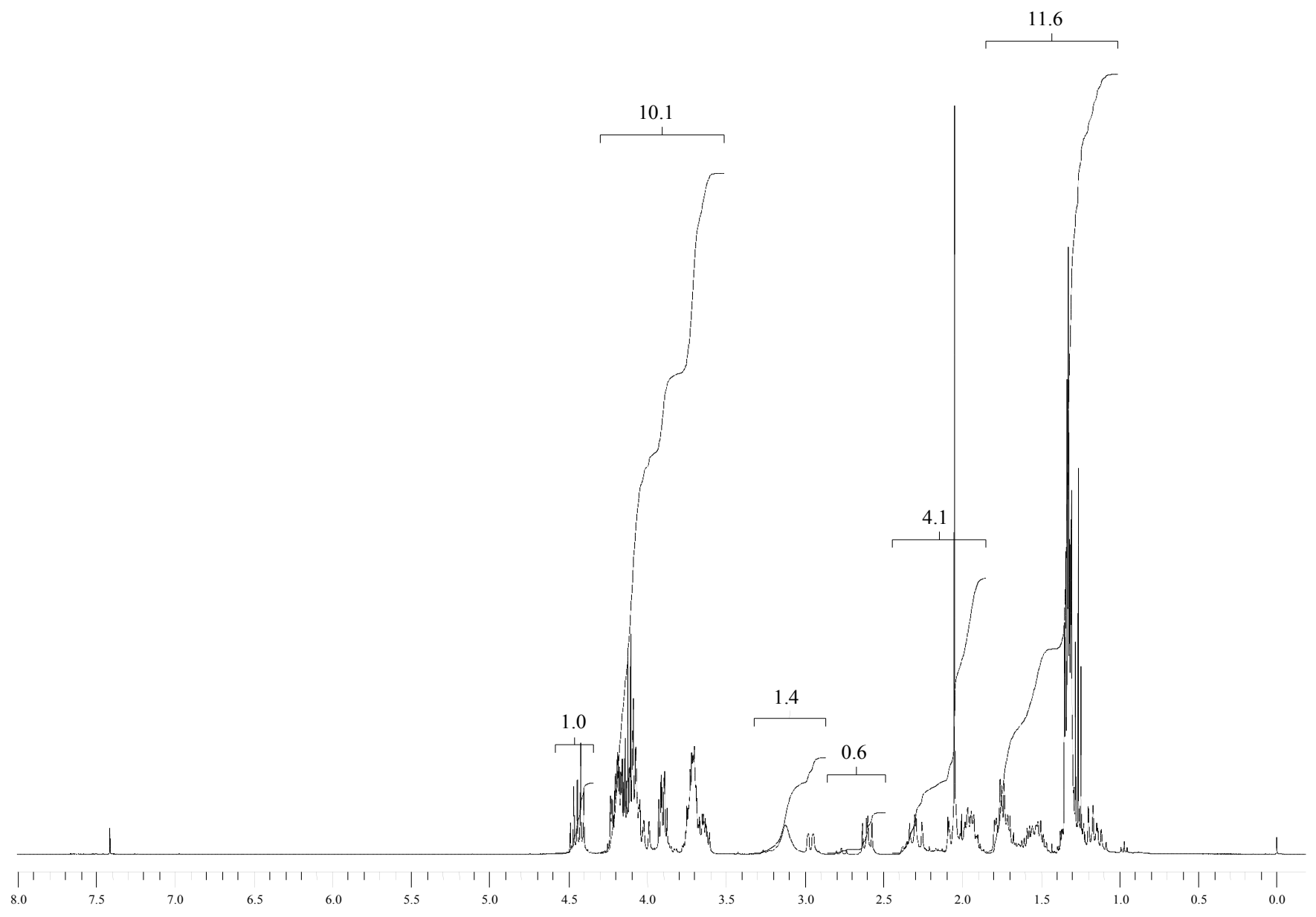



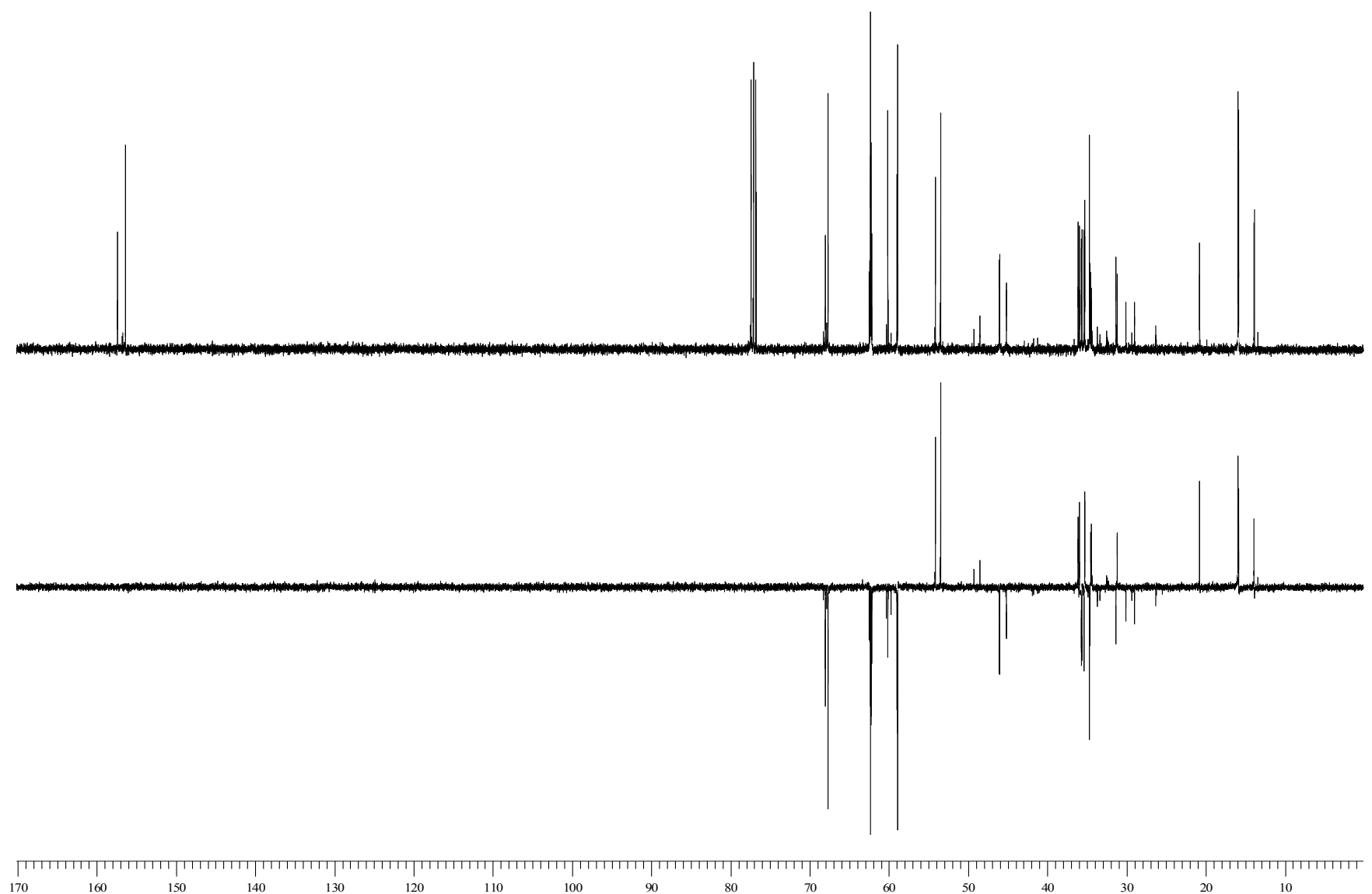

$O, O$-Diethyl $\quad\{r e l-(6 S, 7 R, 8 \mathrm{~S})$-3-oxo-7-[2-(trityloxy)ethyl]hexahydro[1,3]oxazolo[3,4-a]pyridin-6yl\}methylphosphonothioate $17 \mathrm{a}$ and $O, O$-diethyl $\{r e l-(6 R, 7 R, 8 \mathrm{a} S)-3-0 x 0-7-[2-(t r i t y l o x y)$ ethyl]hexahydro[1,3]oxazolo[3,4-a]pyridin-6-yl\}methylphosphonothioate 17b<smiles>CCOCCC1CC2COC(=O)N2CC1COC(OCC)c1ccccc1</smiles><smiles>CCOCCC1CC2COC(=O)N2CC1CCOC(c1ccccc1)(c1ccccc1)c1ccccc1</smiles>

A solution of alcohols $16 \mathbf{a}$ and $16 \mathbf{b}(0.50 \mathrm{~g}, 1.42 \mathrm{mmol})$ and $\operatorname{TrCl}(0.48 \mathrm{~g}, 1.71 \mathrm{mmol})$ in anhydrous $\mathrm{CH}_{2} \mathrm{Cl}_{2}(30 \mathrm{~mL})$ was cooled to $0{ }^{\circ} \mathrm{C}$. $\mathrm{Et}_{3} \mathrm{~N}(0.30 \mathrm{~mL}, 2.13 \mathrm{mmol})$ and DMAP $(0.01 \mathrm{~g}, 0.071 \mathrm{mmol})$ were added and the reaction was stirred at $\mathrm{rt}$ for 3 days. $\mathrm{NH}_{4} \mathrm{Cl}$ (saturated aqueous solution) was added and then extracted with $\mathrm{CH}_{2} \mathrm{Cl}_{2}$. The combined organic phases were dried $\left(\mathrm{MgSO}_{4}\right)$, filtered and evaporated. Purification by flash column chromatography (on silica) using petroleum ether/ $\mathrm{Et}_{2} \mathrm{O}$ (1:2) to $\mathrm{Et}_{2} \mathrm{O}$ as eluent afforded trityl ethers $\mathbf{1 7} \mathbf{a}$ and $\mathbf{1 7} \mathbf{b}(0.60 \mathrm{~g}, 71 \%)$ as an inseparable mixture as a white gum. Both 
diastereomers: $\mathrm{R}_{\mathrm{f}} 0.3$ (petroleum ether:Et $\mathrm{t}_{2} \mathrm{O} 1: 2$ ); IR: (neat) $v_{\max } 3060,2983,1753,1604,1491,1479$, 1448, 1427, 1236, 1049, 1027, $958 \mathrm{~cm}^{-1} ;{ }^{1} \mathrm{H}$ NMR (400 MHz, $\left.\mathrm{CDCl}_{3}\right) \delta_{\mathrm{H}}: 7.45-7.17(\mathrm{~m}, 15 \mathrm{H}), 4.32$ and $4.29(2 \times \mathrm{t}, J=8.5 \mathrm{~Hz}, 1 \mathrm{H}), 4.22-3.96(\mathrm{~m}, 5 \mathrm{H}), 3.75$ and $3.69(\mathrm{t}, J=8.5 \mathrm{~Hz}$ and dd, $J=8.5,6.0 \mathrm{~Hz}, 1 \mathrm{H})$, 3.55-3.42 (m, 1H), 3.29-3.13 and $3.05(\mathrm{~m}$, and td, $J=9.0,6.0 \mathrm{~Hz}, 2 \mathrm{H}), 2.85$ and $2.54(\mathrm{~d}, J=13.0 \mathrm{~Hz}$ and dd, $J=13.0,10.5 \mathrm{~Hz}, 1 \mathrm{H}), 2.32-1.35(\mathrm{~m}, 7 \mathrm{H}), 1.31,1.27$ and $1.26(\mathrm{t}, J=7.0 \mathrm{~Hz}, 6 \mathrm{H}), 1.00-0.86(\mathrm{~m}$, 1H); ${ }^{13} \mathrm{C}$ NMR (100 MHz, $\left.\mathrm{CDCl}_{3}\right) \delta_{\mathrm{C}}: 157.2(\mathrm{C}), 156.2(\mathrm{C}), 143.8(\mathrm{C}), 128.3(\mathrm{CH}), 127.6(\mathrm{CH}), 126.80$ $\mathrm{CH}), 126.76(\mathrm{CH}), 86.4(\mathrm{C}), 86.3(\mathrm{C}), 67.9\left(\mathrm{CH}_{2}\right), 67.4\left(\mathrm{CH}_{2}\right), 62.5\left(\mathrm{~d}, J_{\mathrm{CP}}=7.0 \mathrm{~Hz}, \mathrm{CH}_{2}\right), 62.41\left(\mathrm{~d}, J_{\mathrm{CP}}\right.$ $\left.=7.5 \mathrm{~Hz}, \mathrm{CH}_{2}\right), 62.35\left(\mathrm{~d}, J_{\mathrm{CP}}=7.0 \mathrm{~Hz}, \mathrm{CH}_{2}\right), 62.2\left(\mathrm{~d}, J_{\mathrm{CP}}=7.5 \mathrm{~Hz}, \mathrm{CH}_{2}\right), 60.4\left(\mathrm{CH}_{2}\right), 60.2\left(\mathrm{CH}_{2}\right), 54.2$ $(\mathrm{CH}), 53.4(\mathrm{CH}), 46.1\left(\mathrm{~d}, J_{\mathrm{CP}}=2.5 \mathrm{~Hz}, \mathrm{CH}_{2}\right), 45.3\left(\mathrm{CH}_{2}\right), 36.5\left(\mathrm{~d}, J_{\mathrm{CP}}=14.5 \mathrm{~Hz}, \mathrm{CH}\right), 35.6\left(\mathrm{~d}, J_{\mathrm{CP}}=\right.$ 113.0 Hz, $\left.\mathrm{CH}_{2}\right), 35.53\left(\mathrm{~d}, J_{\mathrm{CP}}=4.0 \mathrm{~Hz}, \mathrm{CH}\right), 35.50\left(\mathrm{CH}_{2}\right), 35.1\left(\mathrm{~d}, J_{\mathrm{CP}}=13.5 \mathrm{~Hz}, \mathrm{CH}\right), 33.1\left(\mathrm{CH}_{2}\right), 32.4$ $\left(\mathrm{CH}_{2}\right), 31.6\left(\mathrm{~d}, J_{\mathrm{CP}}=2.5 \mathrm{~Hz}, \mathrm{CH}\right), 31.1\left(\mathrm{CH}_{2}\right), 29.8\left(\mathrm{~d}, J_{\mathrm{CP}}=112.0 \mathrm{~Hz}, \mathrm{CH}_{2}\right), 16.04\left(\mathrm{~d}, J_{\mathrm{CP}}=7.0 \mathrm{~Hz}\right.$, $\left.\mathrm{CH}_{3}\right), 16.00\left(\mathrm{~d}, J_{\mathrm{CP}}=7.0 \mathrm{~Hz}, \mathrm{CH}_{3}\right), 15.98\left(\mathrm{~d}, J_{\mathrm{CP}}=7.0 \mathrm{~Hz}, \mathrm{CH}_{3}\right), 15.95\left(\mathrm{~d}, J_{\mathrm{CP}}=7.0 \mathrm{~Hz}, \mathrm{CH}_{3}\right) ; \mathrm{LRMS}$ (FAB) $m / z\left(616\left[\mathrm{M}+\mathrm{Na}^{+}\right], 100 \%\right), 243$ (77); HRMS (FAB) $\mathrm{m} / z\left[\mathrm{M}+\mathrm{Na}^{+}\right]$found 616.2262; calcd 616.2263 for $\mathrm{C}_{33} \mathrm{H}_{41} \mathrm{NO}_{5} \mathrm{PS}$.

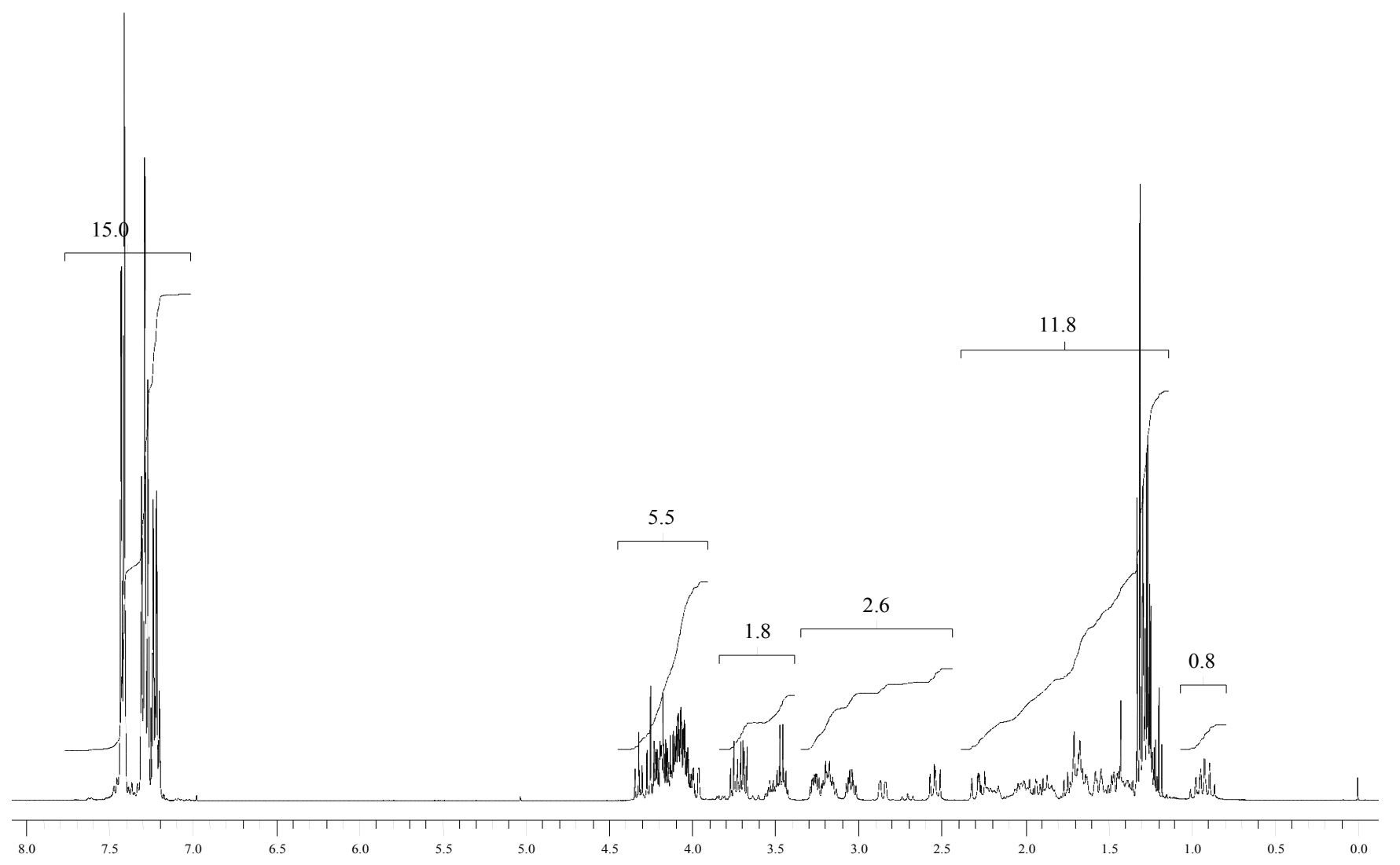




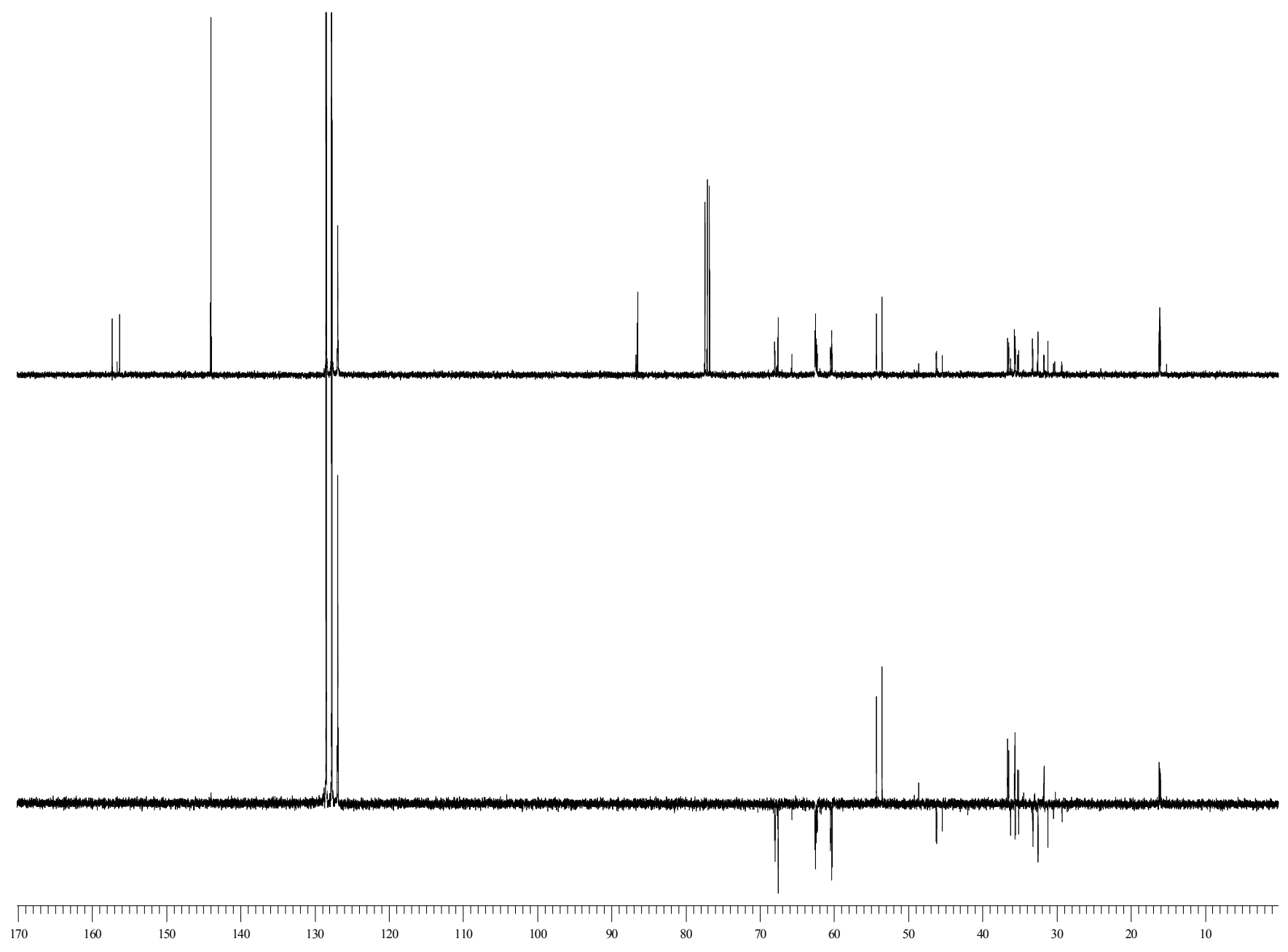

$O, O$-Diethyl $\quad\{$ rel-(3S,4R,6S)-6-(\{[tert-butyl(diphenyl)silyl $]$ oxy $\}$ methyl)-4-[2-(trityloxy)ethyl]piperidinyl\}methylphosphonothioate $\quad 18 \mathrm{a}$ and $O, O$-diethyl $\quad\{$ rel-(3R,4R,6S)-6-(\{[tert-butyl(diphenyl)silyl]oxy\}methyl)-4-[2-(trityloxy)ethyl]piperidinyl\}methylphosphonothioate 18b
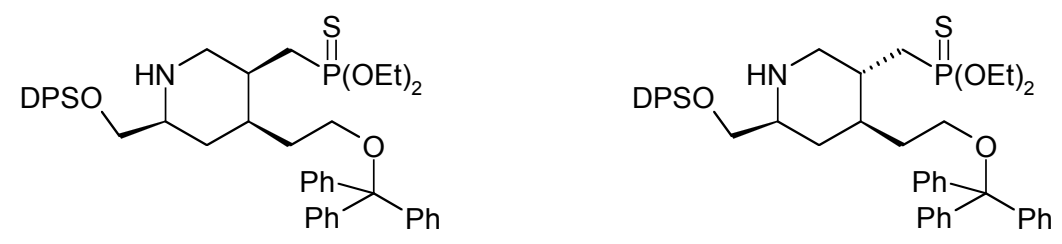

A mixture of $\mathbf{1 7} \mathbf{a}$ and $\mathbf{1 7 b}(1.10 \mathrm{~g}, 1.85 \mathrm{mmol})$ were dissolved in $\mathrm{EtOH} / \mathrm{H}_{2} \mathrm{O}(5: 1.120 \mathrm{~mL})$ and sodium hydroxide $(0.76 \mathrm{~g}, 18.5 \mathrm{mmol})$ was added and the reaction mixture was heated to reflux for $24 \mathrm{~h}$. After cooling to rt, the solvent was removed by evaporation to leave a white solid, which was washed with $\mathrm{CHCl}_{3}$. The combined organic phases were dried $\left(\mathrm{MgSO}_{4}\right)$, filtered and evaporated to give the crude amino alcohols as a white gum, which were dissolved in anhydrous $\mathrm{CH}_{2} \mathrm{Cl}_{2}(50 \mathrm{~mL})$. At $0{ }^{\circ} \mathrm{C}$, was 
added DPSCl $(0.58 \mathrm{~mL}, 2.2 \mathrm{mmol})$ and imidazole $(0.25 \mathrm{~g}, 3.70 \mathrm{mmol})$. The reaction was stirred at $\mathrm{rt}$ for $24 \mathrm{~h}$ and $\mathrm{NH}_{4} \mathrm{Cl}$ (saturated aqueous solution) was added followed by extraction using $\mathrm{CH}_{2} \mathrm{Cl}_{2}$. The combined organic phases were dried $\left(\mathrm{MgSO}_{4}\right)$, filtered and evaporated. Purification by flash column chromatography (on silica) using petroleum ether/Et ${ }_{2} \mathrm{O}(3: 1$ to $1: 1)$ as eluent afforded diastereomer 18a $(0.47 \mathrm{~g}, 31 \%)$ and diastereomer $\mathbf{1 8 b}(0.59 \mathrm{~g}, 40 \%)$ as colorless oils. 18a: $\mathrm{R}_{\mathrm{f}} 0.4$ (petroleum ether:Et ${ }_{2} \mathrm{O}$ 1:1); IR: (neat) $v_{\max } 3070,2929,2858,1590,1489,1471,1448,1427,1388,1256,1218,1157,1111$, 1066, 1051, 1029, $953 \mathrm{~cm}^{-1} ;{ }^{1} \mathrm{H}$ NMR (400 MHz, $\left.\mathrm{CDCl}_{3}\right) \delta_{\mathrm{H}}: 7.67-7.64(\mathrm{~m}, 4 \mathrm{H}), 7.43-7.35$ (m, 12H), 7.28-7.17 (m, 9H), 4.16-3.97 (m, 4H), $3.54(\mathrm{dd}, J=10.0,3.5 \mathrm{~Hz}, 1 \mathrm{H}), 3.43(\mathrm{dd}, J=10.0,7.5 \mathrm{~Hz}, 1 \mathrm{H})$, $3.31(\mathrm{dd}, J=12.0,1.5 \mathrm{~Hz}, 1 \mathrm{H}), 3.15-3.04(\mathrm{~m}, 2 \mathrm{H}), 2.71(\mathrm{~d}, J=12.0 \mathrm{~Hz}, 1 \mathrm{H}), 2.68-2.61(\mathrm{~m}, 1 \mathrm{H}), 2.44$ $(\mathrm{td}, J=15.5,10.5 \mathrm{~Hz}, 1 \mathrm{H}), 2.08-1.30(\mathrm{~m}, 7 \mathrm{H}), 1.27(\mathrm{t}, J=7.0 \mathrm{~Hz}, 3 \mathrm{H}), 1.26(\mathrm{t}, J=7.0 \mathrm{~Hz}, 3 \mathrm{H}), 1.04(\mathrm{~s}$, 9H), $0.74(\mathrm{q}, J=12.0 \mathrm{~Hz}, 1 \mathrm{H}) ;{ }^{13} \mathrm{C} \mathrm{NMR}\left(100 \mathrm{MHz}, \mathrm{CDCl}_{3}\right) \delta_{\mathrm{C}}: 144.3(\mathrm{C}), 135.52(\mathrm{CH}), 135.48(\mathrm{CH})$, $133.49(\mathrm{C}), 133.43(\mathrm{C}), 129.61(\mathrm{CH}), 129.58(\mathrm{CH}), 128.6(\mathrm{CH}), 127.7(\mathrm{CH}), 127.63(\mathrm{CH}), 127.61(\mathrm{CH})$, $126.8(\mathrm{CH}), 86.3(\mathrm{C}), 68.1\left(\mathrm{CH}_{2}\right), 62.1$ (app. t, $\left.J_{\mathrm{CP}}=7.0 \mathrm{~Hz}, \mathrm{CH}_{2}\right), 60.9\left(\mathrm{CH}_{2}\right), 58.0(\mathrm{CH}), 49.9\left(\mathrm{CH}_{2}\right)$, $36.5\left(\mathrm{~d}, J_{\mathrm{CP}}=15.5 \mathrm{~Hz}, \mathrm{CH}\right), 34.0\left(\mathrm{CH}_{2}\right), 33.0\left(\mathrm{~d}, J_{\mathrm{CP}}=1.5 \mathrm{~Hz}, \mathrm{CH}\right), 29.6\left(\mathrm{CH}_{2}\right), 29.5\left(\mathrm{~d}, J_{\mathrm{CP}}=109.5 \mathrm{~Hz}\right.$, $\left.\mathrm{CH}_{2}\right), 26.8\left(\mathrm{CH}_{3}\right), 19.2(\mathrm{C}), 16.17\left(\mathrm{~d}, J_{\mathrm{CP}}=8.5 \mathrm{~Hz}, \mathrm{CH}_{3}\right), 16.16\left(\mathrm{~d}, J_{\mathrm{CP}}=7.0 \mathrm{~Hz}, \mathrm{CH}_{3}\right)$; LRMS (FAB) m/z $828\left(\left[\mathrm{M}+\mathrm{Na}^{+}\right], 3 \%\right), 806(3), 243$ (100) 18b: $\mathrm{R}_{\mathrm{f}} 0.2$ (petroleum ether: $\mathrm{Et}_{2} \mathrm{O}$ 1:1); IR: (neat) $v_{\max } 3068$, 2931, 2858, 1591, 1489, 1448, 1429, 1388, 1265, 1159, 1111, 1052, $1030 \mathrm{~cm}^{-1} ;{ }^{1} \mathrm{H}$ NMR $(400 \mathrm{MHz}$, $\left.\mathrm{CDCl}_{3}\right) \delta_{\mathrm{H}}: 7.64-7.62(\mathrm{~m}, 4 \mathrm{H}), 7.42-7.33(\mathrm{~m}, 12 \mathrm{H}), 7.26-7.15(\mathrm{~m}, 9 \mathrm{H}), 4.20-4.01(\mathrm{~m}, 4 \mathrm{H}), 3.56(\mathrm{dd}, J=$ 11.5, $3.0 \mathrm{~Hz}, 1 \mathrm{H}), 3.46(\mathrm{dd}, J=10.0,3.5 \mathrm{~Hz}, 1 \mathrm{H}), 3.41(\mathrm{dd}, J=10.0,3.5 \mathrm{~Hz}, 1 \mathrm{H}), 3.22-3.17(\mathrm{~m}, 1 \mathrm{H})$, $2.96(\mathrm{td}, J=8.5,6.0 \mathrm{~Hz}, 1 \mathrm{H}), 2.67-2.54(\mathrm{~m}, 1 \mathrm{H}), 2.36(\mathrm{t}, J=11.5,1 \mathrm{H}), 2.25(\mathrm{dd}, J=18.0,12.0 \mathrm{~Hz}, 1 \mathrm{H})$, $2.14($ br s, $1 \mathrm{H}), 1.99(\mathrm{dt}, J=12.0,7.5 \mathrm{~Hz}, 1 \mathrm{H}), 1.72-1.62(\mathrm{~m}, 2 \mathrm{H}), 1.31(\mathrm{t}, J=7.5 \mathrm{~Hz}, 3 \mathrm{H}), 1.30(\mathrm{t}, J=$ $7.5 \mathrm{~Hz}, 3 \mathrm{H}), 1.27-1.19(\mathrm{~m}, 3 \mathrm{H}), 1.03(\mathrm{~s}, 9 \mathrm{H}), 0.64(\mathrm{q}, J=12.5 \mathrm{~Hz}, 1 \mathrm{H}) ;{ }^{13} \mathrm{C} \mathrm{NMR}\left(100 \mathrm{MHz}, \mathrm{CDCl}_{3}\right)$ $\delta_{\mathrm{C}}: 144.1(\mathrm{C}), 135.5(\mathrm{CH}), 135.4(\mathrm{CH}), 133.4(\mathrm{C}), 133.2(\mathrm{C}), 129.6(\mathrm{CH}), 129.5(\mathrm{CH}), 128.5(\mathrm{CH})$, $127.60(\mathrm{CH}), 127.57(\mathrm{CH}), 126.8(\mathrm{CH}), 86.3(\mathrm{C}), 68.1\left(\mathrm{CH}_{2}\right), 62.3\left(\mathrm{~d}, J_{\mathrm{CP}}=7.0 \mathrm{~Hz}, \mathrm{CH}_{2}\right), 62.0\left(\mathrm{~d}, J_{\mathrm{CP}}=\right.$ $\left.7.0 \mathrm{~Hz}, \mathrm{CH}_{2}\right), 60.5\left(\mathrm{CH}_{2}\right), 57.7(\mathrm{CH}), 38.1\left(\mathrm{~d}, J_{\mathrm{CP}}=15.5 \mathrm{~Hz}, \mathrm{CH}\right), 37.3\left(\mathrm{~d}, J_{\mathrm{CP}}=1.5 \mathrm{~Hz}, \mathrm{CH}\right), 36.2(\mathrm{~d}$, 
$\left.J_{\mathrm{CP}}=109.5 \mathrm{~Hz}, \mathrm{CH}_{2}\right), 33.3\left(\mathrm{CH}_{2}\right), 29.0\left(\mathrm{CH}_{2}\right), 26.8\left(\mathrm{CH}_{3}\right), 19.1(\mathrm{C}), 16.17\left(\mathrm{~d}, J_{\mathrm{CP}}=7.5 \mathrm{~Hz}, \mathrm{CH}_{3}\right), 16.14$ $\left(\mathrm{d}, J_{\mathrm{CP}}=7.0 \mathrm{~Hz}, \mathrm{CH}_{3}\right)$; LRMS (FAB) $m / z 828\left(\left[\mathrm{M}+\mathrm{Na}^{+}\right], 9 \%\right), 806$ (7), $243(100)$; HRMS (FAB) $m / z$ $\left[\mathrm{M}+\mathrm{H}^{+}\right]$found 806.3830; calcd 806.3828 for $\mathrm{C}_{40} \mathrm{H}_{60} \mathrm{NO}_{4} \mathrm{PSSi}$.

$18 a$

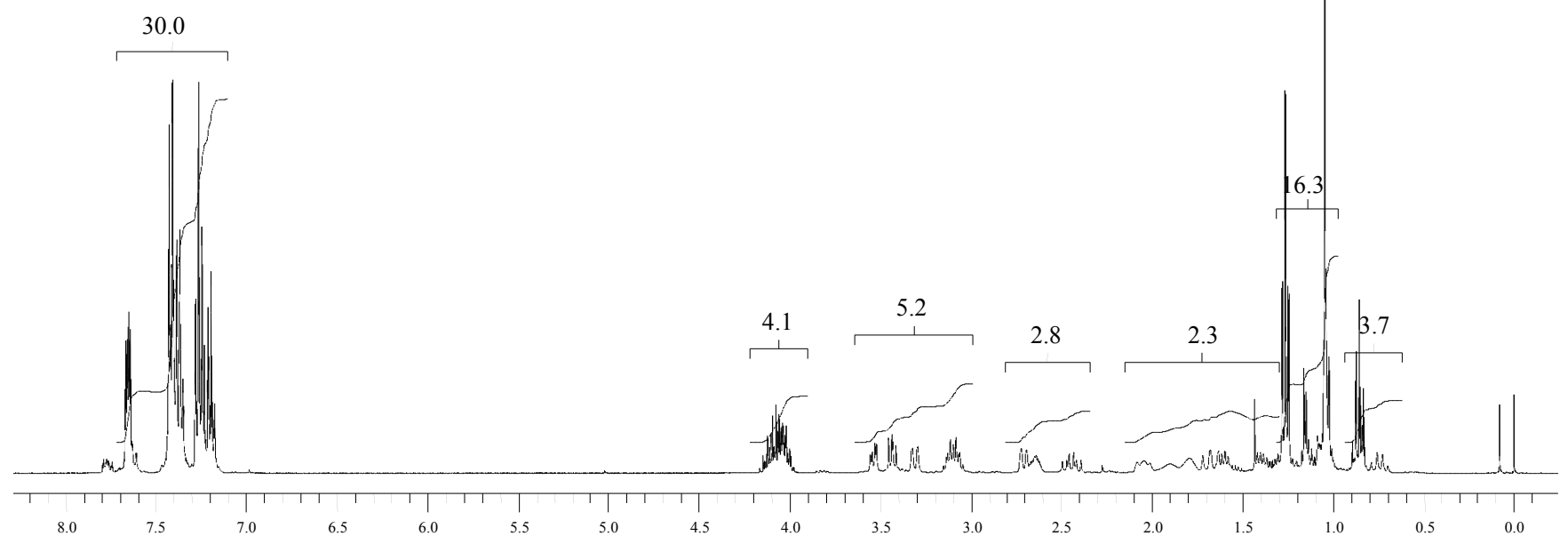



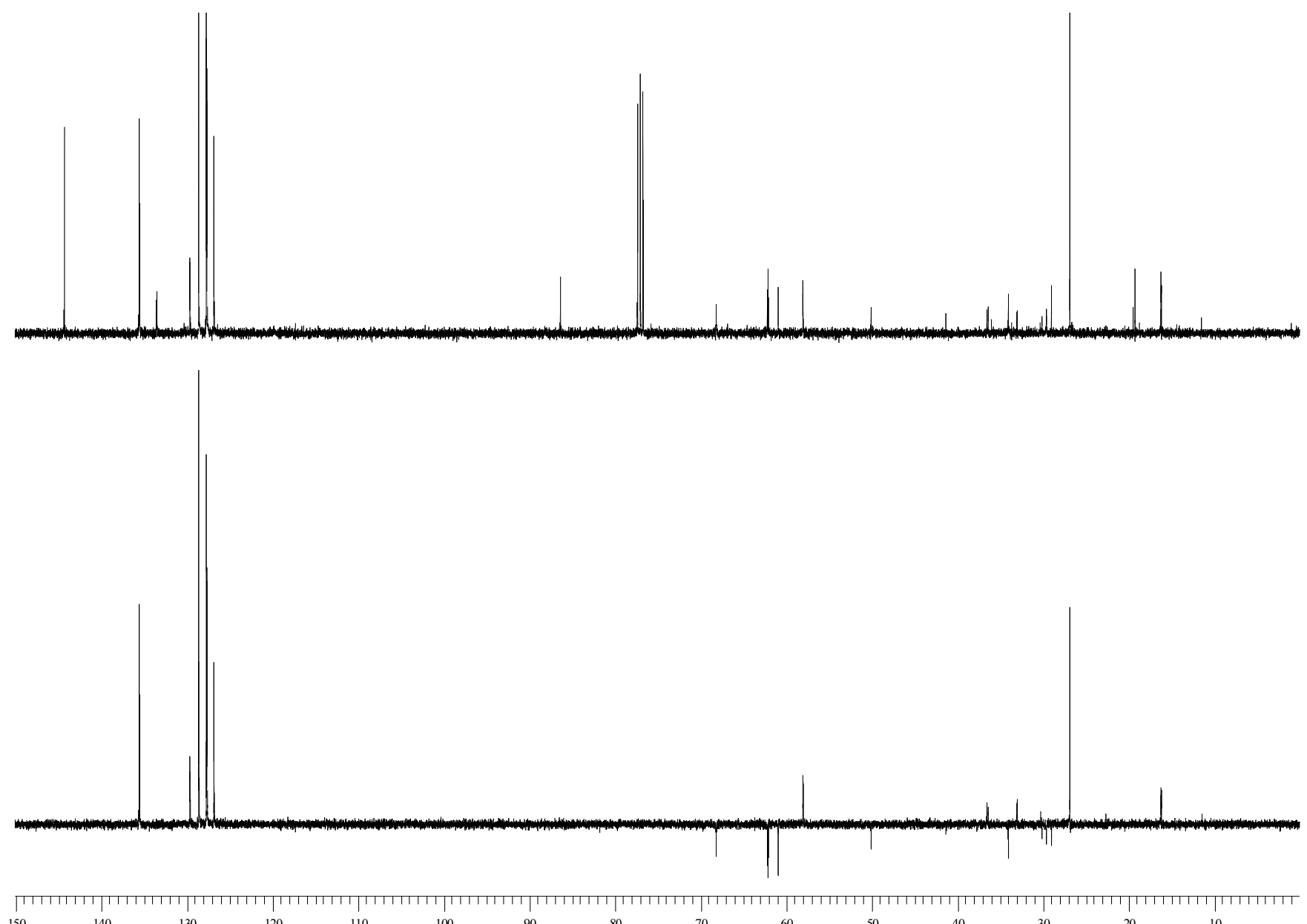

$18 b$ 

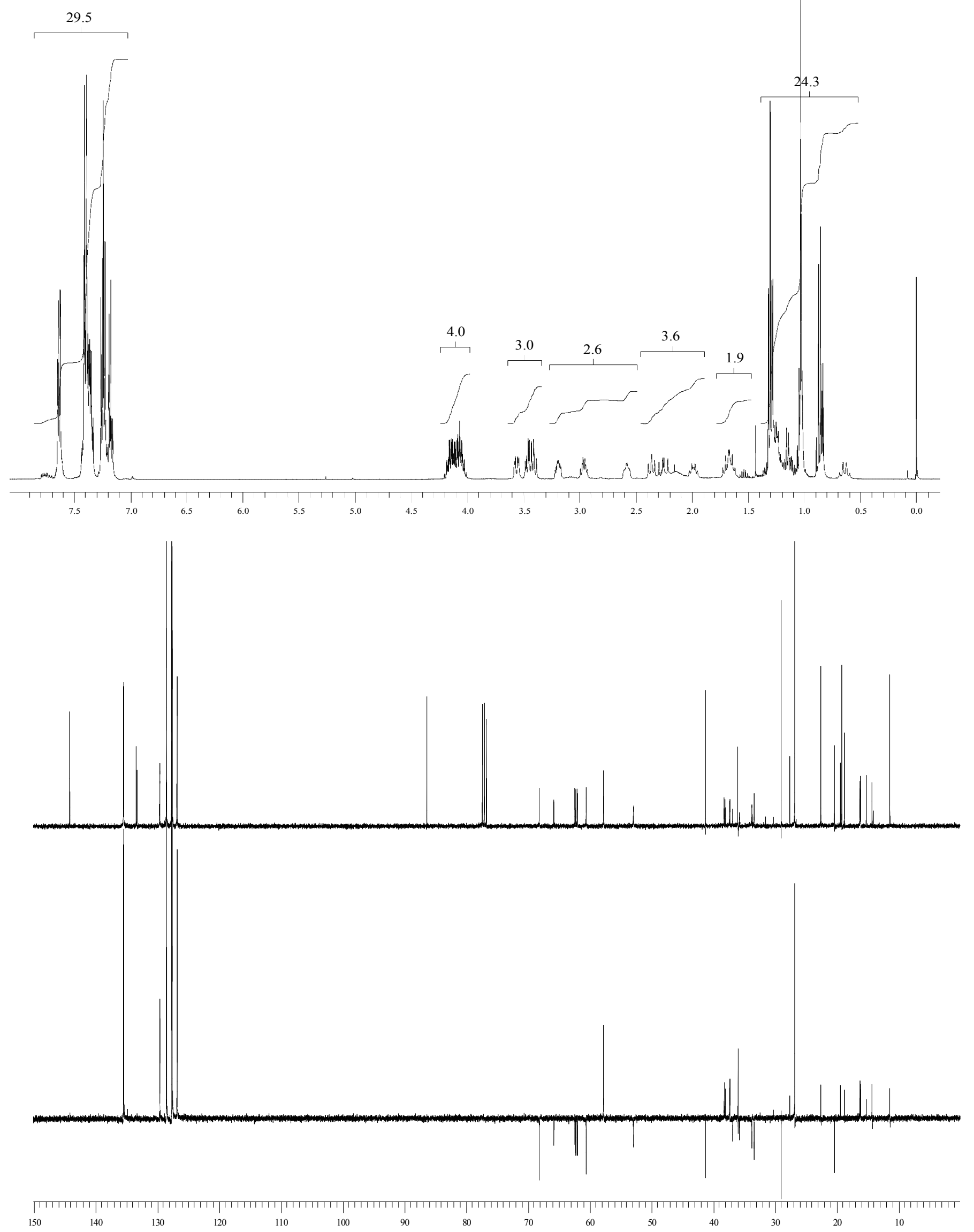

inyl]methylphosphonothioate 19a

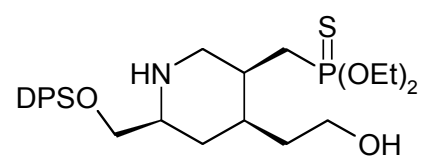

To a solution on trityl ether $\mathbf{1 8 a}(0.28 \mathrm{~g}, 0.34 \mathrm{mmol})$ in $\mathrm{THF} / \mathrm{MeOH}(1: 1,10 \mathrm{~mL})$ was added thiophenol $(0.070 \mathrm{~mL}, 0.69 \mathrm{mmol})$ and $\mathrm{HCl}\left(0.69 \mathrm{~mL}\right.$ of a $1 \mathrm{M}$ aqueous solution). The reaction was heated at $70{ }^{\circ} \mathrm{C}$ for $1 \mathrm{~h}$ then cooled to $\mathrm{rt}$, solid $\mathrm{K}_{2} \mathrm{CO}_{3}$ was added to give a saturated solution that was then extracted with EtOAc. The combined organic phases were dried $\left(\mathrm{MgSO}_{4}\right)$, filtered and evaporated to give the crude product. Purification by flash column chromatography (on silica) using a solvent gradient of $\mathrm{Et}_{2} \mathrm{O} / \mathrm{Et}_{3} \mathrm{~N}$ (99:1) to EtOAc/ $\mathrm{Et}_{3} \mathrm{~N}(99: 1)$ as eluent afforded amino alcohol 19a $(0.11 \mathrm{~g}, 58 \%)$ as a colorless oil. $\mathrm{R}_{\mathrm{f}}$ $0.2\left(\mathrm{Et}_{2} \mathrm{O}\right)$; IR: (neat) $v_{\max } 3610,3055,2931,2860,1471,1427,1390,1259,1230,1203,1111,1078$, 1051, 1028, $957 \mathrm{~cm}^{-1} ;{ }^{1} \mathrm{H}$ NMR (400 MHz, $\left.\mathrm{CDCl}_{3}\right) \delta_{\mathrm{H}}:$ 7.67-7.64 (m, 4H), 7.44-7.35 (m, 6H), 4.22-4.02 (m, 4H), $3.69(\mathrm{t}, J=6.5 \mathrm{~Hz}, 2 \mathrm{H}), 3.60(\mathrm{dd}, J=9.5,3.5 \mathrm{~Hz}, 1 \mathrm{H}), 3.49(\mathrm{dd}, J=9.5,7.0 \mathrm{~Hz}, 1 \mathrm{H}), 3.31$ (dd, $J=14.0,2.0 \mathrm{~Hz}, 1 \mathrm{H}), 2.76-2.70(\mathrm{~m}, 2 \mathrm{H}), 2.49(\mathrm{td}, J=15.0,10.0 \mathrm{~Hz}, 1 \mathrm{H}), 2.17(\mathrm{dd}, J=16.5,10.5 \mathrm{~Hz}$, $1 \mathrm{H}), 1.87-1.68(\mathrm{~m}, 3 \mathrm{H}), 1.58-1.49(\mathrm{~m}, 1 \mathrm{H}), 1.44-1.36(\mathrm{~m}, 1 \mathrm{H}), 1.31(\mathrm{t}, J=7.0 \mathrm{~Hz}, 3 \mathrm{H}), 1.30(\mathrm{t}, J=7.0$ $\mathrm{Hz}, 3 \mathrm{H}), 1.28-1.20(\mathrm{~m}, 2 \mathrm{H}), 1.06(\mathrm{~s}, 9 \mathrm{H}), 0.87(\mathrm{q}, J=12.0 \mathrm{~Hz}, 1 \mathrm{H}) ;{ }^{13} \mathrm{C} \mathrm{NMR}\left(100 \mathrm{MHz}, \mathrm{CDCl}_{3}\right) \delta_{\mathrm{C}}$ : $135.53(\mathrm{CH})$ and $135.47(\mathrm{CH}), 133.5(\mathrm{C}), 133.4(\mathrm{C}), 129.6(\mathrm{CH}), 127.64(\mathrm{CH}), 127.62(\mathrm{CH}), 68.2$ $\left(\mathrm{CH}_{2}\right), 62.25\left(\mathrm{~d}, J_{\mathrm{CP}}=7.0 \mathrm{~Hz}, \mathrm{CH}_{2}\right), 62.22\left(\mathrm{~d}, J_{\mathrm{CP}}=7.0 \mathrm{~Hz}, \mathrm{CH}_{2}\right), 60.2\left(\mathrm{CH}_{2}\right), 58.0(\mathrm{CH}), 50.1\left(\mathrm{CH}_{2}\right)$, $36.5\left(\mathrm{CH}_{2}\right), 36.3\left(\mathrm{~d}, J_{\mathrm{CP}}=14.5 \mathrm{~Hz}, \mathrm{CH}\right), 32.4\left(\mathrm{~d}, J_{\mathrm{CP}}=1.5 \mathrm{~Hz}, \mathrm{CH}\right), 30.2\left(\mathrm{CH}_{2}\right), 29.4\left(\mathrm{~d}, J_{\mathrm{CP}}=110.0 \mathrm{~Hz}\right.$, $\left.\mathrm{CH}_{2}\right), 26.8\left(\mathrm{CH}_{3}\right), 19.2(\mathrm{C}), 16.2\left(\mathrm{~d}, J_{\mathrm{CP}}=7.0, \mathrm{CH}_{3}\right) ; \mathrm{LRMS}\left(\mathrm{CI}, \mathrm{NH}_{3}\right) m / z 564\left([\mathrm{M}+\mathrm{H}]^{+}, 75 \%\right), 546$ (100), 196 (93); HRMS (CI, $\mathrm{NH}_{3}$ ) m/z [M+H $\mathrm{H}^{+}$found 564.2727; calcd 564.2733 for $\mathrm{C}_{29} \mathrm{H}_{46} \mathrm{NO}_{4} \mathrm{PSSi}$. 


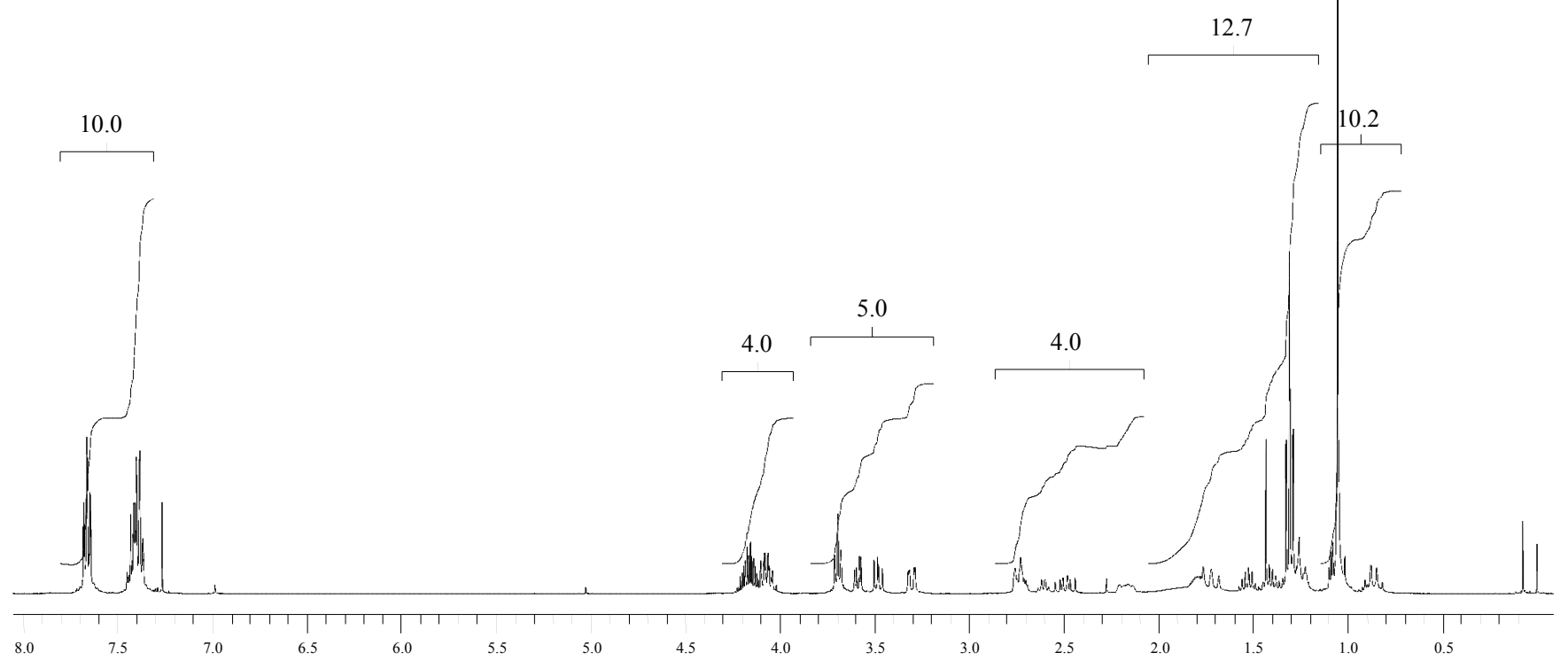




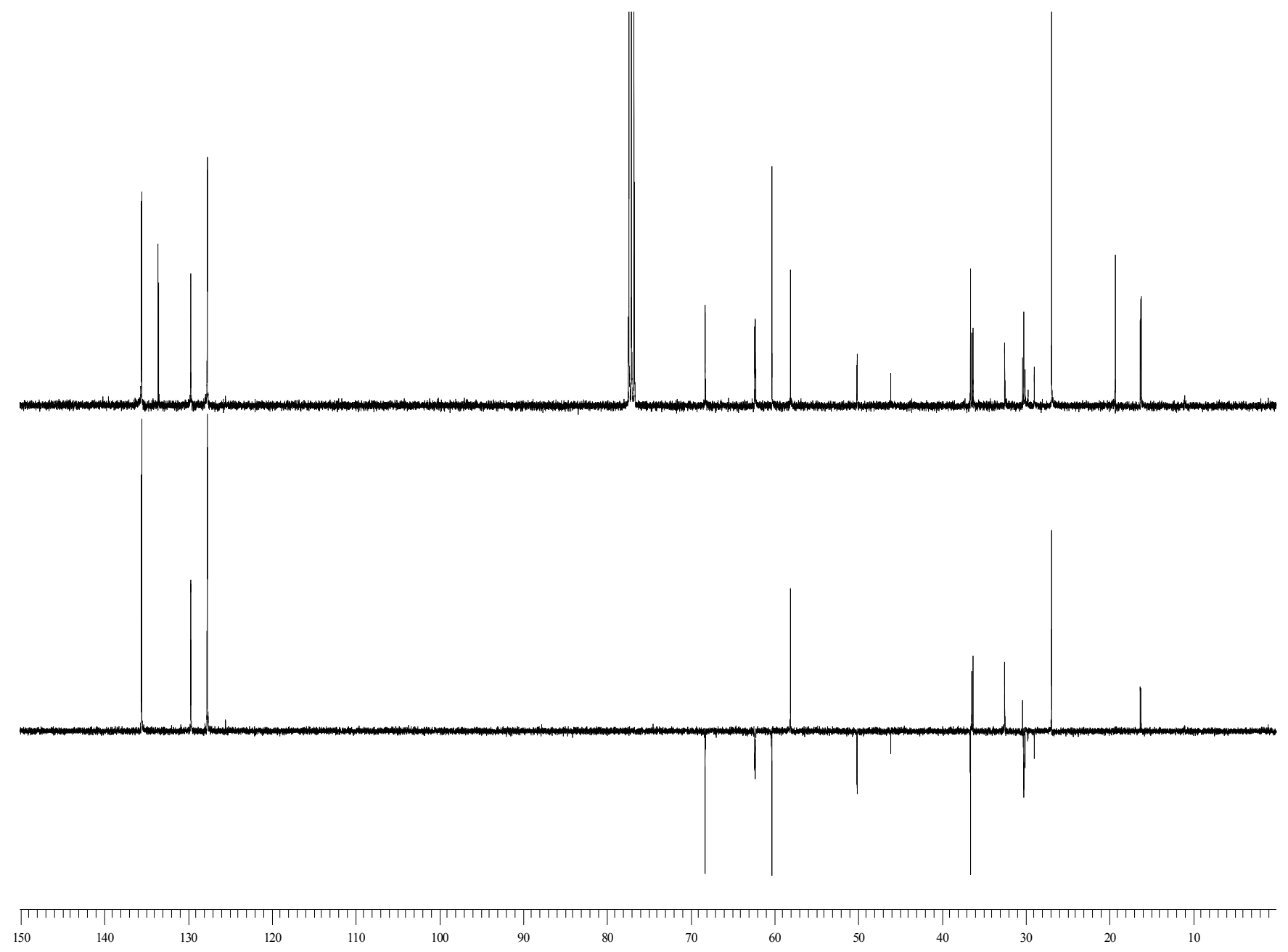

$O, O$-Diethyl $[$ rel-(3R,4R,6S)-6-(\{[tert-butyl(diphenyl)silyl]oxy\}methyl)-4-(2-hydroxyethyl)piperidinyl]methylphosphonothioate 19b<smiles>CCOCCCC1CC(COCCO)CNC1CCO</smiles>

To a solution on trityl ether $\mathbf{1 8 b}(0.25 \mathrm{~g}, 0.31 \mathrm{mmol})$ in $\mathrm{THF} / \mathrm{MeOH}(1: 1,10 \mathrm{~mL})$ was added thiophenol $(0.064 \mathrm{~mL}, 0.62 \mathrm{mmol})$ and $\mathrm{HCl}\left(0.62 \mathrm{~mL}\right.$ of a $1 \mathrm{M}$ aqueous solution). The reaction was heated at $70{ }^{\circ} \mathrm{C}$ for $1 \mathrm{~h}$ then cooled to $\mathrm{rt}$, solid $\mathrm{K}_{2} \mathrm{CO}_{3}$ was added to give a saturated solution that was then extracted with EtOAc. The combined organic phases were dried $\left(\mathrm{MgSO}_{4}\right)$, filtered and evaporated to give the crude product. Purification by flash column chromatography (on silica) using $\mathrm{Et}_{2} \mathrm{O} / \mathrm{Et}_{3} \mathrm{~N}$ (99:1) then EtOAc/Et $t_{3} \mathrm{~N}(99: 1)$ as eluent afforded amino alcohol $19 b(0.14 \mathrm{~g}, 80 \%)$ as a colorless oil. $\mathrm{R}_{\mathrm{f}} 0.5$ (EtOAc); IR: (neat) $v_{\max }$ 3616, 3052, 2956, 2931, 2858, 1471, 1427, 1390, 1280, 1232, 1203, 1111, 
1051, 1026, 957, $908 \mathrm{~cm}^{-1} ;{ }^{1} \mathrm{H}$ NMR (400 MHz, $\left.\mathrm{CDCl}_{3}\right) \delta_{\mathrm{H}}: 7.67-7.63(\mathrm{~m}, 4 \mathrm{H}), 7.45-7.35(\mathrm{~m}, 6 \mathrm{H}), 4.21-$ $4.03(\mathrm{~m}, 4 \mathrm{H}), 3.72-3.67(\mathrm{~m}, 1 \mathrm{H}), 3.63-3.51(\mathrm{~m}, 4 \mathrm{H}), 2.75-2.69(\mathrm{~m}, 1 \mathrm{H}), 2.41(\mathrm{t}, J=11.0 \mathrm{~Hz}, 1 \mathrm{H}), 2.21$ (dd, $J=18.0,12.0 \mathrm{~Hz}, 1 \mathrm{H}), 2.06(\mathrm{br} \mathrm{s}, 1 \mathrm{H}), 1.89(\mathrm{dt}, J=12.0,7.5 \mathrm{~Hz}, 1 \mathrm{H}), 1.80-1.59(\mathrm{~m}, 3 \mathrm{H}), 1.38-1.21$ (m, 9H), $1.05(\mathrm{~s}, 9 \mathrm{H}), 0.89(\mathrm{q}, J=11.5,1 \mathrm{H}) ;{ }^{13} \mathrm{C} \mathrm{NMR}\left(100 \mathrm{MHz}, \mathrm{CDCl}_{3}\right) \delta_{\mathrm{C}}: 135.55(\mathrm{CH}), 135.48$ $(\mathrm{CH}), 133.8(\mathrm{C}), 133.0(\mathrm{C}), 129.8(\mathrm{CH}), 127.7(\mathrm{CH}), 67.1\left(\mathrm{CH}_{2}\right), 62.6\left(\mathrm{~d}, J_{\mathrm{CP}}=7.0 \mathrm{~Hz}, \mathrm{CH}_{2}\right), 62.3(\mathrm{~d}$, $\left.J_{\mathrm{CP}}=7.0 \mathrm{~Hz}, \mathrm{CH}_{2}\right), 59.8\left(\mathrm{CH}_{2}\right), 57.9(\mathrm{CH}), 51.9\left(\mathrm{CH}_{2}\right), 37.3\left(\mathrm{~d}, J_{\mathrm{CP}}=16.0 \mathrm{~Hz}, \mathrm{CH}\right), 35.9\left(\mathrm{~d}, J_{\mathrm{CP}}=111.0\right.$ $\left.\mathrm{Hz}, \mathrm{CH}_{2}\right), 35.6\left(\mathrm{CH}_{2}\right), 30.3(\mathrm{CH}), 29.7\left(\mathrm{CH}_{2}\right), 26.9\left(\mathrm{CH}_{3}\right), 19.2(\mathrm{C}), 16.25\left(\mathrm{~d}, J_{\mathrm{CP}}=7.5 \mathrm{~Hz}, \mathrm{CH}_{3}\right), 16.21$ $\left(\mathrm{d}, J_{\mathrm{CP}}=7.5 \mathrm{~Hz}, \mathrm{CH}_{3}\right) ; \mathrm{LRMS}\left(\mathrm{CI}, \mathrm{NH}_{3}\right) \mathrm{m} / z, 564\left([\mathrm{M}+\mathrm{H}]^{+}, 50 \%\right), 546(75), 196(100) ; \mathrm{HRMS}\left(\mathrm{CI}, \mathrm{NH}_{3}\right)$ $m / z\left[\mathrm{M}+\mathrm{H}^{+}\right.$found 564.2734; calcd 564.2733 for $\mathrm{C}_{29} \mathrm{H}_{46} \mathrm{NO}_{4} \mathrm{PSSi}$.

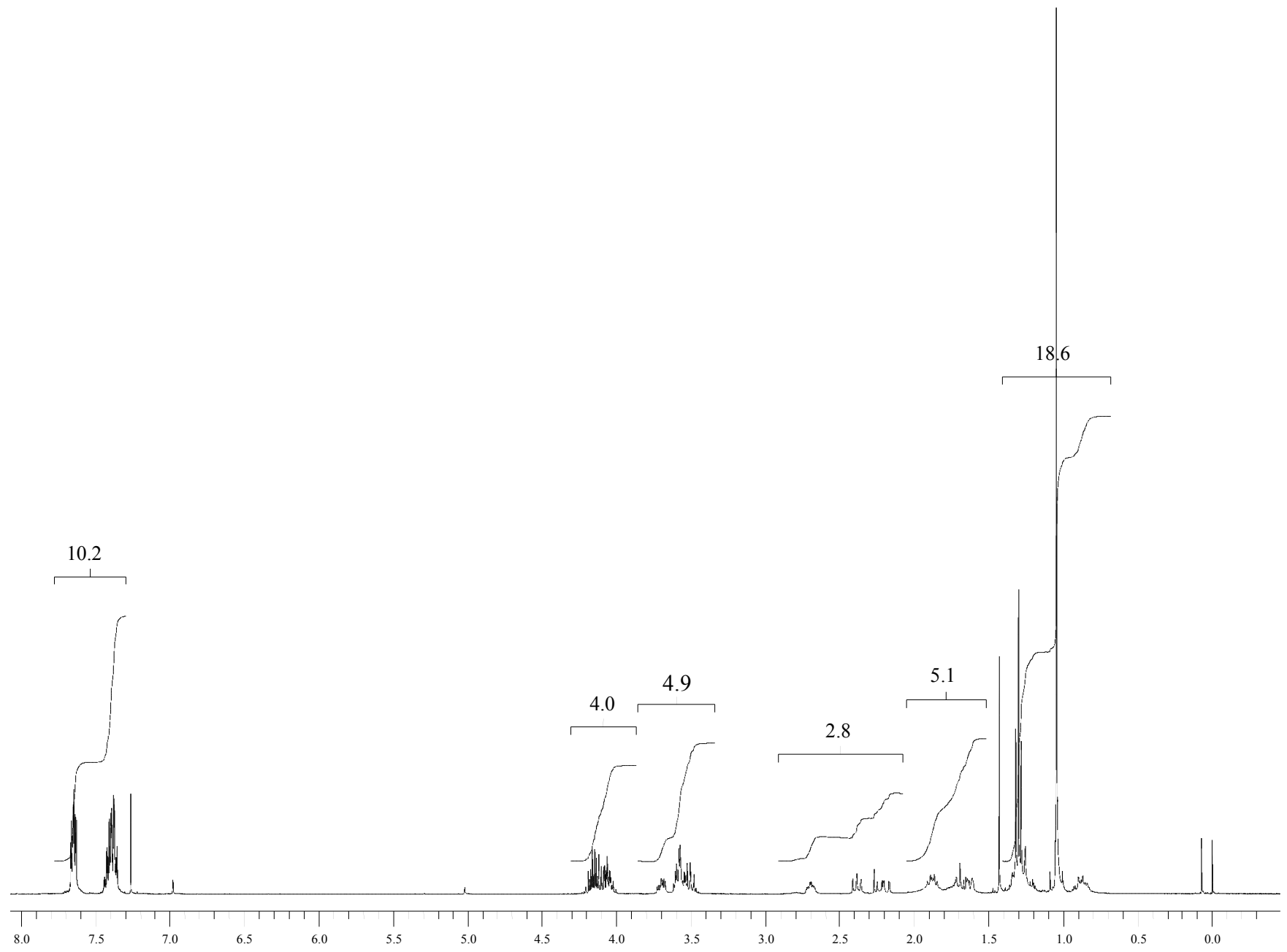




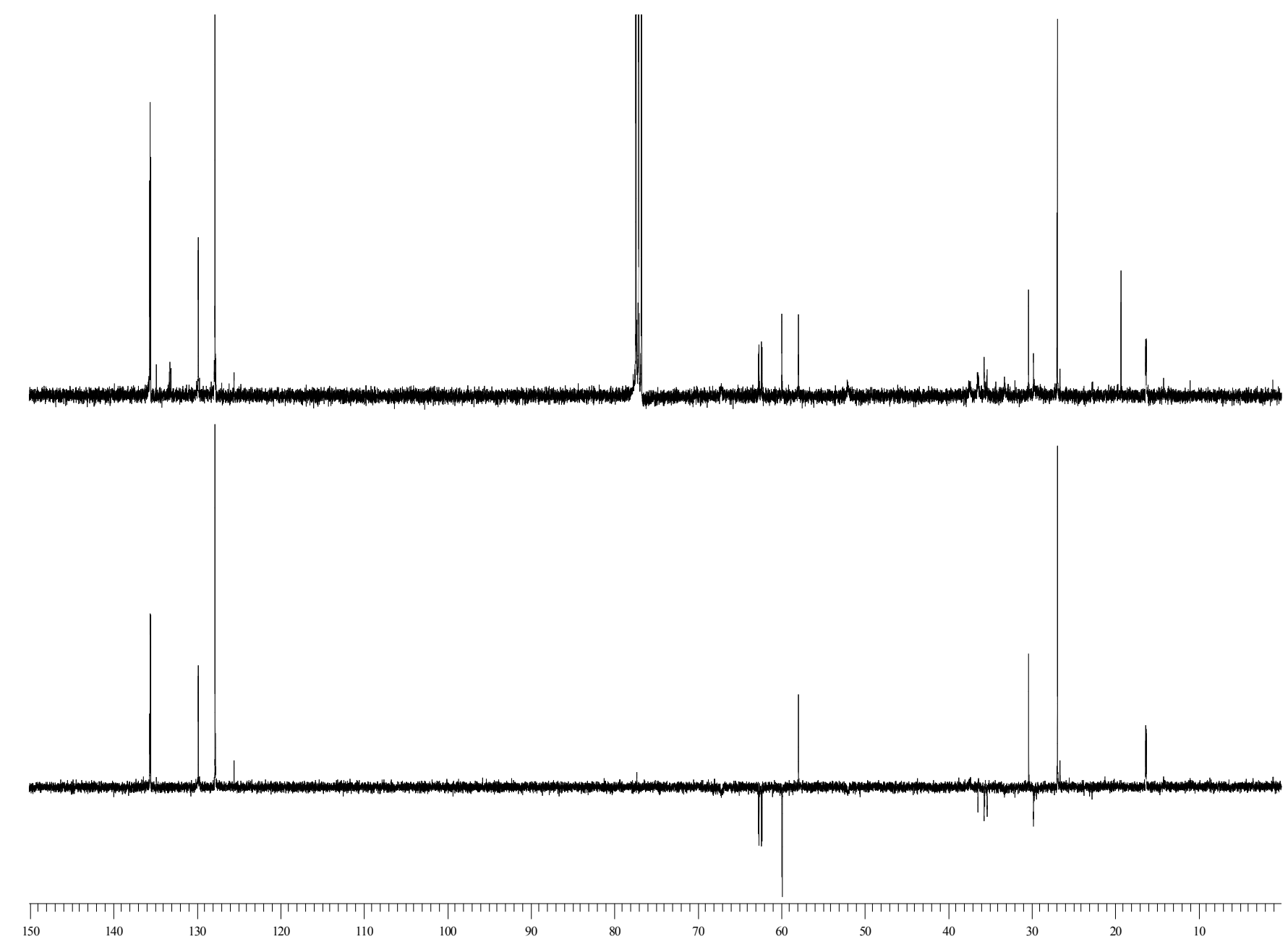

$O, O$-Diethyl [rel-(3S,4R,6S)-6-(\{[tert-butyl(diphenyl)silyl]oxy $\}$ methyl)-1-azabicyclo[2.2.2]-oct-3-yl]methylphosphonothioate 20a

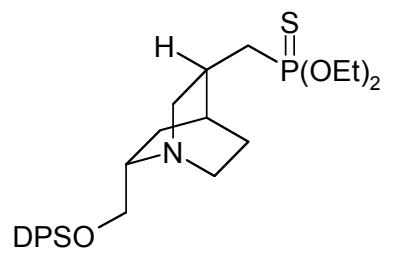

Amino alcohol 19a $(0.11 \mathrm{~g}, 0.20 \mathrm{mmol})$ was dissolved in anhydrous $\mathrm{CH}_{2} \mathrm{Cl}_{2}(10 \mathrm{~mL})$ and cooled to $0{ }^{\circ} \mathrm{C}$. A solution of $\mathrm{MsCl}\left(1.03 \mathrm{~mL}\right.$ of a $0.14 \mathrm{M}$ solution in $\left.\mathrm{CH}_{2} \mathrm{Cl}_{2}, 0.14 \mathrm{mmol}\right)$ was added slowly over $0.1 \mathrm{~h}$ then pyridine $(0.11 \mathrm{~mL}, 1.38 \mathrm{mmol})$ was added and the yellow solution was stirred at $\mathrm{rt}$ for $16 \mathrm{~h}$. After this time $\mathrm{Et}_{2} \mathrm{O}$ was added and the organic phase was washed with $\mathrm{CuSO}_{4}$ (saturated aqueous solution) and then once with $\mathrm{NaHCO}_{3}$ (saturated aqueous solution). The organic phase was dried $\left(\mathrm{MgSO}_{4}\right)$, 
filtered and evaporated to give the crude product that was dissolved in acetonitrile $(50 \mathrm{~mL})$. The reaction mixture was heated to reflux for $3 \mathrm{~h}$ after which time the reaction was complete (as judged by TLC). The solvent was removed in vacuo and the residue was dissolved in EtOAc. The organic phase was washed with $\mathrm{NaHCO}_{3}$ (saturated aqueous solution), then with brine and then dried $\left(\mathrm{MgSO}_{4}\right)$, filtered and evaporated. Purification by flash column chromatography (on silica) using a solvent gradient of petroleum ether/ $\mathrm{Et}_{2} \mathrm{O} / \mathrm{Et}_{3} \mathrm{~N}(75: 25: 1)$ to $\mathrm{Et}_{2} \mathrm{O} / \mathrm{Et}_{3} \mathrm{~N}(99: 1)$ as eluent afforded quinuclidine 20a (72 mg, 92\%). $\mathrm{R}_{\mathrm{f}} 0.4\left(\mathrm{Et}_{2} \mathrm{O}\right)$; IR: $\left(\mathrm{CH}_{2} \mathrm{Cl}_{2}\right) v_{\max } 2933,2860,1604,1471,1429,1390,1277,1113,1047$, 1027, $957 \mathrm{~cm}^{-1} ;{ }^{1} \mathrm{H}$ NMR (400 MHz, $\left.\mathrm{CDCl}_{3}\right) \delta_{\mathrm{H}}: 7.70-7.65(\mathrm{~m}, 4 \mathrm{H}), 7.44-7.35(\mathrm{~m}, 6 \mathrm{H}), 4.21-4.01(\mathrm{~m}$, 4H), $3.75(\mathrm{dd}, J=10.5,6.0 \mathrm{~Hz}, 1 \mathrm{H}), 3.71(\mathrm{dd}, J=10.5,6.0 \mathrm{~Hz}, 1 \mathrm{H}), 3.24(\mathrm{ddd}, J=13.5,9.5,2.0 \mathrm{~Hz}$, $1 \mathrm{H}), 3.03-2.91(\mathrm{~m}, 2 \mathrm{H}), 2.76-2.62(\mathrm{~m}, 1 \mathrm{H}), 2.48(\mathrm{dd}, J=13.5,7.0 \mathrm{~Hz}, 1 \mathrm{H}), 2.27-2.17(\mathrm{~m}, 1 \mathrm{H}), 2.15-$ $1.96(\mathrm{~m}, 2 \mathrm{H}), 1.85-1.74(\mathrm{~m}, 2 \mathrm{H}), 1.61-1.47(\mathrm{~m}, 2 \mathrm{H}), 1.31(\mathrm{t}, J=7.0 \mathrm{~Hz}, 3 \mathrm{H}), 1.30(\mathrm{t}, J=7.0 \mathrm{~Hz}, 3 \mathrm{H})$, 1.32-1.27 (m, 1H), ${ }^{\dagger} 1.06(\mathrm{~s}, 9 \mathrm{H}) ;{ }^{13} \mathrm{C} \mathrm{NMR}\left(100 \mathrm{MHz}, \mathrm{CDCl}_{3}\right) \delta_{\mathrm{C}}: 135.6(\mathrm{CH}), 133.6(\mathrm{C}), 129.6(\mathrm{CH})$, $127.6(\mathrm{CH}), 66.4\left(\mathrm{CH}_{2}\right), 62.3\left(\mathrm{~d}, J_{\mathrm{CP}}=6.0 \mathrm{~Hz}, \mathrm{CH}_{2}\right), 57.7\left(\mathrm{CH}_{2}\right), 56.5(\mathrm{CH}), 42.9\left(\mathrm{CH}_{2}\right), 39.2\left(\mathrm{~d}, J_{\mathrm{CP}}=\right.$ $\left.111.0 \mathrm{~Hz}, \mathrm{CH}_{2}\right), 31.1\left(\mathrm{CH}_{2}\right), 30.2\left(\mathrm{~d}, J_{\mathrm{CP}}=4.0 \mathrm{~Hz}, \mathrm{CH}\right), 28.0\left(\mathrm{~d}, J_{\mathrm{CP}}=13.0 \mathrm{~Hz}, \mathrm{CH}\right), 26.9\left(\mathrm{CH}_{3}\right), 21.1$ $\left(\mathrm{CH}_{2}\right), 19.3(\mathrm{C}), 16.2\left(\mathrm{~d}, J_{\mathrm{CP}}=7.5 \mathrm{~Hz}, \mathrm{CH}_{3}\right) ; \mathrm{LRMS}\left(\mathrm{CI}, \mathrm{NH}_{3}\right) \mathrm{m} / z 546\left([\mathrm{M}+\mathrm{H}]^{+}, 100 \%\right) ; \mathrm{HRMS}(\mathrm{CI}$, $\left.\mathrm{NH}_{3}\right) m / z\left[\mathrm{M}+\mathrm{H}^{+}\right]$found 546.2624; calcd 546.2627 for $\mathrm{C}_{29} \mathrm{H}_{44} \mathrm{NO}_{3}$ PSSi.

\footnotetext{
' Observed from the HSQC spectrum with a correlation to the $\mathrm{CH}_{2}$ signal at $21.1 \mathrm{ppm}$.
} 


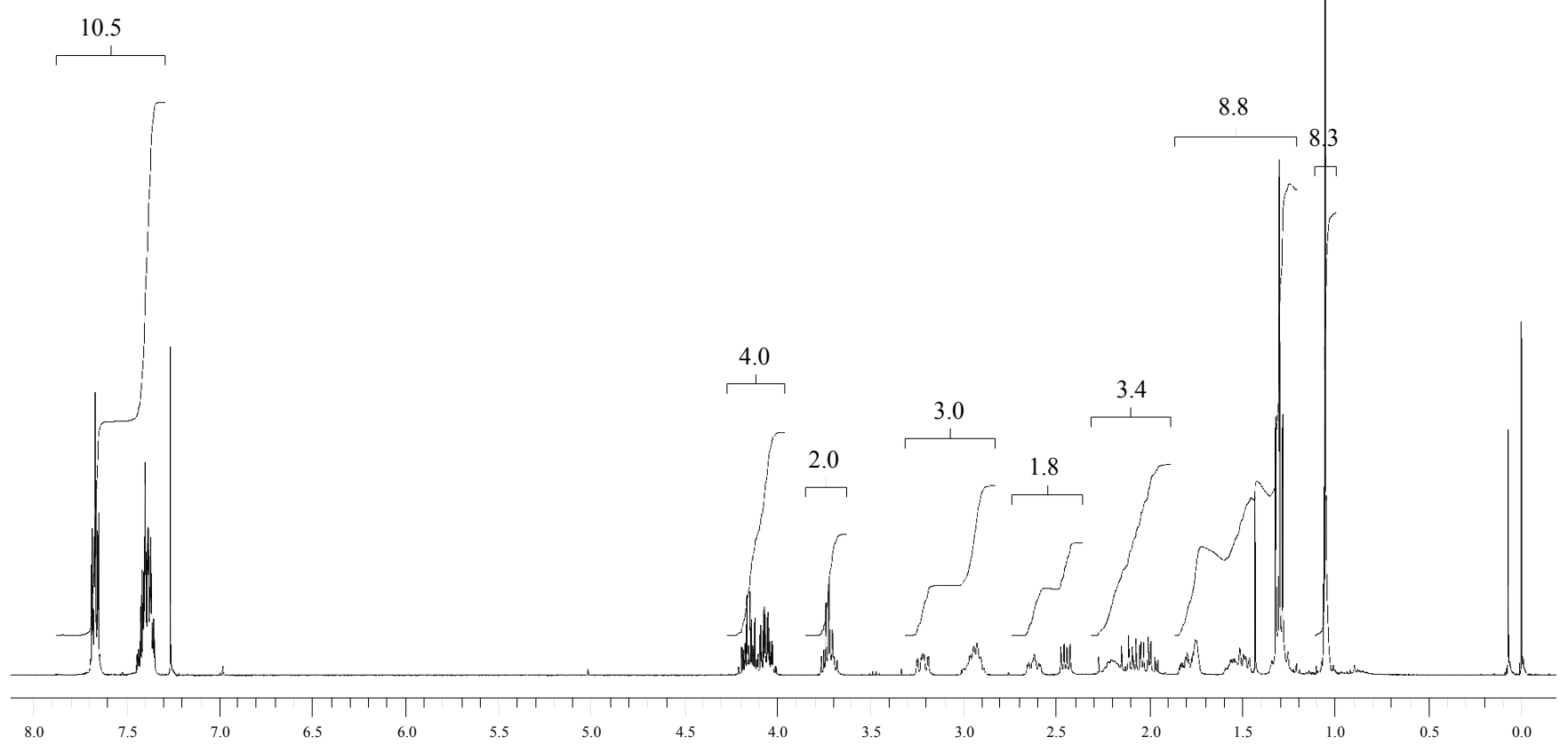




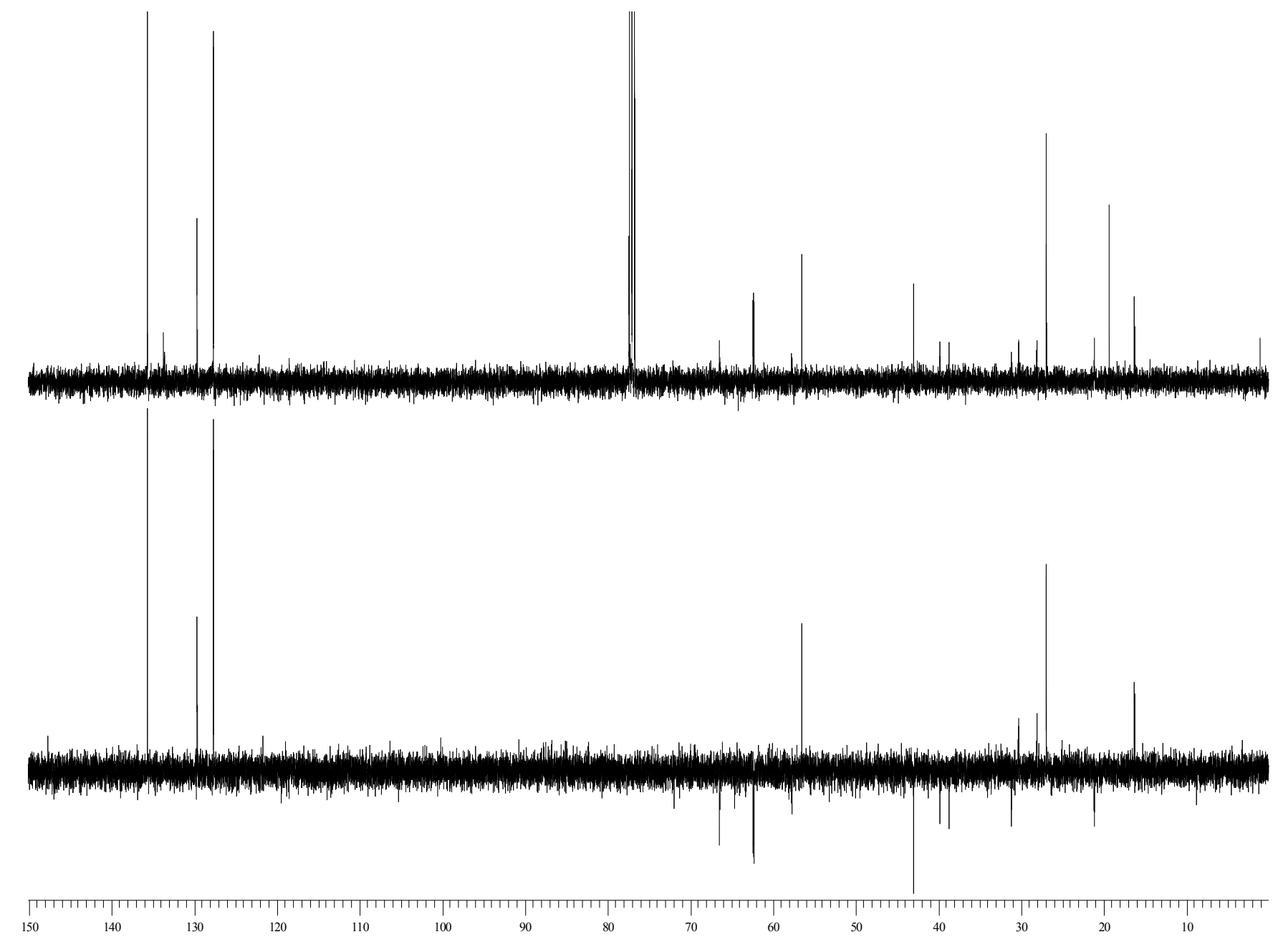

$O, O$-Diethyl $\quad[$ rel-(3R,4R,6S)-6-(\{[tert-butyl(diphenyl)silyl $]$ oxy $\}$ methyl)-1-azabicyclo-[2.2.2]oct-3-

yl]methylphosphonothioate 20b

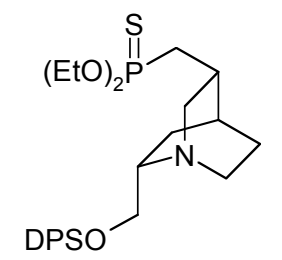

Amino alcohol 19b $(0.14 \mathrm{~g}, 0.24 \mathrm{mmol})$ was dissolved in anhydrous $\mathrm{CH}_{2} \mathrm{Cl}_{2}(10 \mathrm{~mL})$ and cooled to 0 ${ }^{\circ} \mathrm{C}$. A solution of $\mathrm{MsCl}\left(1.30 \mathrm{~mL}\right.$ of a $0.14 \mathrm{M}$ solution in $\left.\mathrm{CH}_{2} \mathrm{Cl}_{2}, 0.18 \mathrm{mmol}\right)$ was added slowly over $0.1 \mathrm{~h}$ then pyridine $(0.14 \mathrm{~mL}, 1.73 \mathrm{mmol})$ was added and the yellow solution was stirred at $\mathrm{rt}$ for $16 \mathrm{~h}$. After this time $\mathrm{Et}_{2} \mathrm{O}$ was added and the organic phase was washed with $\mathrm{CuSO}_{4}$ (saturated aqueous solution) and then once with $\mathrm{NaHCO}_{3}$ (saturated aqueous solution). The organic phase was dried $\left(\mathrm{MgSO}_{4}\right)$, filtered and evaporated to give the crude product that was dissolved in acetonitrile $(50 \mathrm{~mL})$. 
The reaction mixture was heated to reflux for $3 \mathrm{~h}$ after which time the reaction was complete (as judged by TLC). The solvent was removed in vacuo, then the residue was dissolved in EtOAc and the organic phase was washed with $\mathrm{NaHCO}_{3}$ and then with brine. The organic phase was then dried $\left(\mathrm{MgSO}_{4}\right)$, filtered and evaporated. Purification by flash column chromatography (on silica) using a solvent gradient of petroleum ether/ $/ \mathrm{Et}_{2} \mathrm{O} / \mathrm{Et}_{3} \mathrm{~N}(75: 25: 1)$ to $\mathrm{Et}_{2} \mathrm{O} / \mathrm{Et}_{3} \mathrm{~N}$ (99:1) as eluent afforded quinuclidine 20b (61 mg, 62\%). $\mathrm{R}_{\mathrm{f}} 0.4\left(\mathrm{Et}_{2} \mathrm{O}\right)$; IR: $\left(\mathrm{CH}_{2} \mathrm{Cl}_{2}\right) v_{\max } 2933,2864,1471,1271,1113,1047,1026,956 \mathrm{~cm}^{-1} ;{ }^{1} \mathrm{H}$ NMR $\left(400 \mathrm{MHz}, \mathrm{CDCl}_{3}\right) \delta_{\mathrm{H}}: 7.69-7.65(\mathrm{~m}, 4 \mathrm{H}), 7.43-7.35(\mathrm{~m}, 6 \mathrm{H}), 4.21-4.01(\mathrm{~m}, 4 \mathrm{H}), 3.74(\mathrm{dd}, J=$ 10.0, $5.5 \mathrm{~Hz}, 1 \mathrm{H}), 3.68(\mathrm{dd}, J=10.0,7.0 \mathrm{~Hz}, 1 \mathrm{H}), 3.18(\mathrm{dd}, J=13.5,9.5 \mathrm{~Hz}, 1 \mathrm{H}), 3.00-2.80(\mathrm{~m}, 2 \mathrm{H})$, 2.63-2.56 (m, 2H), 2.19-2.04 (m, 3H), 1.83-1.74 (m, 2H), 1.53-1.45 (m, 2H), 1.37-1.31 (m, 1H), $1.31(\mathrm{t}$, $J=7.0 \mathrm{~Hz}, 6 \mathrm{H}), 1.06(\mathrm{~s}, 9 \mathrm{H}) ;{ }^{13} \mathrm{C} \mathrm{NMR}\left(100 \mathrm{MHz}, \mathrm{CDCl}_{3}\right) \delta_{\mathrm{c}}: 135.6(\mathrm{CH}), 133.6(\mathrm{C}), 129.6(\mathrm{CH})$, 127.6 (CH), $66.8\left(\mathrm{CH}_{2}\right), 62.3,\left(\mathrm{~d}, J_{\mathrm{CP}}=7.5 \mathrm{~Hz}, \mathrm{CH}_{2}\right), 62.2\left(\mathrm{~d}, J_{\mathrm{CP}}=7.0 \mathrm{~Hz}, \mathrm{CH}_{2}\right), 58.0\left(\mathrm{CH}_{2}\right), 57.1(\mathrm{CH})$, $42.0\left(\mathrm{CH}_{2}\right), 40.3\left(\mathrm{~d}, J_{\mathrm{CP}}=111.0 \mathrm{~Hz}, \mathrm{CH}_{2}\right), 30.5\left(\mathrm{~d}, J_{\mathrm{CP}}=3.0 \mathrm{~Hz}, \mathrm{CH}\right), 28.1\left(\mathrm{CH}_{2}\right), 27.4\left(\mathrm{~d}, J_{\mathrm{CP}}=13.5 \mathrm{~Hz}\right.$, $\mathrm{CH}), 26.9\left(\mathrm{CH}_{3}\right), 24.7\left(\mathrm{CH}_{2}\right), 19.2(\mathrm{C}), 16.22\left(\mathrm{~d}, J_{\mathrm{CP}}=7.0 \mathrm{~Hz}, \mathrm{CH}_{3}\right), 16.20\left(\mathrm{~d}, J_{\mathrm{CP}}=7.0 \mathrm{~Hz}, \mathrm{CH}_{3}\right)$; LRMS $\left(\mathrm{CI}, \mathrm{NH}_{3}\right) m / z 546\left([\mathrm{M}+\mathrm{H}]^{+}, 100 \%\right)$; HRMS $\left(\mathrm{CI}, \mathrm{NH}_{3}\right) \mathrm{m} / z\left[\mathrm{M}+\mathrm{H}^{+}\right]$found 546.2618; calcd 546.2627 for $\mathrm{C}_{29} \mathrm{H}_{44} \mathrm{NO}_{3} \mathrm{PSSi}$. 

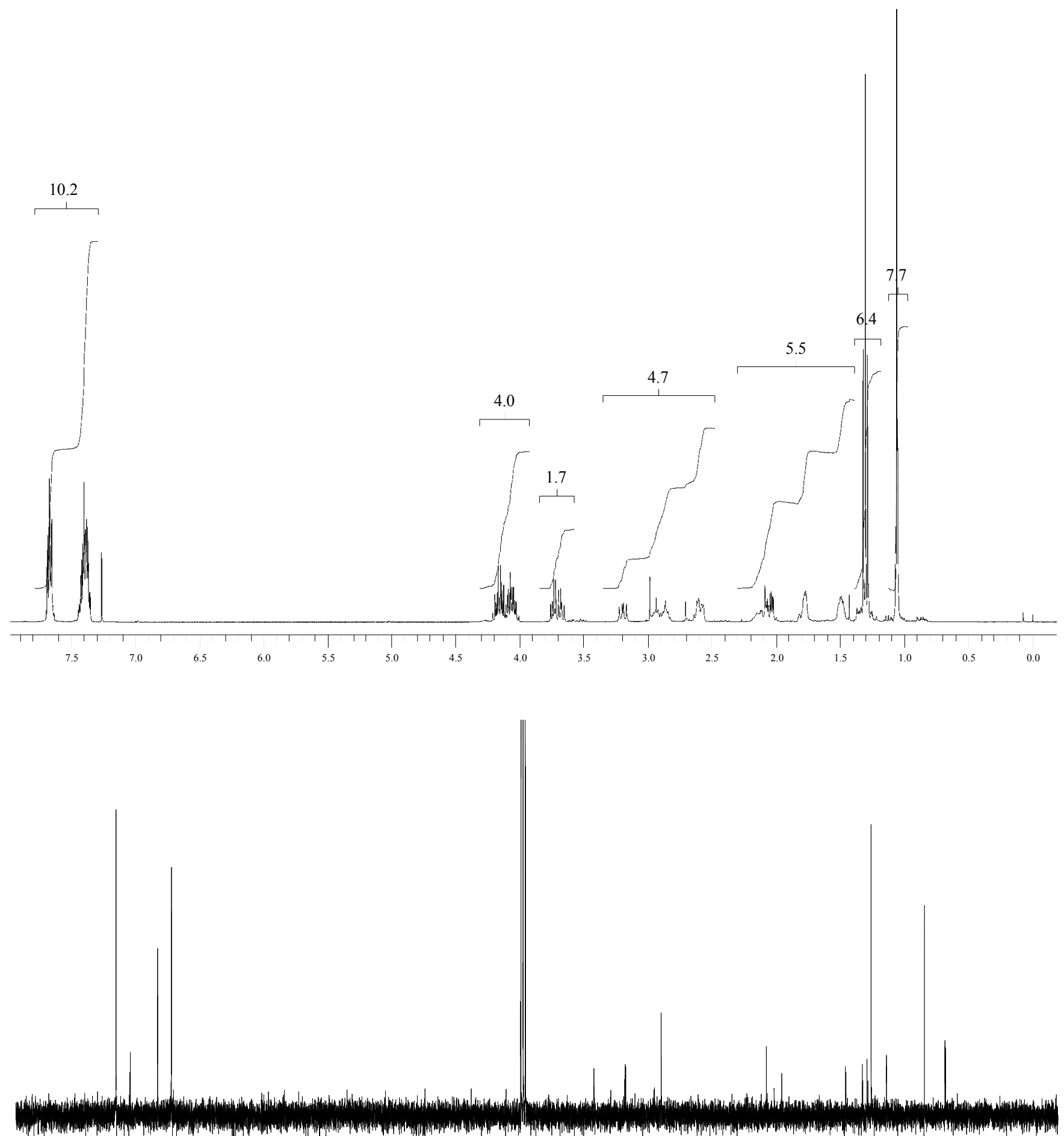

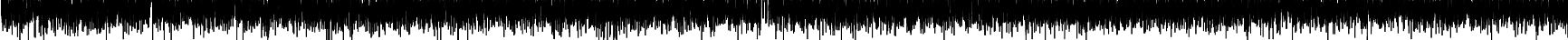
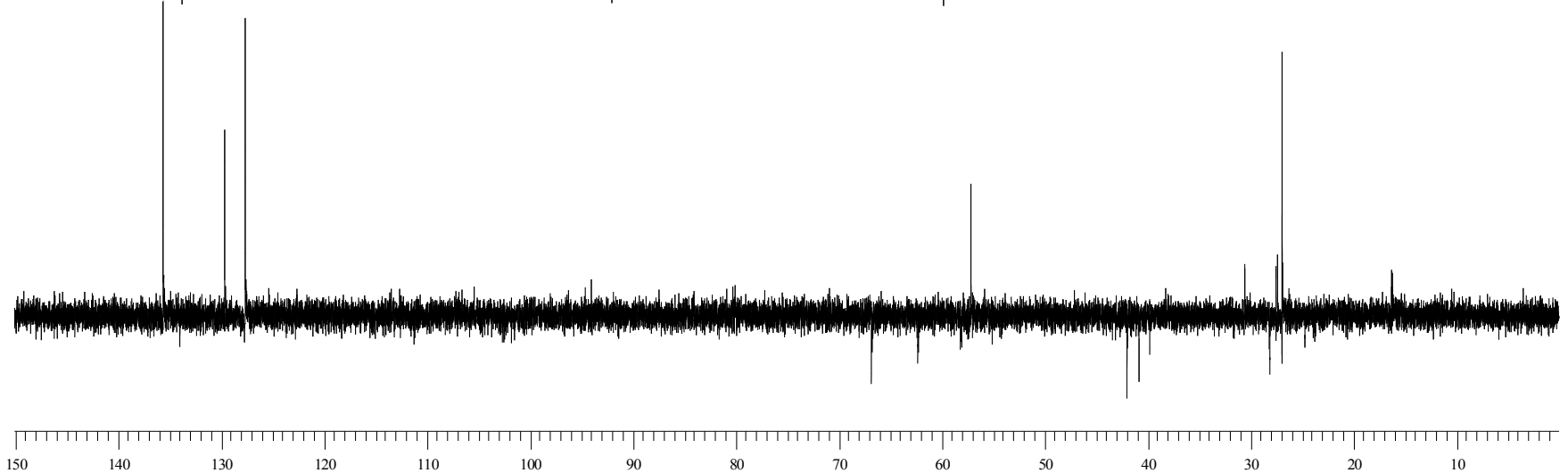
Full NMR assignment of $\quad r e l-(2 S, 4 R, 5 R)-2-(\{[$ tert-Butyl(diphenyl)silyl $]$ oxy $\}$ methyl)-5-(2,2diphenylvinyl)-1-azabicyclo[2.2.2]octane 21a

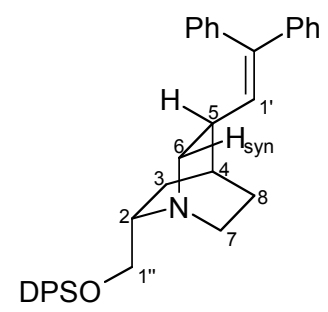

${ }^{1} \mathrm{H}$ NMR (400 MHz, $\mathrm{CDCl}_{3}$ ) $\delta_{\mathrm{H}}:$ 7.60-7.63 (m, 4H, Ar), 7.41-7.32 (m, 9H, Ar), 7.27-7.20 (m, 5H, Ar), 7.15-7.13 (m, 2H, Ar), $6.20\left(\mathrm{~d}, J=10.0 \mathrm{~Hz}, 1 \mathrm{H}, \mathrm{Ph}_{2} \mathrm{C}=\mathrm{C} \underline{H}\right), 3.71\left(\mathrm{~d}, J=6.0 \mathrm{~Hz}, 2 \mathrm{H}, \underline{\mathrm{H}}_{2} \mathrm{OSi}\right), 3.11-$ $3.00\left(\mathrm{~m}, 2 \mathrm{H}, \mathrm{H}-6_{\mathrm{anti}}, \mathrm{H}-7_{\mathrm{ax}}\right), 2.85-2.74\left(\mathrm{~m}, 2 \mathrm{H}, \mathrm{H}-2, \mathrm{H}-7_{\mathrm{ax}}\right), 2.62\left(\mathrm{dd}, J=13.5,7.0 \mathrm{~Hz}, 1 \mathrm{H}, \mathrm{H}-6_{\mathrm{syn}}\right), 2.50-$ $2.43(\mathrm{~m}, 1 \mathrm{H}, \mathrm{H}-5), 1.93-1.81\left(\mathrm{~m}, 1 \mathrm{H}, \mathrm{H}-8_{\text {eq }}\right), 1.80-1.75(\mathrm{~m}, 1 \mathrm{H}, \mathrm{H}-4), 1.65-1.57\left(\mathrm{~m}, 1 \mathrm{H}, \mathrm{H}-3_{\text {eq }}\right), 1.45-$ $1.33\left(\mathrm{~m}, 2 \mathrm{H}, \mathrm{H}-3_{\mathrm{ax}}, \mathrm{H}-8_{\mathrm{ax}}\right), 1.04\left(\mathrm{~s}, 9 \mathrm{H}, \mathrm{SiC}\left(\mathrm{C}_{3}\right)_{3}\right) ;{ }^{13} \mathrm{C} \mathrm{NMR}\left(100 \mathrm{MHz}, \mathrm{CDCl}_{3}\right) \delta_{\mathrm{C}}: 142.6,142.4$ and $140.1\left(\mathrm{Ph}_{2} \underline{C}=\mathrm{CH}\right.$ and $\left.2 \times \underline{C}_{\mathrm{ipso}}, \mathrm{Ar}\right), 135.6\left(4 \times \underline{C}_{\text {meta }}, \mathrm{Ph}_{2} \mathrm{Si}, \mathrm{Ar}\right), 133.5$ and $133.4\left(2 \times \underline{C}_{\mathrm{ipss}}, \mathrm{Ph}_{2} \mathrm{Si}, \mathrm{Ar}\right)$, $131.7\left(\mathrm{Ph}_{2} \mathrm{C}=\underline{C} \mathrm{H}\right), 129.7,129.6,128.2,128.1,127.62,127.59,127.3,127.1$ and $127.0(16 \times \underline{C} \mathrm{H}, \mathrm{Ar})$, $66.2\left(\underline{\mathrm{C}} \mathrm{H}_{2} \mathrm{OSi}\right), 56.7\left(\underline{\mathrm{C}} \mathrm{H}_{2}, \mathrm{C}-6\right), 56.4(\underline{\mathrm{CH}}, \mathrm{C}-2), 43.1\left(\underline{\mathrm{C}} \mathrm{H}_{2}, \mathrm{C}-7\right), 35.4(\underline{\mathrm{C}} \mathrm{H}, \mathrm{C}-5), 30.2\left(\underline{\mathrm{C}} \mathrm{H}_{2}, \mathrm{C}-3\right), 28.4$ $(\underline{C} \mathrm{H}, \mathrm{C}-4), 26.8\left(\mathrm{SiC}\left(\underline{C H}_{3}\right)_{3}\right), 21.5\left(\underline{\mathrm{C}} \mathrm{H}_{2}, \mathrm{C}-8\right), 19.2\left(\mathrm{Si} \underline{C}\left(\mathrm{CH}_{3}\right)_{3}\right)$.

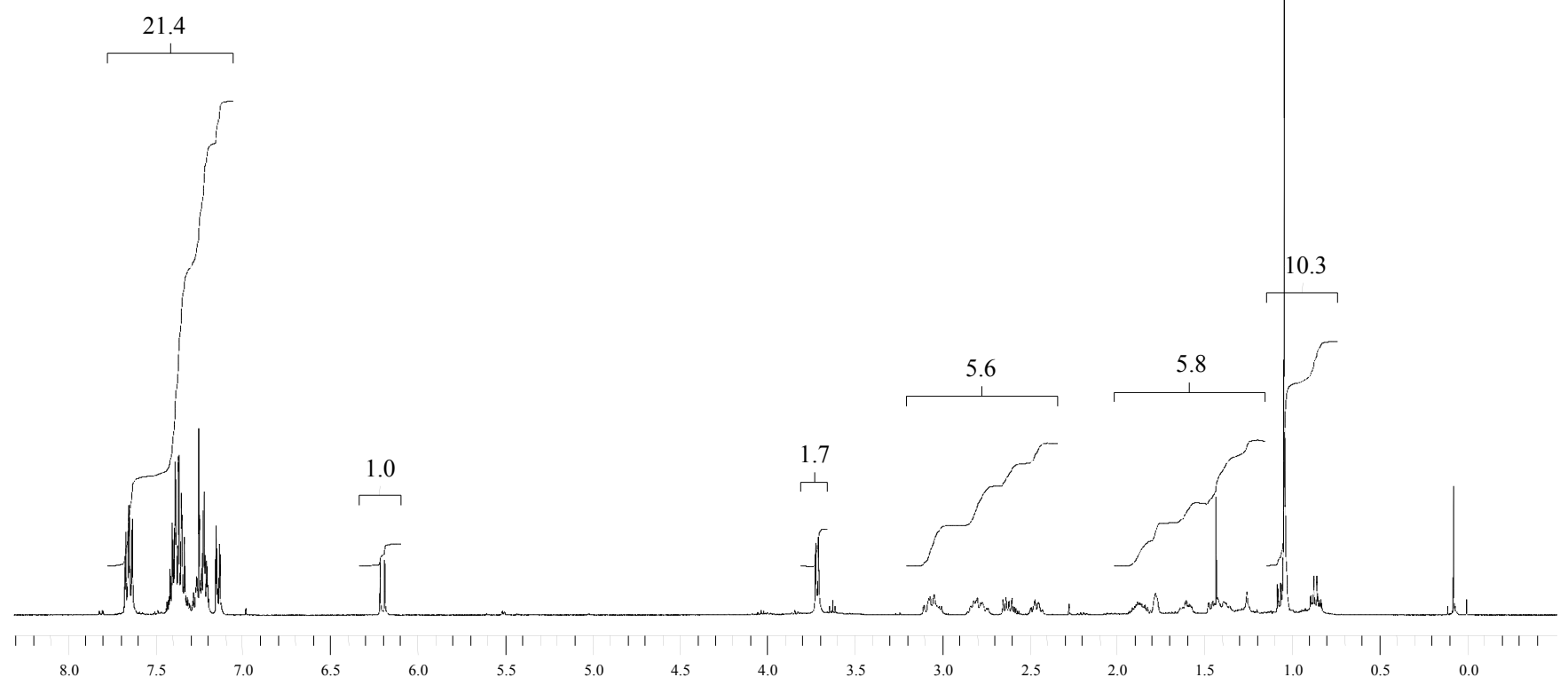




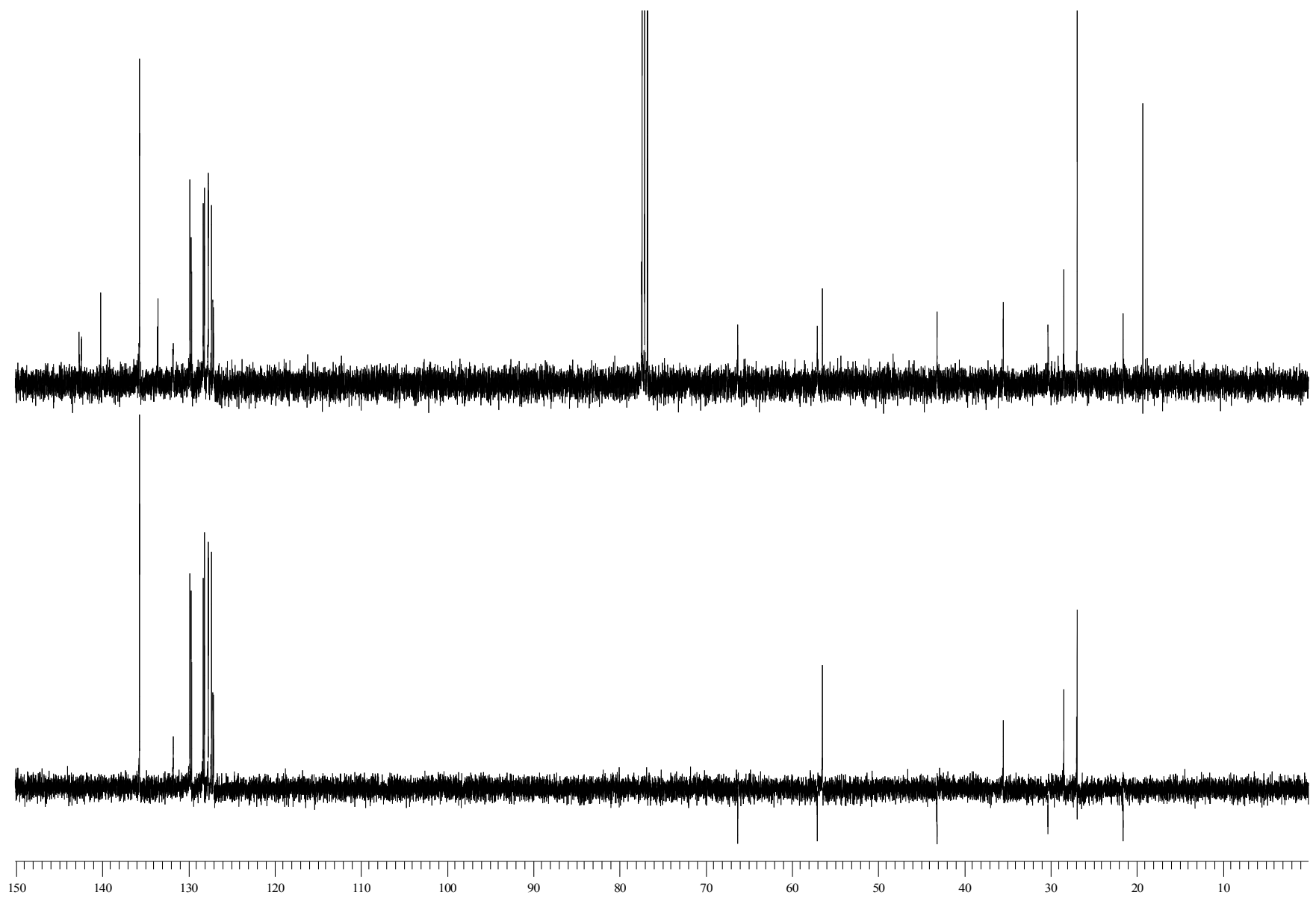

\section{COSY Spectrum}




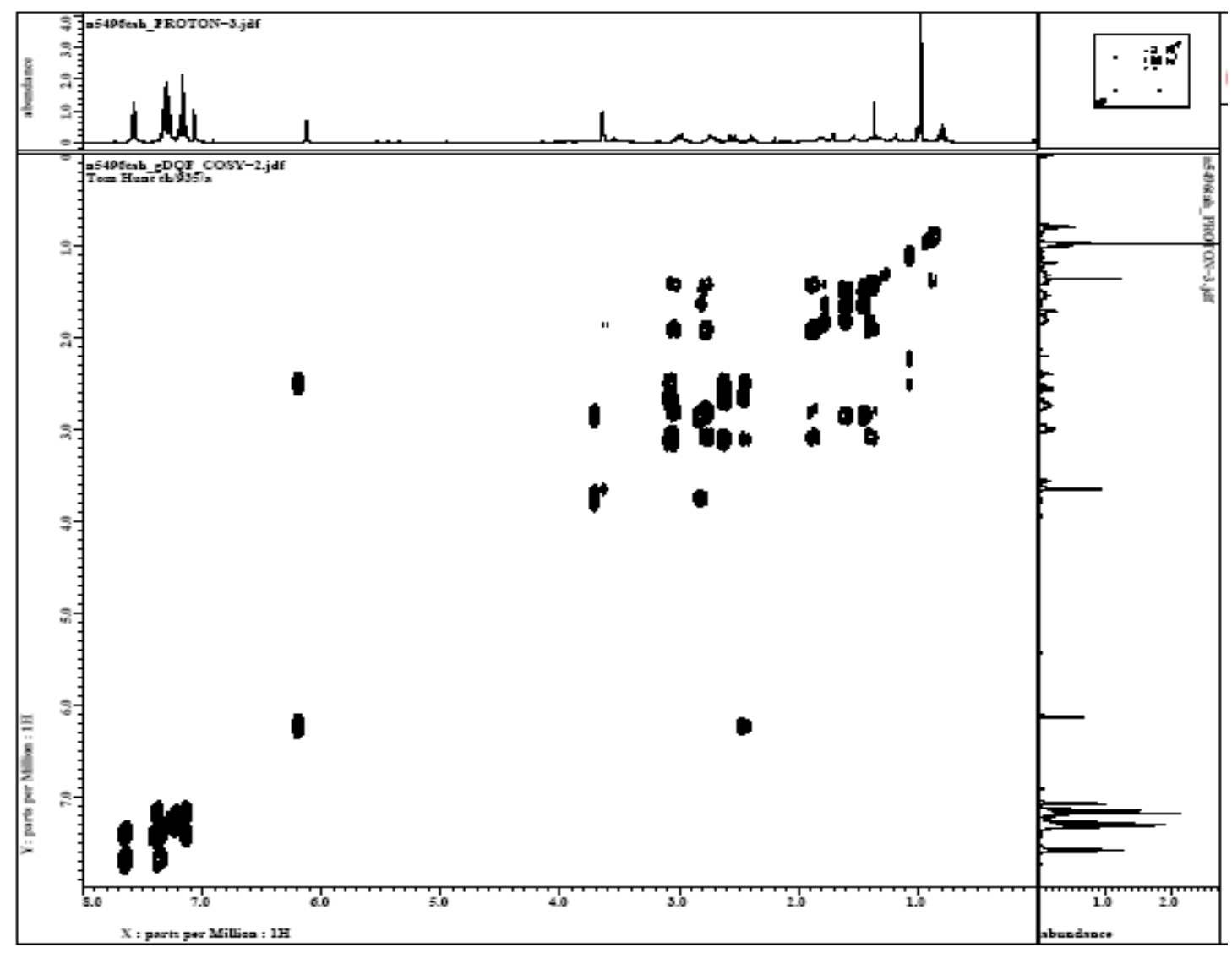

HSQC Spectrum

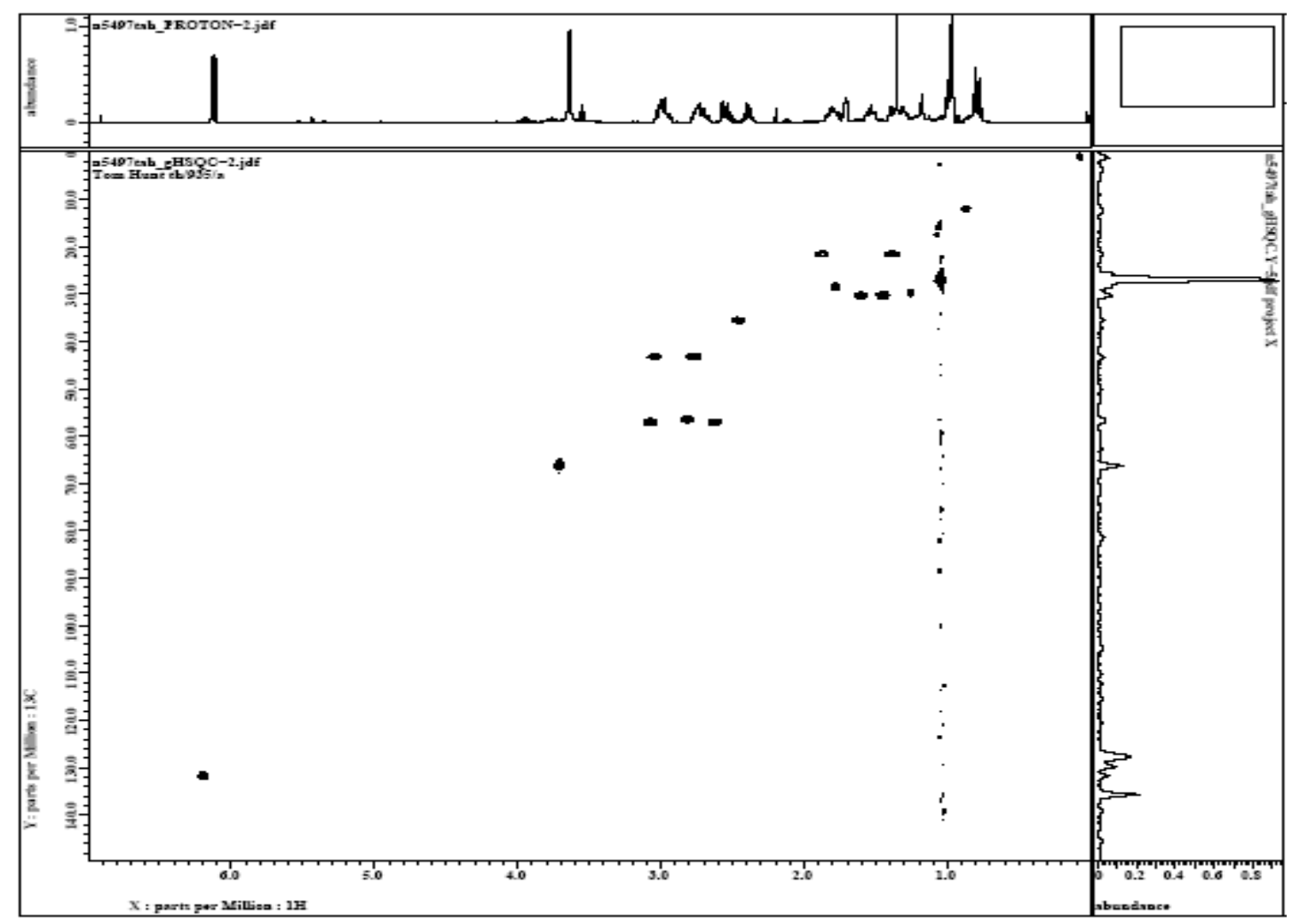

NOESY Spectra 


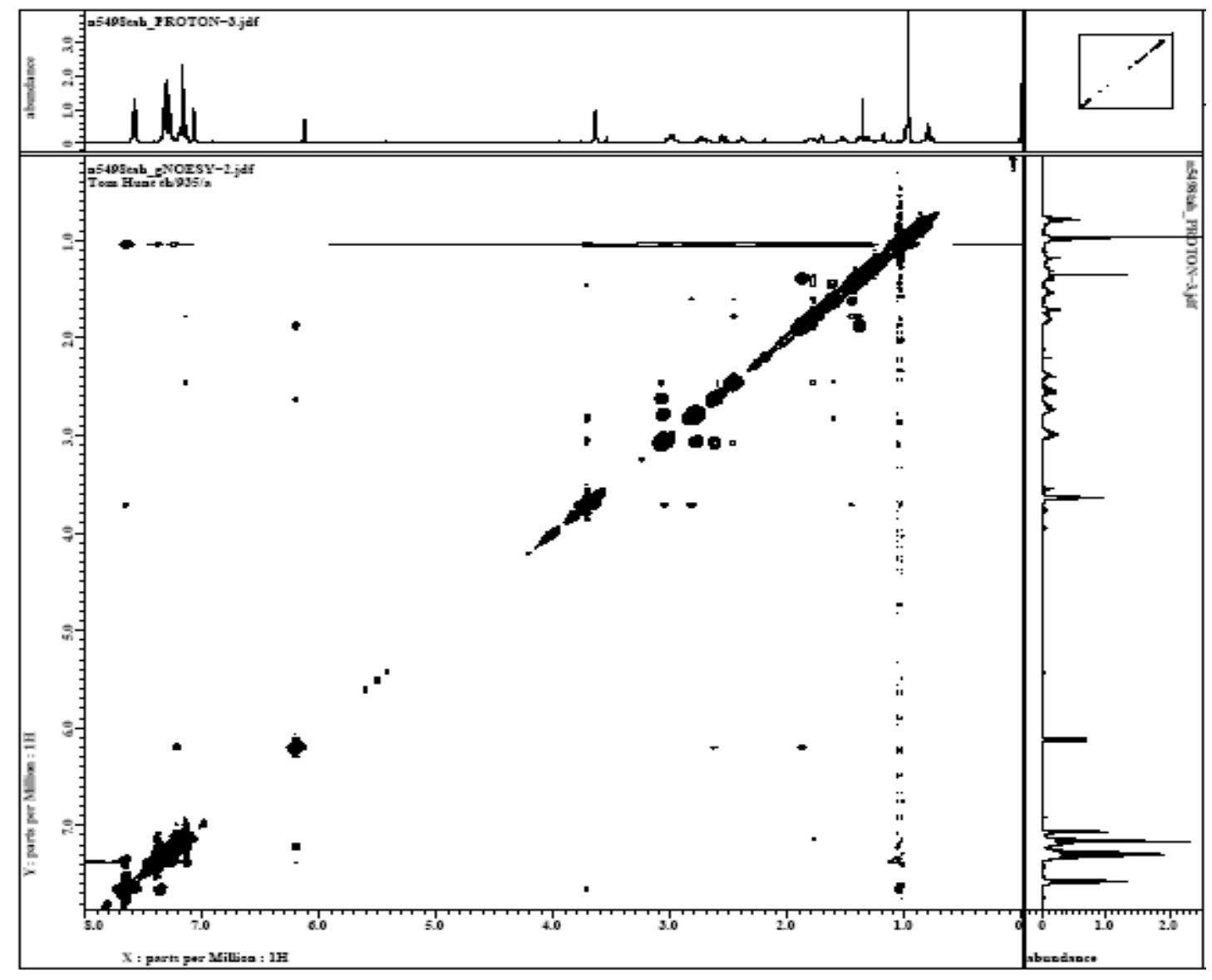




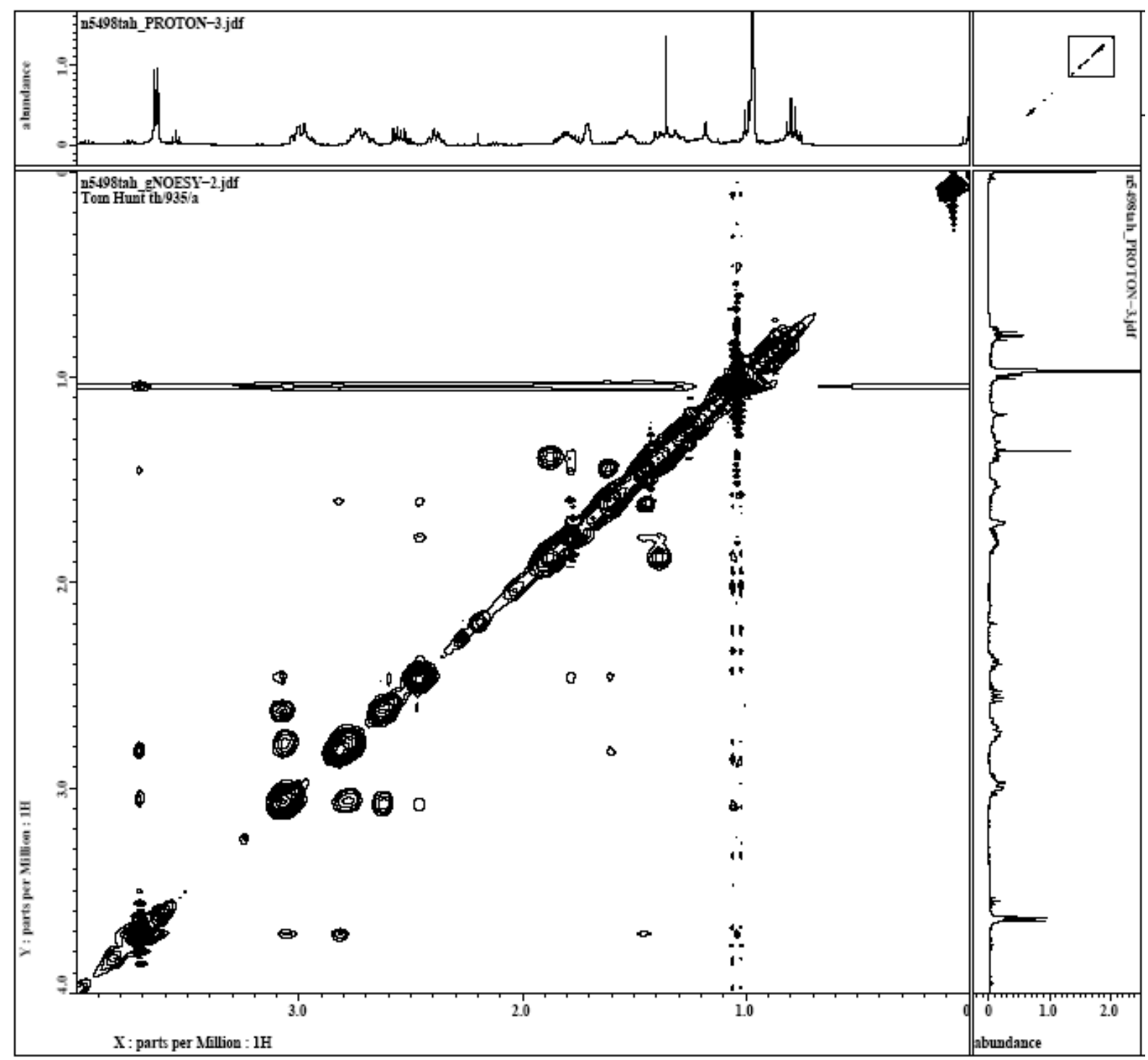

Full NMR assignment of rel-(2S,4R,5S)-2-(\{[tert-Butyl(diphenyl)silyl]oxy $\}$ methyl)-5-(2,2diphenylvinyl)-1-azabicyclo[2.2.2]octane 21b

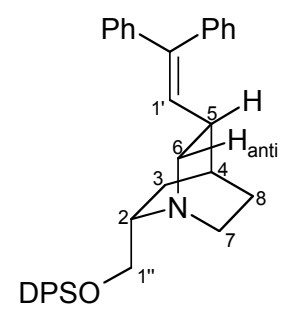

${ }^{1} \mathrm{H}$ NMR (400 MHz, $\mathrm{CDCl}_{3}$ ) $\delta_{\mathrm{H}}:$ 7.71-7.66 (m, 4H, Ar), 7.43-7.21 (m, 14H, Ar), 7.15-7.13 (m, 2H, Ar), $6.18\left(\mathrm{~d}, J=10.0 \mathrm{~Hz}, 1 \mathrm{H}, \mathrm{Ph}_{2} \mathrm{C}=\mathrm{C} \underline{H}\right), 3.79(\mathrm{dd}, J=10.0,5.5 \mathrm{~Hz}, 1 \mathrm{H}, \mathrm{C} \underline{H} \mathrm{HOSi}), 3.73(\mathrm{dd}, J=10.0,7.0$ $\mathrm{Hz}, 1 \mathrm{H}, \mathrm{CH} \underline{H} \mathrm{OSi}) 3.08\left(\mathrm{dd}, J=13.5,10.0 \mathrm{~Hz}, 1 \mathrm{H}, \mathrm{H}-6_{\text {anti }}\right) 3.07-2.91\left(\mathrm{~m}, 2 \mathrm{H}, \mathrm{H}-2\right.$ and $\left.\mathrm{H}-7_{\text {ax }}\right), 2.69$ (dd, $J$ $\left.=13.5,4.5 \mathrm{~Hz}, 1 \mathrm{H}, \mathrm{H}-6_{\mathrm{syn}}\right), 2.58-2.49\left(\mathrm{~m}, 1 \mathrm{H}, \mathrm{H}-7_{\mathrm{eq}}\right), 2.43-2.36(\mathrm{~m}, 1 \mathrm{H}, \mathrm{H}-5), 2.08-2.01\left(\mathrm{~m}, 1 \mathrm{H}, \mathrm{H}-3_{\mathrm{eq}}\right)$, 
1.78-1.74 (m, 1H, H-4), 1.46-1.36 (m, 2H, H-3 ax a H-8 $\left.{ }_{\text {ax }}\right), 1.32-1.22\left(\mathrm{~m}, 1 \mathrm{H}, \mathrm{H}-8_{\text {eq }}\right), 1.07(\mathrm{~s}, 9 \mathrm{H}$, $\left.\operatorname{SiC}\left(\mathrm{C}_{3}\right)_{3}\right) ;{ }^{13} \mathrm{C}$ NMR $\left(100 \mathrm{MHz}, \mathrm{CDCl}_{3}\right) \delta_{\mathrm{C}}: 142.7,141.9$ and $140.1\left(\mathrm{Ph}_{2} \mathrm{C}=\mathrm{CH}\right.$ and $\left.2 \times \underline{C_{\mathrm{ipso}}}, \mathrm{Ar}\right), 135.6$ $\left(4 \times \underline{C} \mathrm{H}_{\text {meta }}, \mathrm{Ph}_{2} \mathrm{Si}, \mathrm{Ar}\right), 133.6$ and $133.5\left(2 \times \underline{C}_{\mathrm{psso}}, \mathrm{Ph}_{2} \mathrm{Si}, \mathrm{Ar}\right), 132.9\left(\mathrm{Ph}_{2} \mathrm{C}=\underline{C} \mathrm{H}\right), 129.8,129.6,128.2$, $128.1,127.6,127.3,127.0,(16 \times \underline{C} \mathrm{H}, \mathrm{Ar}), 66.7\left(\underline{\mathrm{C}} \mathrm{H}_{2} \mathrm{OSi}\right), 57.9\left(\underline{\mathrm{C}} \mathrm{H}_{2}, \mathrm{C}-6\right), 57.6(\underline{\mathrm{C}} \mathrm{H}, \mathrm{C}-2), 42.0\left(\underline{\mathrm{C}} \mathrm{H}_{2}\right.$, C-7), $35.9(\underline{\mathrm{C}} \mathrm{H}, \mathrm{C}-5), 27.9(\underline{\mathrm{C}} \mathrm{H}, \mathrm{C}-4), 27.4\left(\underline{\mathrm{C}} \mathrm{H}_{2}, \mathrm{C}-8\right), 26.9\left(\mathrm{SiC}\left(\underline{\mathrm{C}} \mathrm{H}_{3}\right)_{3}\right), 25.3\left(\underline{\mathrm{C}} \mathrm{H}_{2}, \mathrm{C}-3\right), 19.3$ $\left(\mathrm{SiC}\left(\mathrm{CH}_{3}\right)_{3}\right)$.

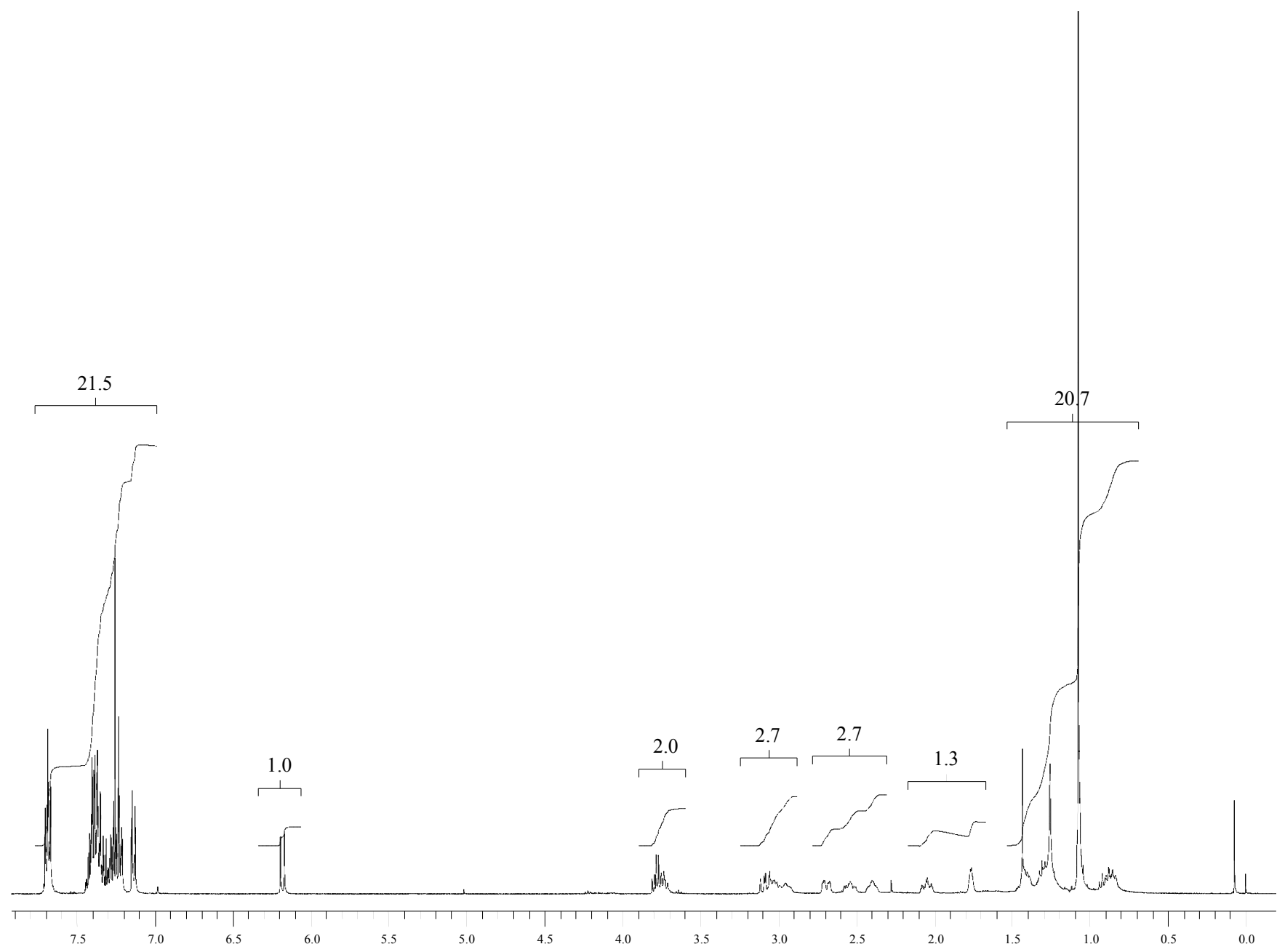



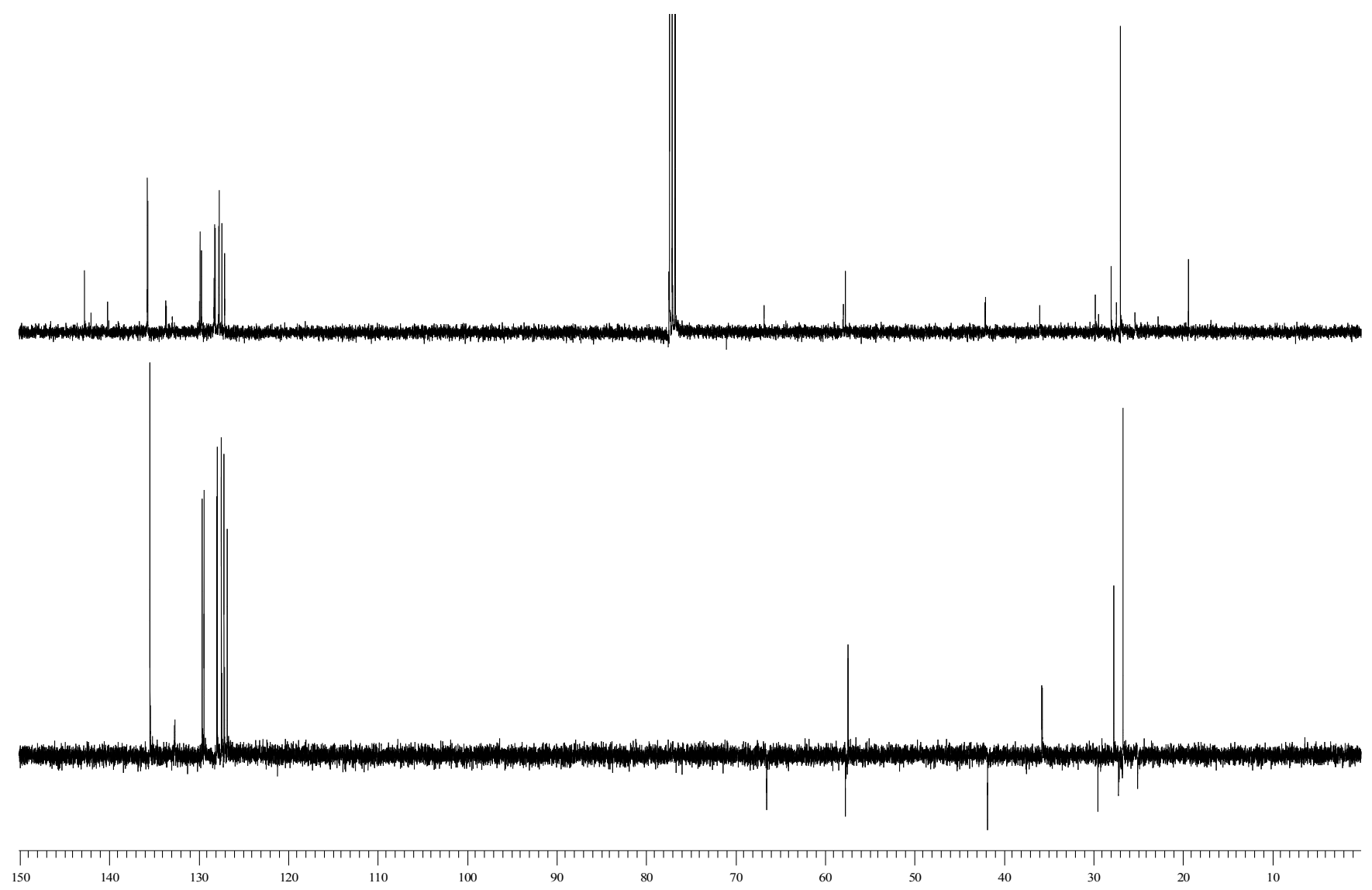

COSY Spectrum 


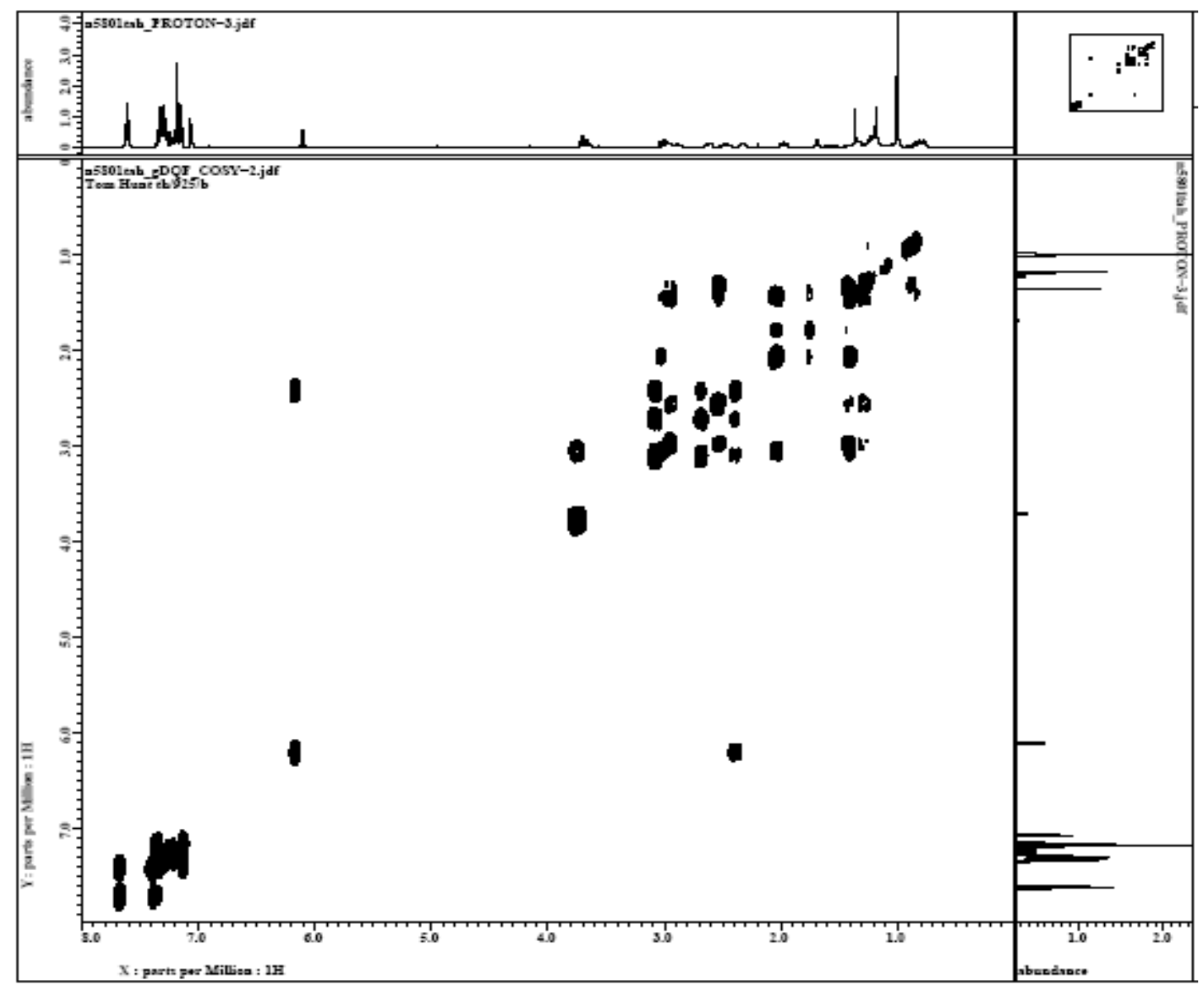

HSQC Spectrum 


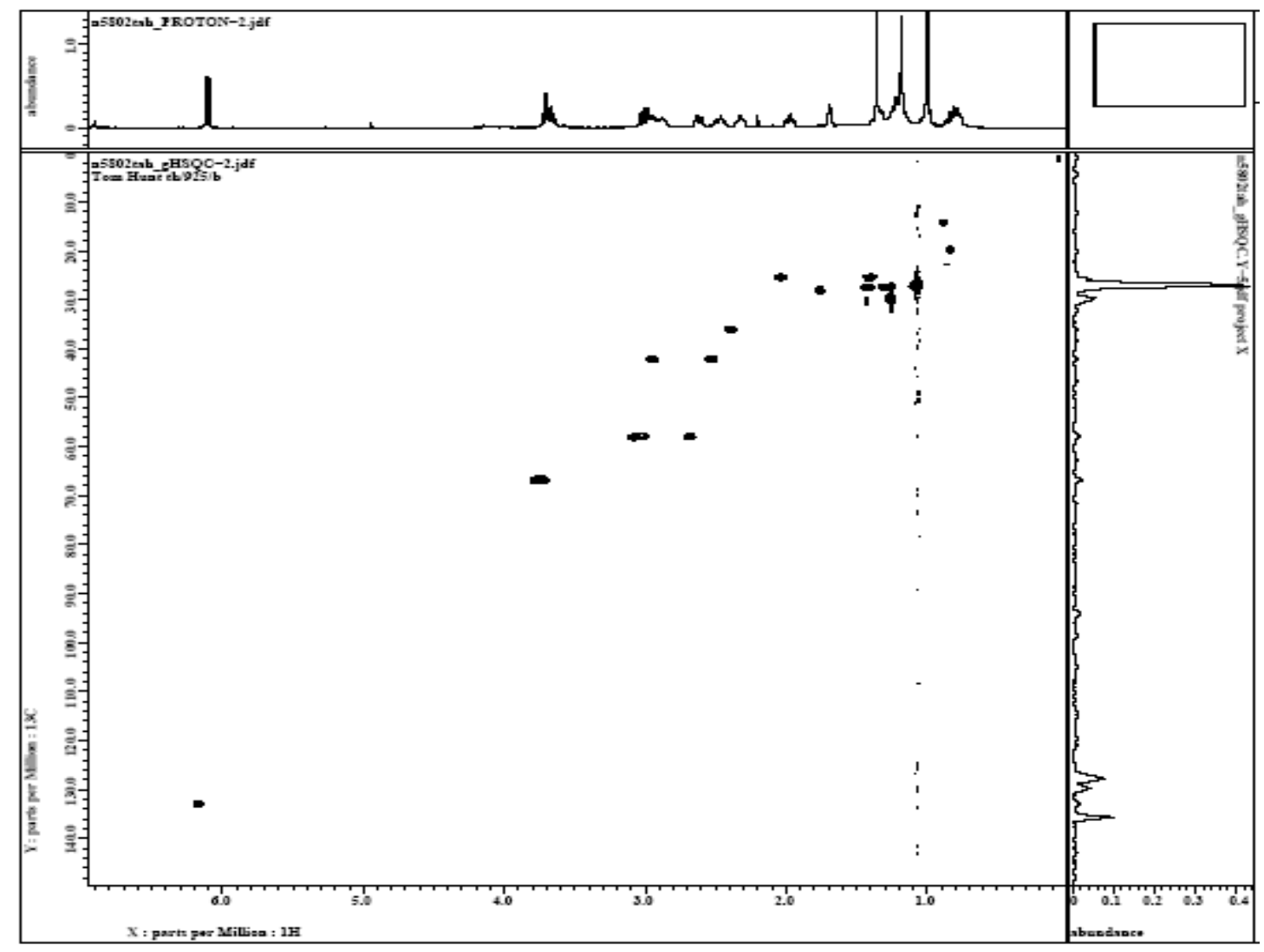

NOESY Spectra

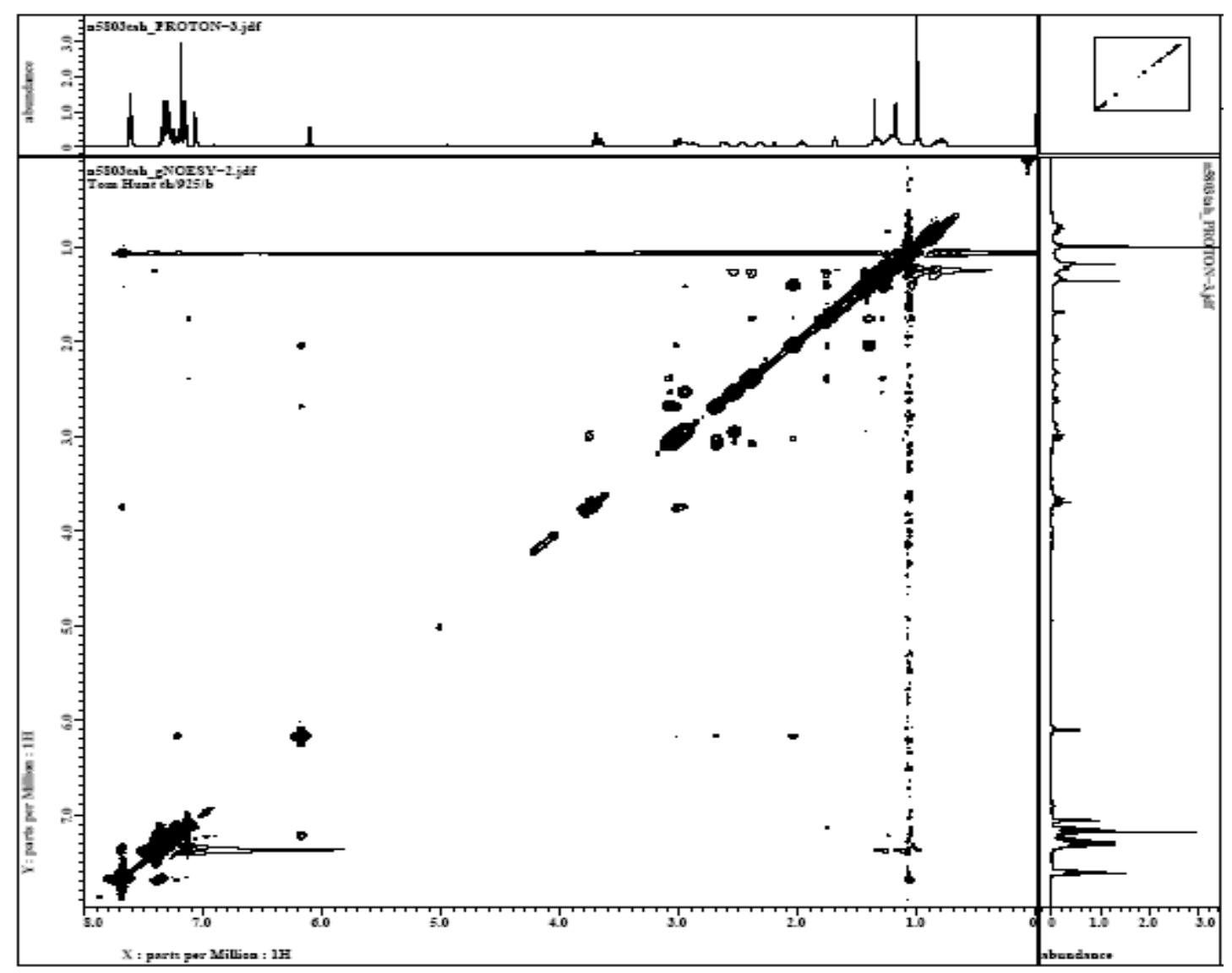




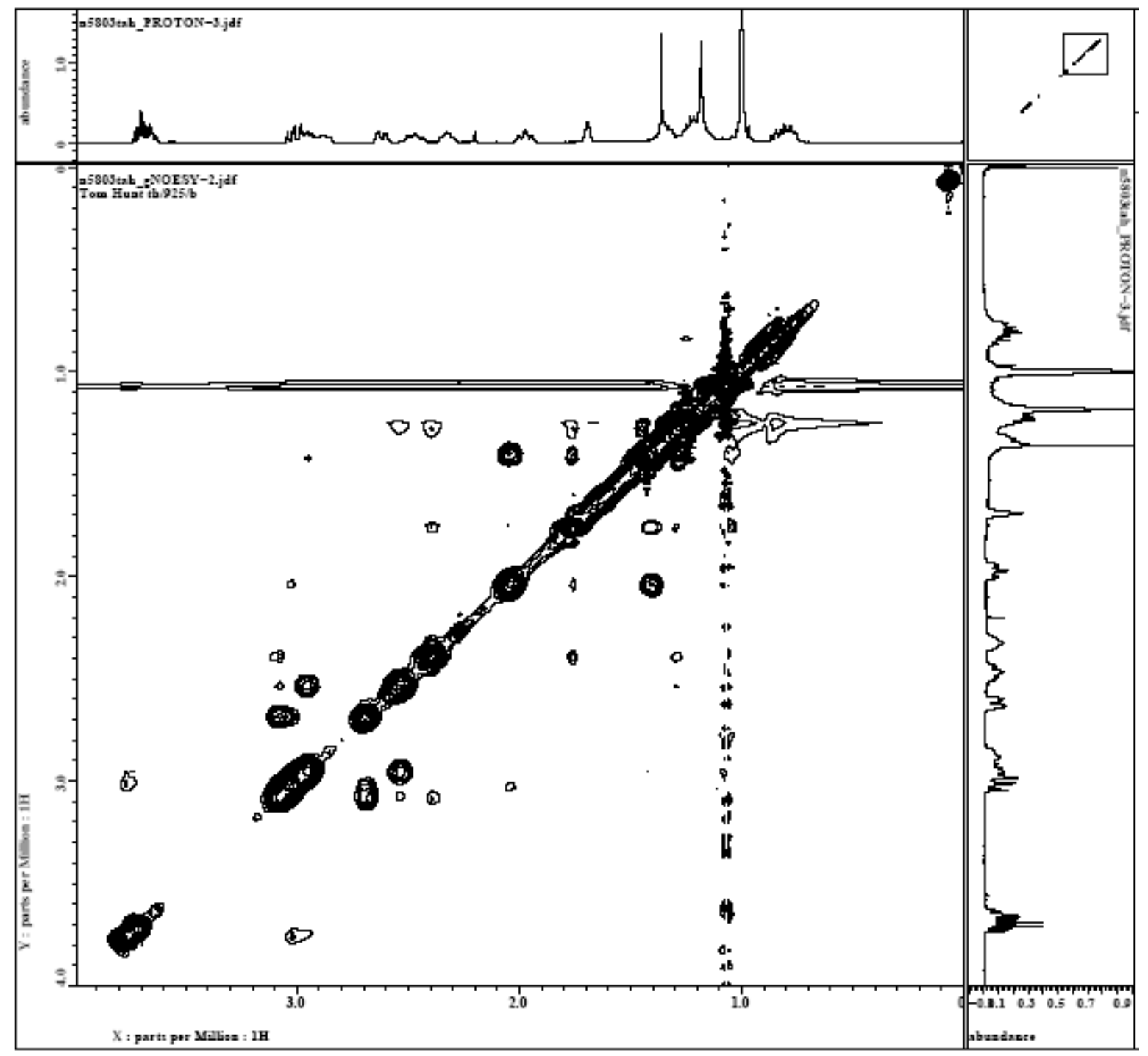

[rel-(2S,4R,5S)-5-(2,2-Diphenylvinyl)-1-azabicyclo[2.2.2]oct-2-yl]methanol 22b

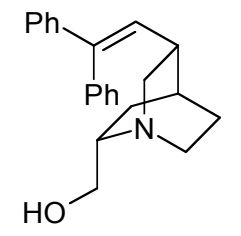

A mixture of silyl ether $\mathbf{2 1 b}(47 \mathrm{mg}, 0.084 \mathrm{mmol})$ and TBAF (0.42 $\mathrm{mL}$ of a $1.0 \mathrm{M}$ solution in THF) in anhydrous THF $\left(10 \mathrm{~mL}\right.$ ) was heated to reflux for $14 \mathrm{~h}$. After cooling to $\mathrm{rt}, \mathrm{NH}_{4} \mathrm{Cl}$ (saturated aqueous solution) was added and this was then extracted with EtOAc. The combined organic phases were dried $\left(\mathrm{MgSO}_{4}\right)$, filtered and evaporated, the crude residue was purified by flash column chromatography (on silica) using $\mathrm{CH}_{2} \mathrm{Cl}_{2}$ as eluent to afford amino alcohol $\mathbf{2 2 b}(19 \mathrm{mg}, 71 \%)$ as a colorless oil. $\mathrm{R}_{\mathrm{f}} 0.30$ 
$\left(\mathrm{CH}_{2} \mathrm{Cl}_{2}: \mathrm{MeOH} 4: 1\right)$; IR: $\left(\mathrm{CH}_{2} \mathrm{Cl}_{2}\right) v_{\max } 3678,3310,2930,2867,1598,1495,1445,1410,1340,1261$, 1199, 1051, 1023, 997, $\mathrm{cm}^{-1} ;{ }^{1} \mathrm{H}$ NMR (400 MHz, $\left.\mathrm{CDCl}_{3}\right) \delta_{\mathrm{H}}: 7.41-7.12(\mathrm{~m}, 10 \mathrm{H}), 6.17(\mathrm{~d}, J=10.5 \mathrm{~Hz}$ 1H), 3.79 (br s, 1H), 3.60-3.54 (m, 2H), 3.31-3.21 (m, 2H), 3.15-3.05 (m, 1H), $2.79(\mathrm{dd}, J=13.5,5.0$ $\mathrm{Hz}, 1 \mathrm{H}), 2.66(\mathrm{ddd}, J=13.5,11.0,5.5 \mathrm{~Hz}, 1 \mathrm{H}), 2.57-2.49(\mathrm{~m}, 1 \mathrm{H}), 2.14-2.06(\mathrm{~m}, 1 \mathrm{H}), 1.84-1.80(\mathrm{~m}$, 1H), 1.56-1.41 (m, 2H), 1.03-0.96 (m, 1H); ${ }^{13} \mathrm{C}$ NMR (100 MHz, $\left.\mathrm{CDCl}_{3}\right) \delta_{\mathrm{C}}: 143.0(\mathrm{C}), 142.2(\mathrm{C}), 139.7$ (C), $131.2(\mathrm{CH}), 129.6(\mathrm{CH}), 128.3(\mathrm{CH}), 128.2(\mathrm{CH}), 127.3(\mathrm{CH}), 127.2(\mathrm{CH}), 62.5\left(\mathrm{CH}_{2}\right), 58.2(\mathrm{CH})$, $56.7\left(\mathrm{CH}_{2}\right), 40.0\left(\mathrm{CH}_{2}\right), 35.6(\mathrm{CH}), 27.3(\mathrm{CH}), 26.8\left(\mathrm{CH}_{2}\right), 24.3\left(\mathrm{CH}_{2}\right) ; \mathrm{LRMS}\left(\mathrm{CI}, \mathrm{NH}_{3}\right) \mathrm{m} / z, 320$ $\left([\mathrm{M}+\mathrm{H}]^{+}, 100 \%\right)$; HRMS $\left(\mathrm{CI}, \mathrm{NH}_{3}\right) \mathrm{m} / z,[\mathrm{M}+\mathrm{H}]^{+}$found 320.2016 ; calcd 320.2014 for $\mathrm{C}_{22} \mathrm{H}_{25} \mathrm{NO}$.

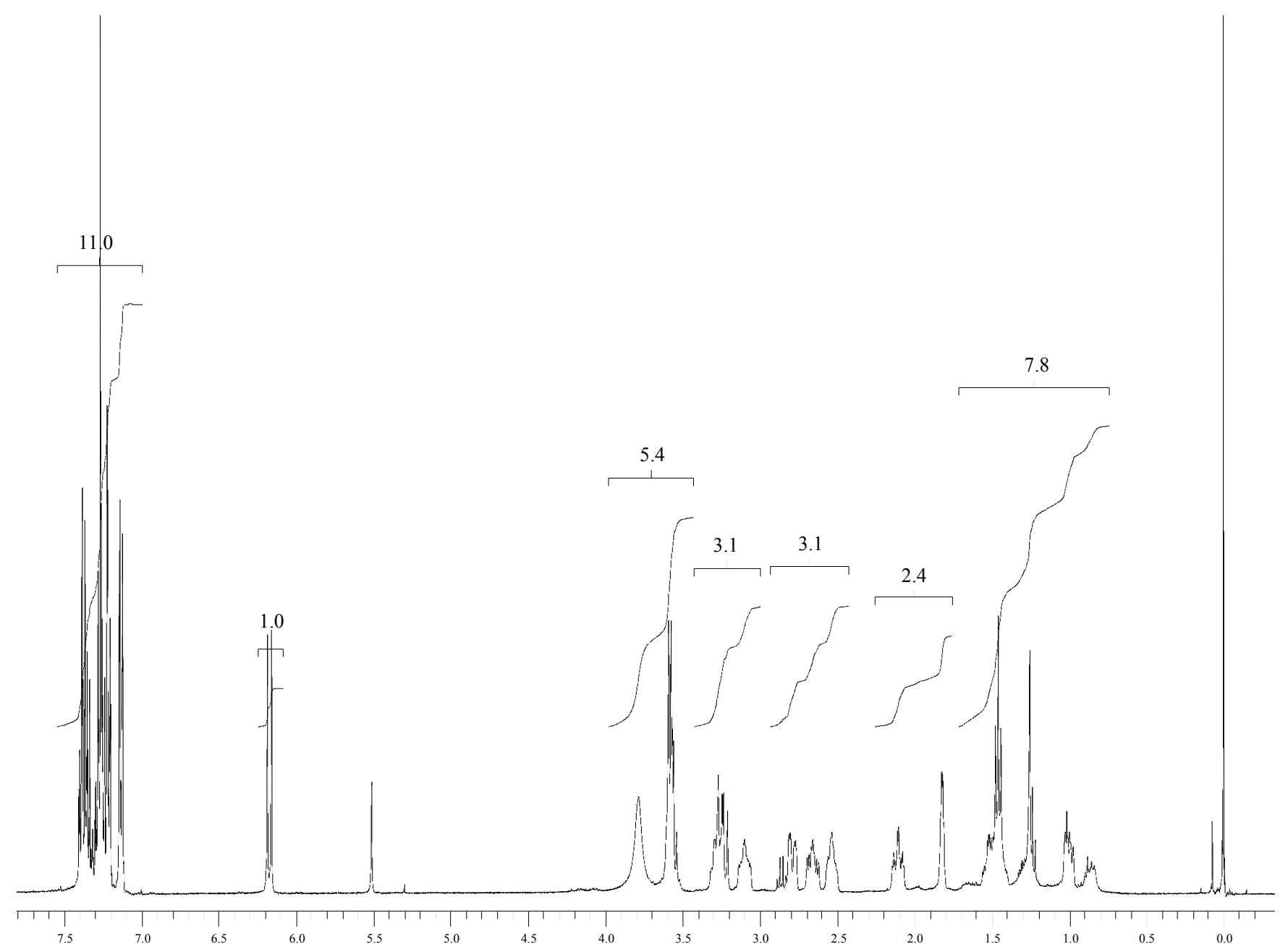



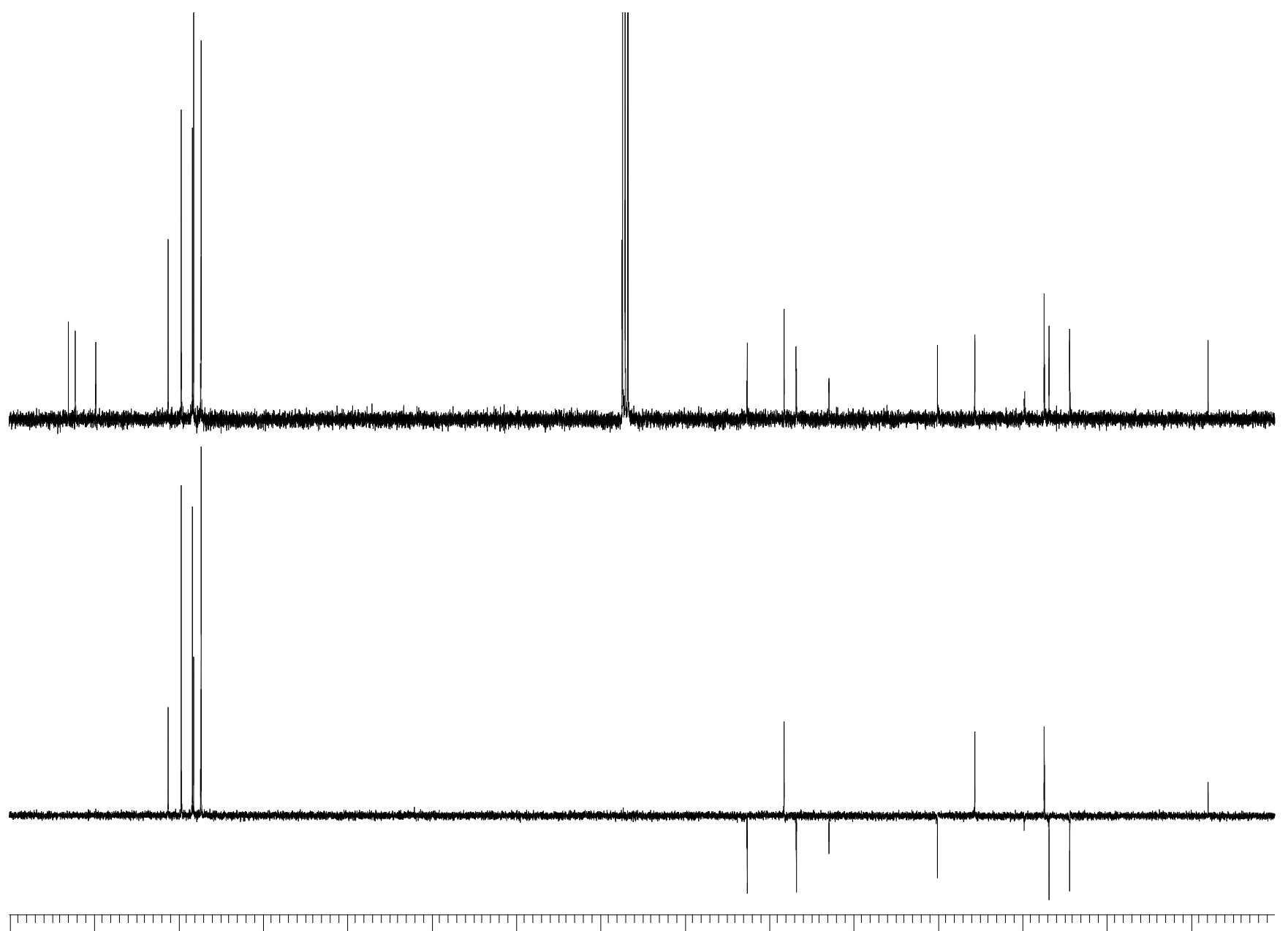
140

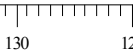

110

COSY Spectrum 


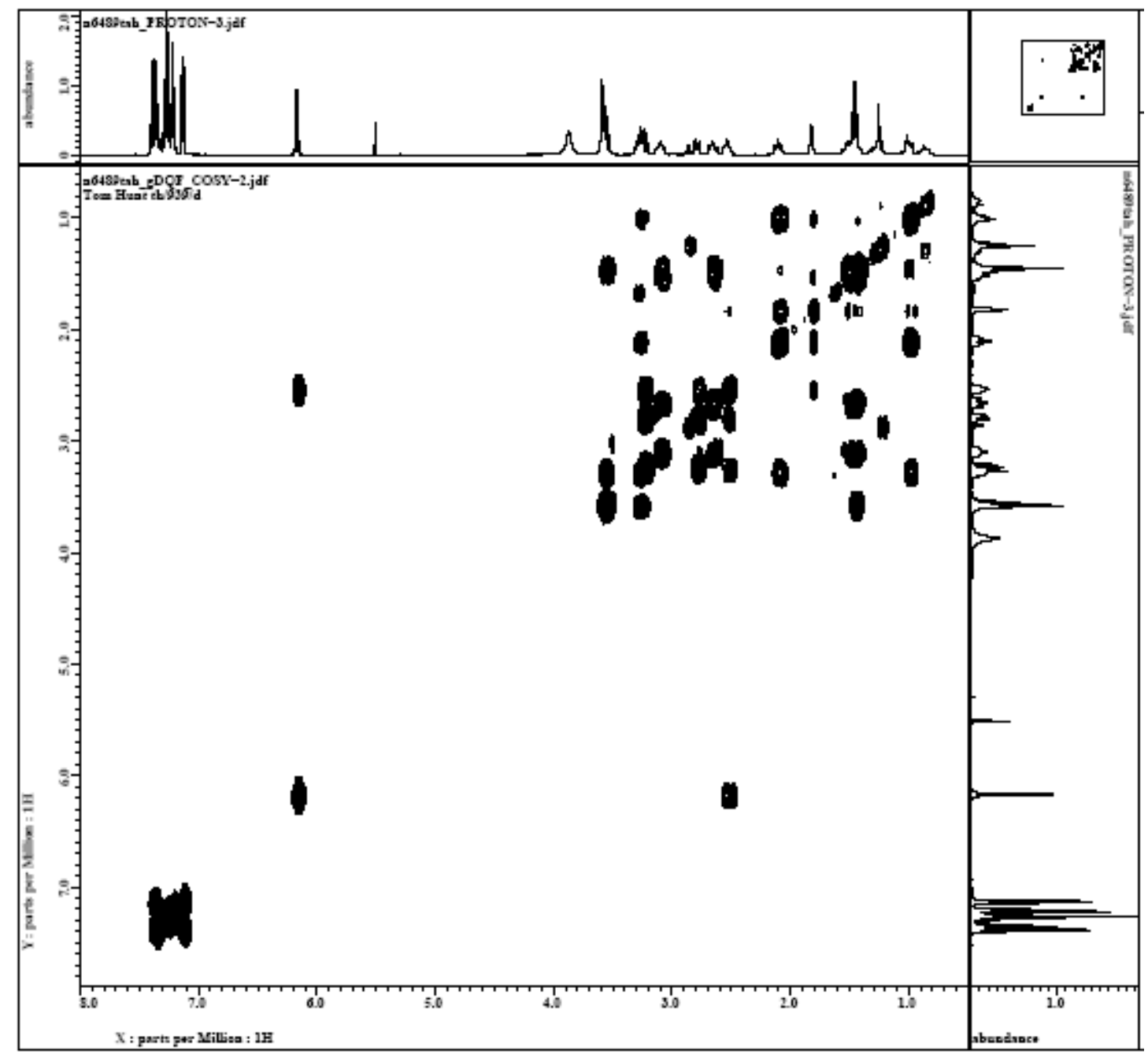

HSQC Spectrum 


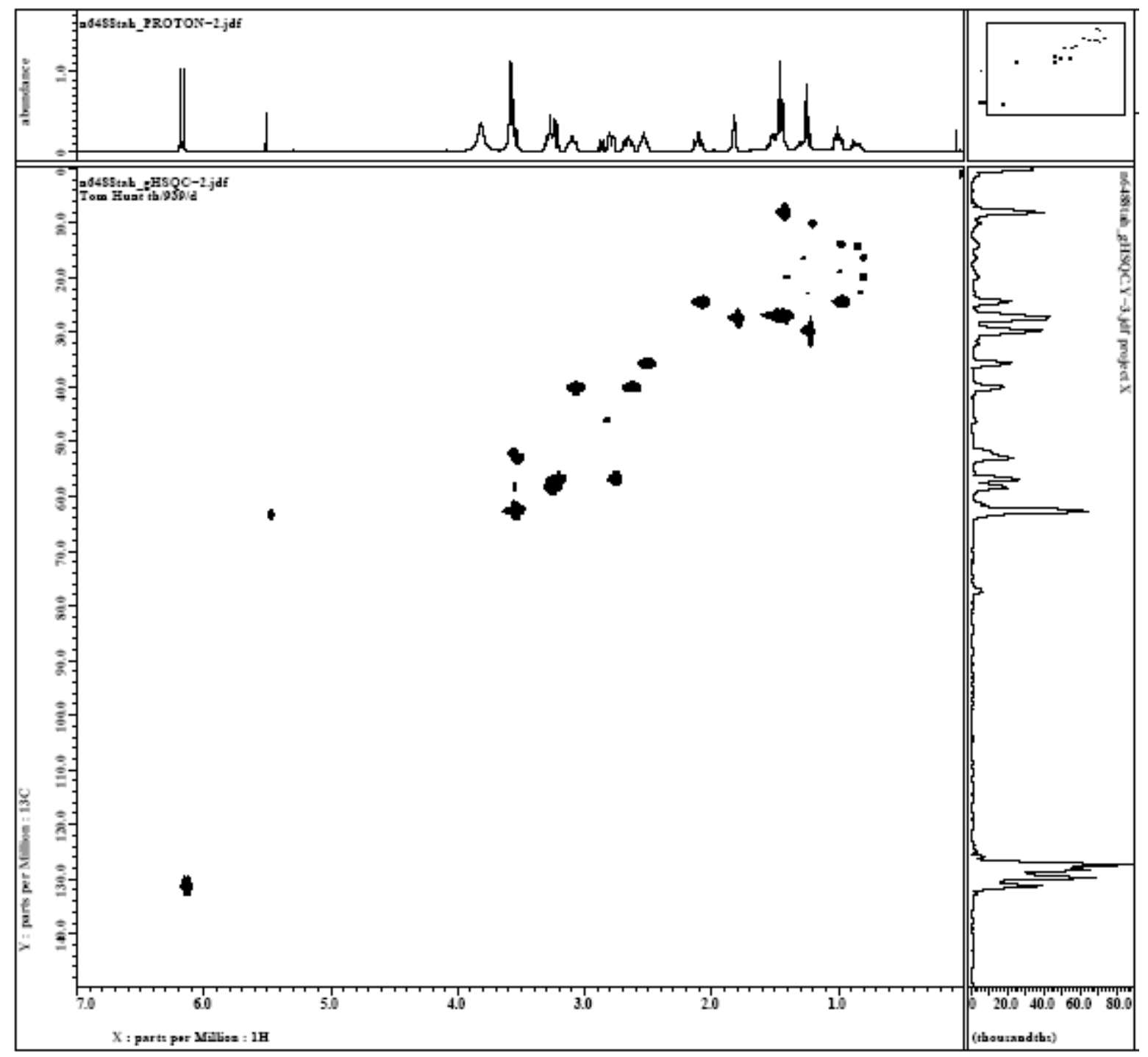

NOESY Spectra 


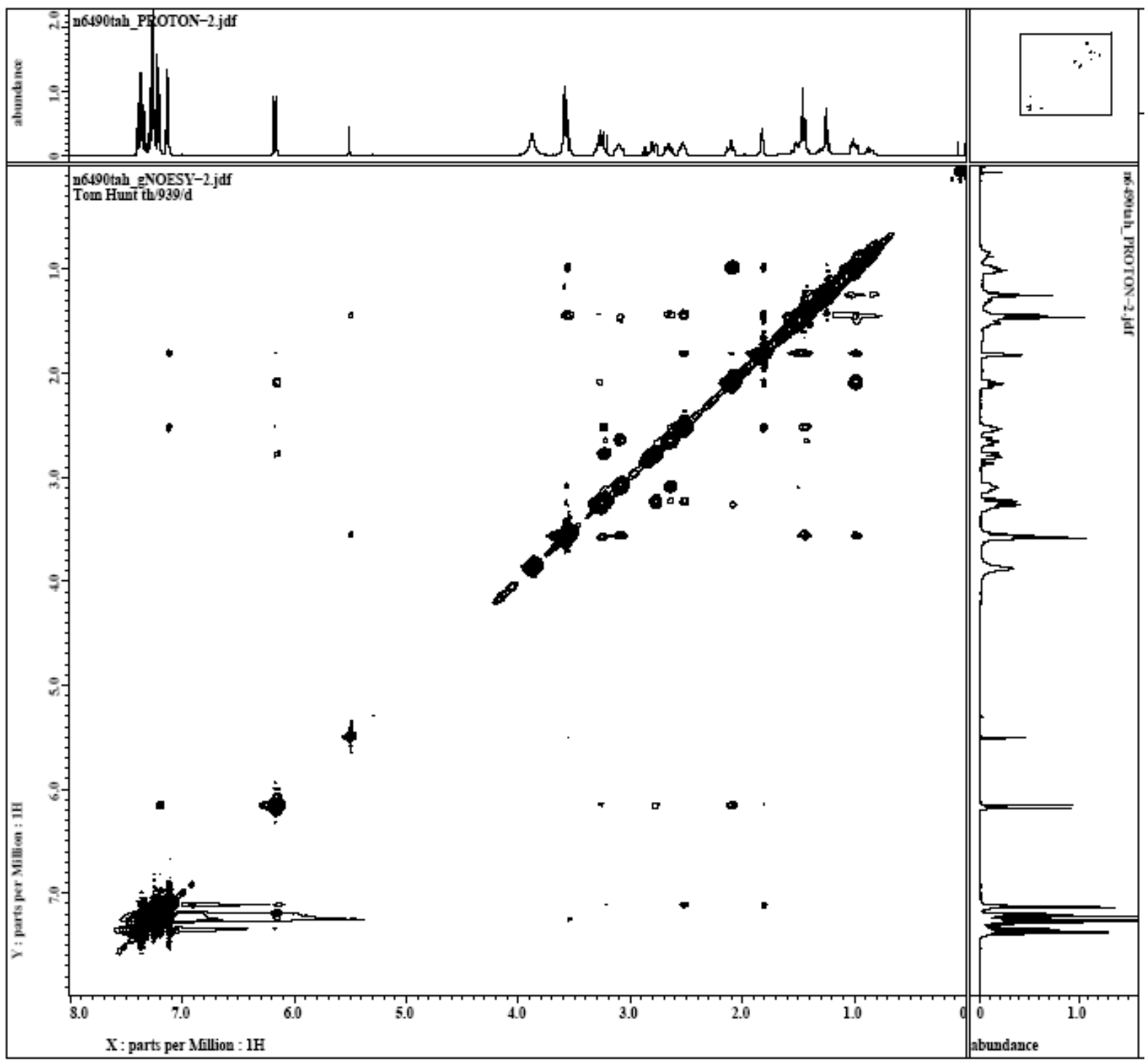




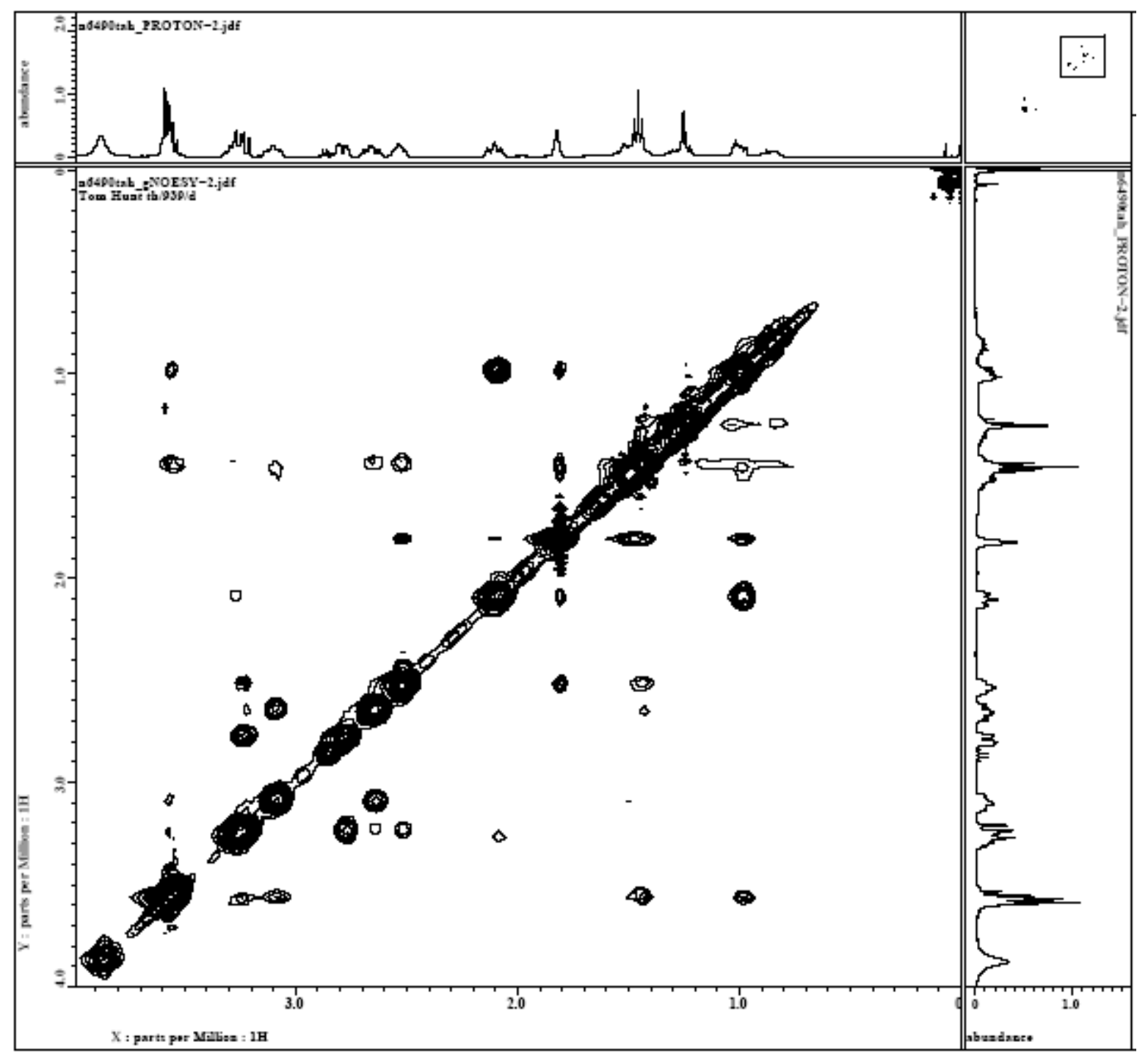

